\title{
AN OVERVIEW OF PERIODIC ELLIPTIC OPERATORS
}

\author{
PETER KUCHMENT
}

Dedicated to the memory of mathematicians and dear friends M. Birman, L. Ehrenpreis, S. Krein, V. Meshkov, and M. Novitskii

Abstract. The article surveys the main topics, techniques, and results of the theory of periodic operators arising in mathematical physics and other areas. Close attention is paid to studying analytic properties of Bloch and Fermi varieties, which significantly influence most spectral features of such operators.

The approaches described are applicable not only to the standard model example of Schrödinger operator with periodic electric potential $-\Delta+V(x)$, but to a wide variety of elliptic periodic equations and systems, equations on graphs, $\bar{\partial}$-operator, and other operators on abelian coverings of compact bases.

Important applications are mentioned. However, due to the size restrictions, they are not dealt with in detail.

\section{Contents}

Introduction

1. $1 D-\mathrm{A}$ brief sketch

1.1. Euler's theorem

1.2. Floquet-Lyapunov theory 346

1.3. Hill operator

2. Lattices in $\mathbb{R}^{n}$

3. Periodic operators

4. Floquet transform and direct integral decomposition

4.1. Floquet transform

4.2. Plancherel and Paley-Wiener type theorems

4.3. Direct integral decomposition

5. Dispersion relation and all that

5.1. Dispersion relation $=$ Bloch variety

5.2. Dispersion relation and the spectrum

5.3. Periodic operators as perturbations of the free ones.

5.4. Born-Karman approximation

5.5. Analytic properties of the dispersion relation $B_{H}$

5.6. Floquet variety

5.7. Nonalgebraicity

Received by the editors October 2, 2015.

2010 Mathematics Subject Classification. Primary 35B27, 35J10, 35J15, 35Q40, 35Q60, 47F05, 58J05, 81Q10; Secondary 32C99, 35C15, 47A53, 58J50, 78M40.

This article contains an extended exposition of the lectures given at Isaac Newton Institute for Mathematical Sciences in January 2015. The work was also partially supported by the NSF grant DMS \# 1517938. The author expresses his gratitude to the INI and NSF for the support. 
5.8. Irreducibility 367

\begin{tabular}{ll|} 
5.9. Extrema & 369 \\
\hline
\end{tabular}

5.10. Dirac cones $\quad 372$

5.11. Fermi surfaces 373

5.12. Bloch bundles 374

5.13. Analyticity in the space of parameters 375

5.14. Inverse problems

6. Spectral structure of periodic elliptic operators 378

6.1. Spectral gaps 378

6.2. Eigenfunction expansion 381

6.3. Absolutely continuous, pure point, and singular continuous parts of the spectrum

6.4. Spectral asymptotics 385

6.5. Wannier functions 386

6.6. Impurity spectra 387

7. Threshold effects 388

7.1. Homogenization 388

7.2. Liouville theorems

7.3. Green's function 389

7.4. Impurity spectra in gaps 389

8. Solutions 389

8.1. Floquet-Bloch expansions 389

8.2. Generalized eigenfunctions and Shnol'-Bloch theorems 390

8.3. Positive solutions 391

8.4. Inhomogeneous equations 391

9. Miscellany 391

9.1. Parabolic time-periodic equations 391

9.2. Semi-crystals 392

9.3. Photonic crystals

9.4. Waveguides 392

9.5. Coverings and noncommutative versions 393

Acknowledgments 393

About the author

References 394

\section{INTRODUCTION}

Elliptic PDEs with periodic coefficients, notably the stationary Schrödinger operator $-\Delta+V(x)$ with a periodic potentia 1$]$, have been intensively studied in physics and mathematics literature for close to a century, due to their crucial role in solid state theory, as well as in other areas (e.g., see the books and surveys [11,30,53, 54, 225, 332, 408, 409 ). In spite of that, some important questions have remained unresolved. The interest in periodic elliptic (and sometimes parabolic) equations and systems received a strong boost in the last decades, due to new applications in areas such as photonic crystals and other metamaterials 245, fluid dynamics [76, 403], carbon nanostructures [218, inverse scattering

\footnotetext{
${ }^{1}$ The potential is usually, but not always, assumed to be real.
} 
method of solving integrable systems [305, 307, and lately topological insulators [28. When the author was preparing his introductory lectures at the Newton Institute in January 2015 [250, he discovered that there was no comprehensive source devoted to the theory of periodic elliptic PDEs and their applications. There are several books and surveys completely or partially devoted to this topic (e.g., 6. 25, 27, 56, 117, 167, 173, 213, 239, 244, 332, 358, 385, 389 ), but none of them collects most of the useful techniques (e.g., the analytic geometry of Bloch and Fermi varieties) and is up to date. Thus, the idea was born to expand the lectures to this survey that would contain the main techniques and results of spectral theory of periodic operators arising in mathematical physics and other areas. These are applicable not only to the standard model example of Schrödinger operator with periodic electric potential $-\Delta+V(x)$, but to a wide variety of elliptic periodic equations and systems, equations on graphs, $\bar{\partial}$-operator, and other operators on abelian coverings of compact bases. Close attention is paid to studying analytic properties of Bloch and Fermi varieties, which significantly influence most properties of such operators.

There are many important applications of what is discussed. However, the author realized at this stage that even a large survey cannot do justice to such applications, nor even to many details of techniques employed. Thus, several sections (especially toward the end of the text) contain mostly hints and pointers to the literature. These choices have been made according to the author's tastes and might not satisfy everyone. The task of a more detailed and comprehensive discussion is postponed until the third iteration of my lectures, which is a monograph in preparation. The same applies to the bibliography of this survey, which is extensive but far from being comprehensive, and many references are given through secondary sources (books and surveys).

\section{1. $1 D$ - A BRIEF SKETCH}

In this section, we survey briefly what is commonly called Floquet (or FloquetLyapunov) theory [153, 273, 274, the main tool in studying periodic linear ODEs and systems of ODEs. Its basics can be found in many ODE textbooks, e.g., in [7, 8, 68, 69. There are also nice books and surveys dedicated to (mostly spectral theory of) the periodic ODEs of the second order, see, e.g., 10, 104, 117, 275, [25, Section 2.8], [356, Ch. 5], [385, Ch. XXI], and [332, Section XIII.16] (see also [105, and the references therein] for the case of singular potentials). We want to attract the reader's attention to the not sufficiently well known amazing treatise [399], which contains an enormous amount of information concerning periodic ODEs and systems of ODEs, including Hamiltonian and canonical systems, parametric resonance, stability domains, various applications, etc. Here, we will only touch upon a few basic things.

1.1. Euler's theorem. Consider a linear system of ODEs

$$
\frac{d x}{d t}=C x, t \in \mathbb{R}, x \in \mathbb{C}^{n},
$$

with a constant $n \times n$ matrix $C$. Then all its solutions look as follows:

$$
x(t)=e^{C t} x_{0},
$$

where $x_{0}$ is the initial value of $x(t)$. One thus obtains the following Euler's theorem [128, which can be found in any ODE textbook: 
Theorem 1.1. All solutions of (1.1) are linear combinations of the exponentialpolynomial solutions of the form

$$
x(t)=e^{i \lambda t} \sum_{j \in \mathbb{Z}, j \geq 0} p_{j} t^{j},
$$

where $i \lambda$ is an eigenvalue of $C$ and the sum is finite.

If $C$ has no Jordan blocks, then only $j=0$ is present in (1.2).

Floquet-Lyapunov theory, sketched below, generalizes this result to the case of systems with periodic coefficients.

\subsection{Floquet-Lyapunov theory. Let us consider a linear system of ODEs}

$$
\frac{d x}{d t}=A(t) x, t \in \mathbb{R}, x \in \mathbb{C}^{n},
$$

with a 1-periodic $n \times n$ matrix function $A(t)$. As promised before, we will not dwell here on imposing weakest conditions on the entries of the matrix [399, Ch. II], assuming that they are continuous. Let $X(t)$ be the $n \times n$ matrix fundamental solution of (1.3). In other words, if

$$
e_{j}=(0, \ldots, 0, \underbrace{1}_{j \text { th entry }}, 0, \ldots, 0)
$$

is the standard basis of $\mathbb{C}^{n}$ and $x_{j}(t)$, the solution of (1.3) such that $x_{j}(0)=e_{j}$, then $X(t)$ has each $x_{j}(t)$ as the $j$ th column. One thinks of $X(t)$ as the operator that shifts by time $t$ along the trajectories. In particular, when the value of $t$ coincides with the period (i.e., is equal to 1 under our assumption), we introduce the following

Definition 1.2. The matrix

$$
M:=X(1)
$$

of the shift by period 1 along the trajectories of the system is said to be the monodromy matrix of equation (1.3).

The main result of the Floquet theory is the following

Theorem 1.3. Floquet theorem. There exists a 1-periodic matrix function $P(t)$ and a constant matrix $C$, such that

$$
X(t)=P(t) e^{C t} .
$$

Remark 1.4. Notice that

$$
M=X(1)=e^{C} .
$$

Corollary 1.5. Any solution of (1.3) is a linear combination of Floquet (or Floquet-Bloch) solutions

$$
x(t)=e^{i k t} \sum_{j \in \mathbb{Z}^{+}} p_{j}(t) t^{j},
$$

where the sum is finite, coefficients $p_{j}(t)$ are 1-periodic, and $e^{i k}$ are the eigenvalues of the monodromy matrix $M$.

When the monodromy matrix does not have Jordan blocks, only the term with $j=0$ is present. In this case, the solution is $x(t)=e^{i k t} p(t)$ with a 1-periodic function $p(t)$ and is sometimes called a Bloch solution. 
Definition 1.6. The eigenvalues $z:=e^{i k}$ of the monodromy matrix $M$ are called Floquet multipliers and numbers $k$ are called quasimomenta or crystal momenta (the latter names coming from solid state physics [11]).

Remark 1.7. One notices that the value of a quasimomentum $k$ is defined only modulo $2 \pi \mathbb{Z}$-shifts 2

In fact, the following, stronger than Theorem 1.3. result holds:

Theorem 1.8. Lyapunov reduction theorem. There exists a periodic invertible matrix-function $B(t)$ such that the substitution $x(t)=B(t) y(t)$ reduces the system (1.3) to the one with a constant matrix $C$ :

$$
\frac{d y}{d t}=C y(t)
$$

1.3. Hill operator. Studying higher-order ODEs with periodic coefficients can be reduced to the case of first-order systems. It is, however, useful to consider the special case of the so called Hill operator:

$$
H=-\frac{d^{2}}{d x^{2}}+V(x)
$$

with a "nice" real 1-periodic potential $V$ on $\mathbb{R}$. Again, we will assume the excessive condition of continuity of $V$, although much weaker conditions suffice (see, e.g., 104, 117, 275, 332, 399]). The domain of the operator will be, by definition, the Sobolev space $H^{2}(\mathbb{R})$. Defined this way, the operator is self-adjoint 3 [117].

1.3.1. Monodromy, discriminant, and such. We will be interested in the spectral problem for the Hill operator:

$$
-\frac{d^{2} u}{d x^{2}}+V(x) u=\lambda u
$$

In accordance with the general Floquet theory approach, we consider the fundamental system of two (analytic in $\lambda$ ) solutions $\phi(x, \lambda), \psi(x, \lambda)$ :

$$
\phi(0, \lambda)=\psi_{x}^{\prime}(0, \lambda)=1, \phi_{x}^{\prime}(0, \lambda)=\psi(0, \lambda)=0 .
$$

This enables us to consider the monodromy matrix

$$
M(\lambda):=\left(\begin{array}{cc}
\phi(1, \lambda) & \psi(1, \lambda) \\
\phi_{x}^{\prime}(1, \lambda) & \psi_{x}^{\prime}(1, \lambda)
\end{array}\right)
$$

The determinant $\operatorname{det} M(\lambda)$ is the Wronskian of this fundamental system of solutions, evaluated at $t=1$. Since the Wronskian is constant in time and equal to 1 at $t=0$, we conclude that

$$
\operatorname{det} M(\lambda)=1 .
$$

Thus, since we are interested in the eigenvalues of the monodromy matrix, all the pertinent information is contained in the trace of $M$ :

$$
\Delta(\lambda):=\operatorname{Tr} M(\lambda)=\phi(1, \lambda)+\psi_{x}^{\prime}(1, \lambda),
$$

which is called the discriminant or the Lyapunov function of the Hill operator (1.8).

\footnotetext{
${ }^{2}$ See the discussion of dual lattices and quasimomenta in Section 2

${ }^{3}$ It is not hard to show that if one defines the operator on the space $C_{0}^{\infty}(\mathbb{R})$, it will be essentially self-adjoint, with the only self-adjoint extension described above.
} 
Thus, all Floquet multipliers $z$, being the eigenvalues of $M$, can be found from the secular equation

$$
z^{2}-\Delta(\lambda) z+1=0
$$

Its roots provide Floquet multipliers and quasimomenta for a given $\lambda$ :

$$
e^{i k}=z=0.5\left(\Delta(\lambda) \pm \sqrt{\Delta(\lambda)^{2}-4}\right) .
$$

It is now easy to come up with the following result (a compilation of various statements from standard books, e.g., [117, 399]):

\section{Theorem 1.9.}

(1) If $|\Delta(\lambda)|<2$, then

- the Floquet multipliers are complex, distinct, of the absolute value 1, and complex conjugate to each other.

- In particular, the quasimomentum is real and if $k$ is a quasimomentum, then $-k$ is one 4

- All solutions of $H u=\lambda u$ are bounded.

- The Lyapunov exponent5 is equal to zero.

(2) If $|\Delta(\lambda)|=2$, then

- The two Floquet multipliers coincide and are equal to 1 or -1 .

- Quasimomentum is either $k=0$ or $k=\pi(\bmod 2 \pi)$.

- All solutions of $H u=\lambda u$ are bounded or polynomially bounded.

- The Lyapunov exponent is equal to zero.

(3) If $|\Delta(\lambda)|>2$, then

- The Floquet multipliers are real, distinct, and reciprocal to each other.

- Quasimomenta are complex.

- Solutions of $H u=\lambda u$ grow exponentially.

- The Lyapunov exponent is positive and equal to $|\Im k|$.

Let us look at the case of the free operator (i.e., with zero potential). A straightforward calculation shows then that the free discriminant is $\Delta_{0}(\lambda)=2 \cos (\sqrt{\lambda})$. In particular, it is an entire function of exponential order $1 / 2$, i.e.,

$$
\left|\Delta_{0}(\lambda)\right| \leq C e^{C|\lambda|^{1 / 2}}
$$

for some $C>0$. Its graph looks as shown in Figure 1. The properties of the discriminant in presence of a periodic potential have also been studied thoroughly (see, e.g., [117, 275, 385]). As in the free case, it is an entire function of exponential order $1 / 2$ :

Figure 2 gives an idea of its behavior.

$$
|\Delta(\lambda)| \leq C e^{C|\lambda|^{1 / 2}}
$$

Notice that the extrema of the free discriminant are located exactly on the boundary of the horizontal strip $|\Delta| \leq 2$. They get shifted in the periodic case (without any new ones being created) but cannot move into the interior of this strip (see detailed considerations, e.g., in [117]). In particular, the pieces of the function

\footnotetext{
${ }^{4}$ This observation also follows from the fact that, due to the potential being real and for real $\lambda$, the complex conjugate of a solution is a solution as well. In physics terms, this is a reflection of the time reversibility of the dynamical system with the Hill operator as the Hamiltonian.

${ }^{5}$ The Lyapunov exponent $r$ characterizes the maximal possible rate $e^{r t}$ of the exponential growth of solutions.
} 


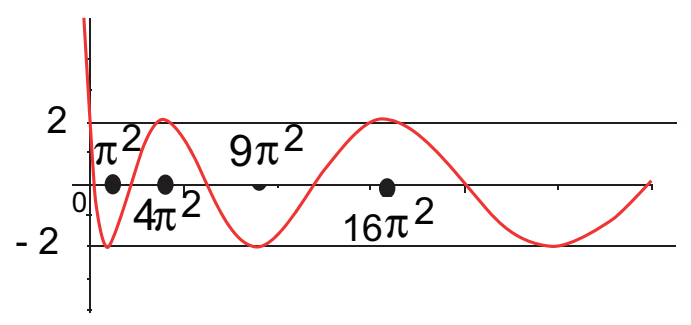

FiguRE 1. The graph of the free discriminant.

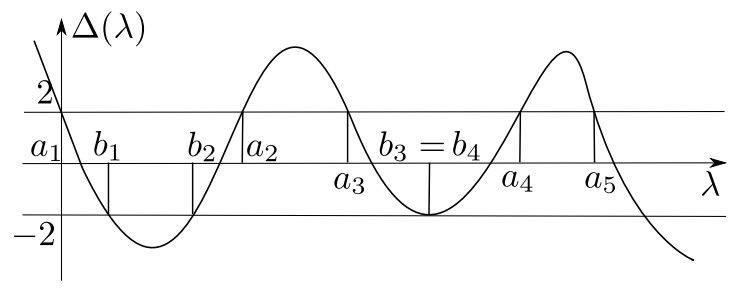

Figure 2. Discriminant for a periodic Hill equation.

$\Delta(\lambda)$ between its consecutive hits of the lines $\Delta= \pm 2$, are monotonic. We will provide a simple explanation of this monotonicity a little bit later. The reader will see later on that absence of any analog of this monotonicity in higher dimensions is responsible for significant changes in some spectral properties in comparison with $1 D$.

Definition 1.10. The closures of the segments where $|\Delta(\lambda)|<2$ (shown in red in Figure 3) are called stability zones or spectral bands. The segments between the bands are called instability zones or spectral gaps. We will denote by $g_{n} \geq 0$ the length of the $n$th gap, $n=1,2, \ldots$

Remark 1.11. Thus, we conclude that the spectral bands are characterized by the quasimomentum being real and Lyapunov exponent being equal to zero. Correspondingly, the spectral gaps are characterized by the complex quasimomentum and positive Lyapunov exponent.

1.3.2. Spectrum of the Hill operator. So far, the name "spectral band" was not explicitly related to the spectrum of the Hill operator $H=-d^{2} / d x^{2}+V(x)$. It is not hard to show (which will be explained later on in the higher dimensions) that the spectrum $\sigma(H)$ indeed coincides with the union of all spectral bands. E.g., existence of bounded generalized solutions inside the spectral bands allows one to show that the bands do belong to the spectrum. Indeed, cutting off these solutions at infinity provides approximate eigenfunctions. On the other hand, one can show that inside the gaps there is no spectrum.

We now collect some important features of the spectrum:

\section{Theorem 1.12.}

(1) There exists a sequence of real numbers

$$
a_{1}<b_{1} \leq b_{2}<a_{2} \leq a_{3}<\cdots \mapsto \infty
$$




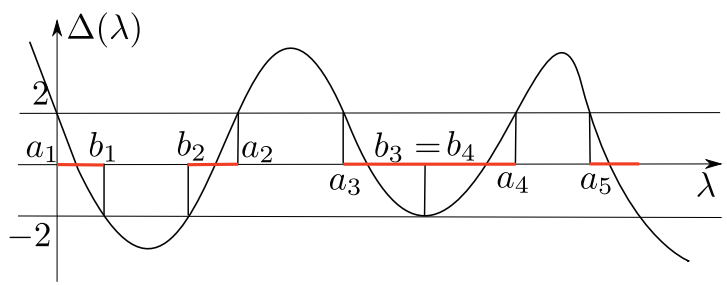

Figure 3. Stability zones (spectral bands), shown in red, are separated by the instability zones (spectral gaps)

(ends of the spectral bands) such that the spectrum $\sigma(H)$ coincides with the union of all spectral bands $I_{j}$

$$
\sigma(H)=\bigcup_{j \in \mathbb{Z}^{+}} I_{j}
$$

where

$$
I_{1}=\left[a_{1}, b_{1}\right], I_{2}=\left[b_{2}, a_{2}\right], I_{3}=\left[a_{3}, b_{3}\right], \ldots
$$

(2) The spectral bands have finite lengths and do not overlap but might touch (see the proof in Lemma 1.17).

(3) Generically (in the Baire category sense), w.r.t. to the $C^{\infty}$ potential, all gaps are present (open), i.e., there are no equalities in (1.16), so the bands do not touch 354 .

(4) Finite gap (finite zone) potentials, i.e., those that lead to finitely many open gaps only, are very special and can be all described [112,113, 306, 307. However, they form a dense set in the space of all $C^{\infty}$ potentials [72,277, 278 .

(5) There are no open gaps if and only if the potential is constant (the famous Borg's theorem [4]).

(6) The rate of the gaps' sizes $g_{n}$ decay when $n \rightarrow \infty$ determines smoothness of the potential [103 105, 276]. For instance, $C^{\infty}$ potentials correspond to the gap size decaying faster than any power of $1 / n$. See, e.g., [105, pp. 96-97] for detailed history.

(7) There are isospectral potentials. The sets of isospectral finite-zone potentials form tori [282, 283, 306].

1.3.3. Dispersion relation. We will introduce now the important notion of the dispersion relation for the Hill operator. It is rarely discussed in mathematics literature devoted to periodic ODEs, but it is central in solid state physics and crucial for the higher-dimension considerations (see [151,152 for the Riemann surface of quasimomentum techniques in 1D).

The dispersion relation describes the spectral parameter $\lambda$ as a (multiple valued) function of the crystal momentum $k$ :

Definition 1.13. The dispersion relation (or the Bloch variety $B_{H}$ ) of the Hill operator $H$ is the subset of $\mathbb{R}_{k} \times \mathbb{R}_{\lambda}$ defined as follows:

$$
B_{H}:=\left\{(k, \lambda) \in \mathbb{R}^{2} \mid H u=\lambda u\right. \text { has a nontrivial Floquet-Bloch }
$$
solution $u(x)=e^{i k x} p(x)$ with the quasimomentum $\left.k\right\}$. 
The complex dispersion relation (or complex Bloch variety) $B_{H, \mathbb{C}}$ is defined analogously, only allowing both $k$ and $\lambda$ to be complex.

One immediately obtains the following statement:

\section{Theorem 1.14.}

(1) The dispersion relation is $2 \pi$-periodic with respect to $k$.

(2) The real dispersion relation is even with respect to $k$. The complex one is invariant with respect to the mapping $(k, \lambda) \mapsto(-k, \bar{\lambda})$. (Indeed, due to the potential being real, the complex conjugate of a Floquet solution is also a Floquet solution.)

(3) The spectrum of the operator $H$ coincides with the projection of its (real) dispersion relation onto the spectral $\lambda$-axis.

(4) If the branches of the multiple valued function $k \in \mathbb{R} \mapsto \lambda \in \mathbb{R}$ are labeled in increasing order $\left(\lambda_{1}(k) \leq \lambda_{2}(k) \leq \cdots\right)$, then the range of the $j$ th branch is the $j$ th spectral band of $H$.

(5) In terms of the discriminant, the dispersion relation (both in the real and complex incarnations) is described as the set of all (correspondingly real or complex) solutions $(k, \lambda)$ of the equation

$$
\Delta(\lambda)-2 \cos k=0 .
$$

In other words, it is the graph of the multiple valued function

$$
\lambda=\Delta^{-1}(2 \cos k) .
$$

Formula (1.21) is less useful than (1.20), due to its multiple valued (and branching) nature. On the other hand, the function $F(\lambda, k):=\Delta(\lambda)-2 \cos k$ is an entire function in $\mathbb{C}^{2}$ of a finite exponential order (equal to 1 in Hill's case). In particular, one can make the following observation, which gains prominence in the PDE situation:

Proposition 1.15. The dispersion relation of the Hill operator is a principal (i.e., of codimension 1) analytic subset in $\mathbb{C}^{2}$.

Let us look at the case of the free operator. Then the dispersion relation boils down to $\cos \sqrt{\lambda}=\cos k$, or to the union of infinitely many parabolic branches

$$
\lambda=(k+2 n \pi)^{2}, n \in \mathbb{Z} ;
$$

see Figure 4. Due to the periodicity with respect to $k$, it is customary to draw

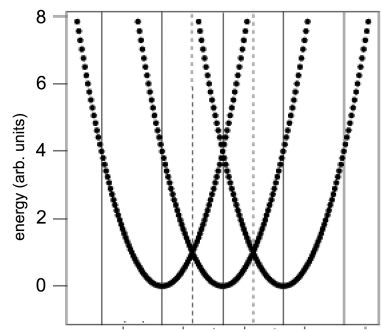

Free: secret revealed

FIGURE 4. The dispersion relation (over the whole quasimomentum line) of the free operator. 


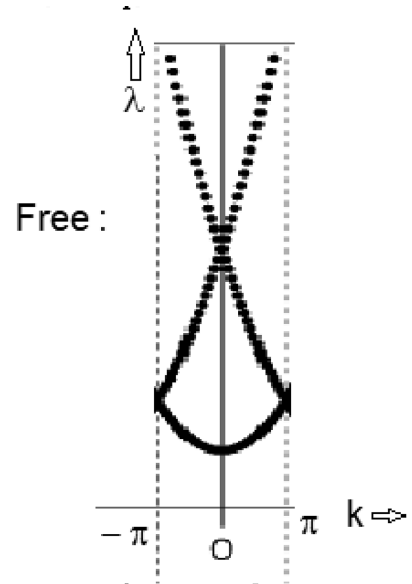

Brillouin Zone

FiguRE 5. The dispersion relation of the free operator.

the dispersion relation only over the interval $[-\pi, \pi]$ (below, this interval will be called a Brillouin zone). In fact, due to the evenness, one can, without loosing any information, restrict to $[0, \pi]$ (reduced Brillouin zone) only (see Figure 5).

One can see that the seeming complexity of the dispersion relation, even in the free case, is an illusion, since one observes a single period view of a periodic sequence of parabolas.

Let us see how the picture gets perturbed when a small periodic potential $V(x)$ is turned on (Figure 66). You see that under this perturbation some gaps are opening. However, it seems that over $[0, \pi]$ each branch is still monotonic. Let us see why this is indeed true.

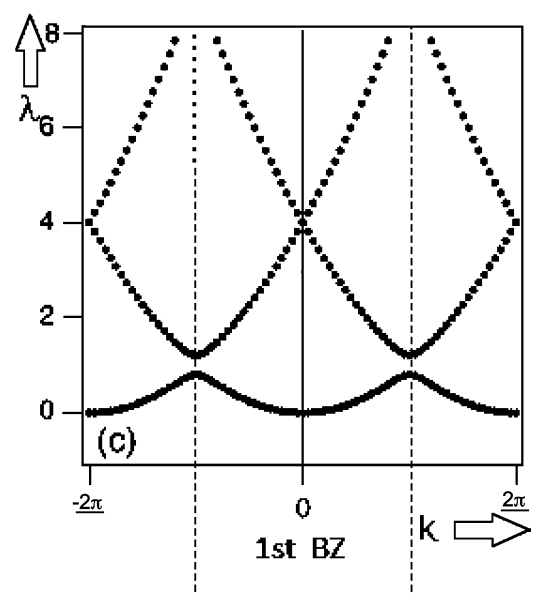

Near free

Figure 6. The dispersion relation in presence of a potential. 


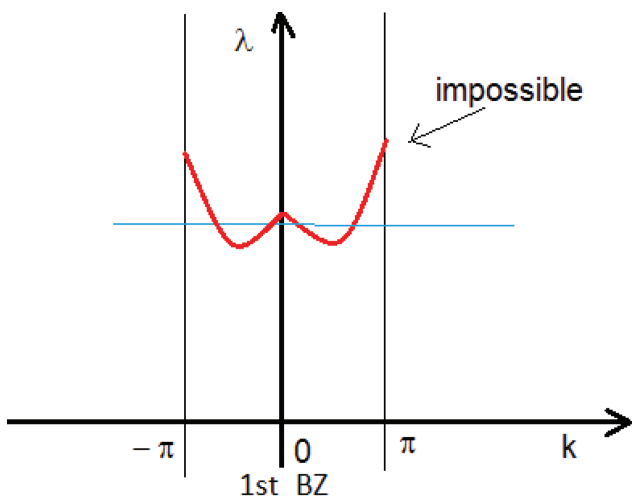

Figure 7. Nonmonotonicity is impossible.

\section{Lemma 1.16.}

(1) Each branch $\lambda_{j}(k)$ of the dispersion relation is monotonic over $[0, \pi]$.

(2) In particular, the spectral band edges occur only at $k=0$ and $k=\pi$, i.e., for periodic or anti-periodic problems for the Hill operator.

Proof. Indeed, if this were not true, taking into account the evenness with respect to $k$, one would have a situation such as that sown in Figure 7 If we pick the value of $\lambda$ at the level shown, we will find four values of $k$ where this level is reached. This means that the equation $H u=\lambda u$ has four independent solutions. The ODE being of the second order, this is impossible.

The same argument shows the impossibility of band overlaps, as in Figure 8 . Thus, one concludes:

Lemma 1.17. The spectral bands of the Hill operator do not overlap (although they might touch).

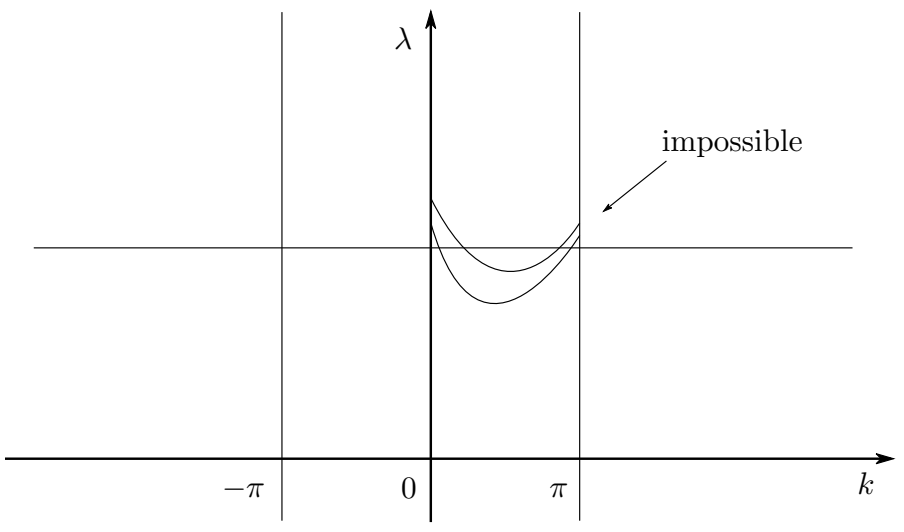

Figure 8. No band overlap. 
Remark 1.18. Notice that the conclusions about monotonicity, band edges occurring at $k=0$ and $k=\pi$ only, and the absence of band overlap were drawn from the fact that the second-order ODE cannot have more than two independent solutions. One can wonder whether this is just an artifact of our proof or something deeper. For instance, this counting cannot be used for PDEs. And indeed, we will see that for PDEs all these claims are in general incorrect.

We formulate now without proofs some additional spectral properties:

\section{Theorem 1.19.}

(1) The pure point and singular continuous spectra of the Hill operator are empty. Thus, the spectrum is absolutely continuou 6 [117,244, 332].

(2) The dispersion relation, as an analytic set, is irreducible (modulo the $2 \pi$ shifts of the quasimomentum). In other words, any open part of the dispersion relation uniquely determines the whole of it. To put it still differently, the smooth part of $B_{H}$ is connected [231; see also [16]. (See [316] for additional irreducibility results for periodic ODEs.)

(3) The Bloch variety (which is analytic, as we know), is generically not algebraic (e.g., [282, 283]).

\section{LATTICES IN $\mathbb{R}^{n}$}

Switching to the multiperiodic case, we first need to go through some basics concerning lattices. One can find a nice more detailed introduction in 358.

Definition 2.1. A (Bravais) lattice $\Gamma$ in $\mathbb{R}^{n}$ is the set of all integer linear combinations of $n$ linearly independent vectors $a_{1}, \ldots, a_{n}$ (see Figure 9):

$$
\Gamma=\left\{\gamma \in \mathbb{R}^{n} \mid \gamma=\sum_{j=1}^{n} \gamma_{j} a_{j}, \gamma_{j} \in \mathbb{Z}\right\} .
$$

We will identify $\Gamma$ with the corresponding group of shifts of $\mathbb{R}^{n}$.

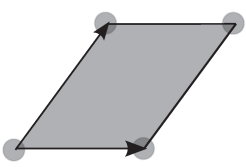

Figure 9. A lattice and its fundamental domain (shaded).

Definition 2.2. The dual (reciprocal) lattice to $\Gamma, \Gamma^{*} \subset\left(\mathbb{R}^{n}\right)^{*}$ (see Figure 10) is defined as follows:

$$
\Gamma^{*}=\left\{k \in\left(\mathbb{R}^{n}\right)^{*} \mid\langle k, \gamma\rangle \in 2 \pi \mathbb{Z} \text { for any } \gamma \in \Gamma\right\} .
$$

The original lattice $\Gamma$ is sometimes called the real lattice.

\footnotetext{
${ }^{6}$ We will discus this at length for the PDE case.
} 


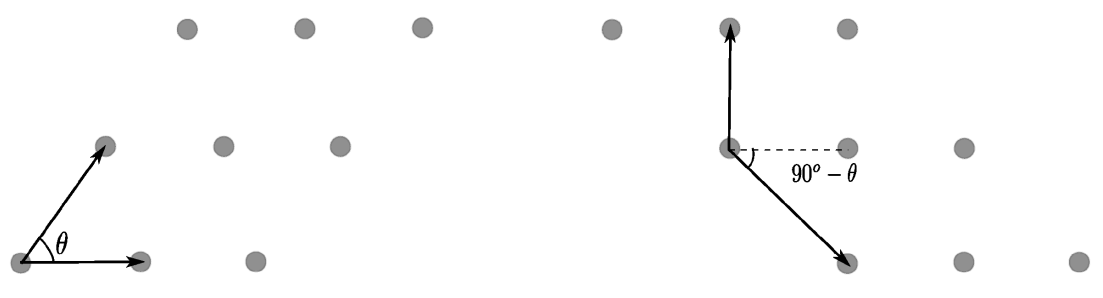

FiguRE 10. A real and its reciprocal lattices.

In particular, when $\Gamma=\mathbb{Z}^{n}$ and the duality is coming from the standard Euclidean scalar product, then $\Gamma^{*}=2 \pi \mathbb{Z}^{n}$.

Definition 2.3. We fix a connected fundamental domain $W$ (Wigner-Seitz cell) of the (real) lattice $\Gamma$ in $\mathbb{R}^{n}$, i.e., a closed domain such that its $\Gamma$-shifts may intersect only along their boundaries and cover the whole space (see Figure 9) 7

We will also fix a fundamental domain $\mathcal{B}$ of (the reciprocal lattice) $\Gamma^{*}$ in $\left(\mathbb{R}^{n}\right)^{*}$, which we will call the Brillouin zone. One of the options (standard in physics) is to choose the Voronoi cell, i.e., the set of all points such that the origin is the closest point to them in $\Gamma^{*}$ (see Figure 11). In this work, we will be choosing a parallelepiped as the fundamental domain (see (2.3) ) and also skip the consideration of important so-called second, third, etc., Brillouin zones (see, e.g., 71, 358 for definitions and interesting discussions).

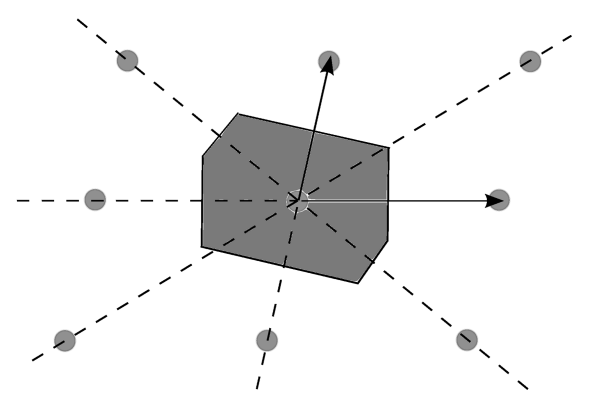

Figure 11. A Brillouin zone of a lattice.

The particular choice of a lattice does not matter in many, and does matter in some results, but for the sake of simplicity, we will assume, unless noted otherwise, that

$$
\Gamma=\mathbb{Z}^{n}, \Gamma^{*}=2 \pi \mathbb{Z}^{n}, W=[0,1]^{n}, \mathcal{B}=[-\pi, \pi]^{n} .
$$

We also introduce two tori that correspond to the two lattices

$$
\mathbb{T}:=\mathbb{R}^{n} / \Gamma \text { and } \mathbb{T}^{*}:=\left(\mathbb{R}^{n}\right)^{*} / \Gamma^{*},
$$

equipped with normalized Haar measures on both; e.g., under our standard choice (2.3), the measures are correspondingly $d x$ and $(2 \pi)^{-n} d k$.

\footnotetext{
${ }^{7} \mathrm{~A}$ fundamental domain is clearly not defined uniquely.
} 
Note that under our assumption that $\Gamma=\mathbb{Z}^{n}$, the torus $\mathbb{T}^{*}$ can be considered as the unit torus in $\mathbb{C}^{n}$ :

$$
\mathbb{T}^{*}=\left\{\left(z_{1}, \ldots, z_{n}\right) \in \mathbb{C}^{n}|| z_{1}|=\cdots=| z_{n} \mid=1\right\} .
$$

As usual, $\Gamma$-periodic (resp. $\Gamma^{*}$-periodic) functions on $\mathbb{R}^{n}\left(\right.$ on $\left.\left(\mathbb{R}^{n}\right)^{*}\right)$ can be identified with functions on the torus $\mathbb{T}\left(\mathbb{T}^{*}\right)$.

Fourier series (FS) identify $L^{2}(\mathbb{T})$ with the $l^{2}$-space on $\Gamma^{*}$ :

$$
f(x) \mapsto\left\{f_{k}:=\int_{\mathbb{T}} f(x) e^{-i k \cdot x} d x\right\}_{k \in \Gamma^{*}} .
$$

Analogously with $L^{2}\left(\mathbb{T}^{*}\right)$ and $l^{2}$ on $\Gamma$.

One can also consider vector-valued Fourier series. Namely, let $\mathcal{H}$ be a Hilbert space, and let $L^{2}(\mathbb{T}, \mathcal{H}), l^{2}\left(\Gamma^{*}, \mathcal{H}\right)$ be the spaces of $\mathcal{H}$-valued $L^{2}$-functions on $\mathbb{T}$ and $\Gamma^{*}$ correspondingly and the Fourier series expansion is defined as in (2.6). The following theorem restates standard results concerning Fourier series:

\section{Theorem 2.4.}

- The FS expansion is an isometry of $L^{2}(\mathbb{T}, \mathcal{H})$ onto $l^{2}\left(\Gamma^{*}, \mathcal{H}\right)$.

- A function $\in L^{2}(\mathbb{T}, \mathcal{H})$ is infinitely differentiable if and only if the norms $\left\|f_{k}\right\|$ of its Fourier coefficients decay faster than any power of $k$.

- A function $\in L^{2}(\mathbb{T}, \mathcal{H})$ allows analytic continuation into a complex neighborhood of $\mathbb{T}$ (see (2.5)) if and only if the norms $\left\|f_{k}\right\|$ of its Fourier coefficients decay exponentially.

\section{Periodic operators}

From now on, we will be interested in studying linear elliptic partial different operators with periodic coefficients. Our main "test" example is the Schrödinger operator in $L^{2}\left(\mathbb{R}^{n}\right)$

$$
H=-\Delta+V(x)
$$

with a "sufficiently nice" real electric potential $V$, periodic with respect to the group $\Gamma=\mathbb{Z}^{n}$ (and thus the dual lattice is $\Gamma^{*}=2 \pi \mathbb{Z}^{n}$ ). The domain of $H$ is the Sobolev space $H^{2}\left(\mathbb{R}^{n}\right)$. In most cases, the reader will not be misled by thinking just of this operator.

Many other periodic operators of mathematical physics arise in applications and need to be studied. The techniques described in this survey work (sometimes with some caveats) for them as well. We will from time to time address some of those, but so far we just briefly describe some examples worthy of studying (and being studied):

- Although we assumed above a nice periodic potential $V(x)$ (continuous or even smooth), this condition is unnecessarily strong (e.g., $L_{2, \text { loc }}$ usually suffices). However, the main issues already arise in the smooth case. So, unless specified otherwise, coefficients in all operators will be assumed sufficiently smooth (e.g., $C^{\infty}$ ), albeit in practical applications they often are nonsmooth, even discontinuous.

- The Schrödinger operator (3.1) is self-adjoint, however many of the techniques and results do not require self-adjointness. For instance, when we discuss in Section 6.3 absolute continuity of the spectrum, we will 
restate it as the absence of the pure point spectrum. In this form, it holds, without any change in the proof, in the non-self-adjoint setting as well.

- More general Schrödinger operators are also of interest and present more difficulties in studying them. These are, first of all, the magnetic Schrödinger operator

$$
\left(\frac{1}{i} \nabla-A(x)\right)^{2}+V(x)
$$

with periodic magnetic and electric potentials $A(x)$ and $V(x)$ (one assumes the gauge $\nabla \cdot A=0)$.

One encounters even more difficulties in the presence of a periodic metric

$$
-\nabla \cdot g(x) \nabla+i A(x) \cdot \nabla+V(x),
$$

where $g(x)$ is a periodic positive definite matrix function.

- Periodic elliptic operators of higher than second order are also of some interest. Here, one should beware that such operators, unlike the secondorder ones, might not obey even the weakest uniqueness of continuation laws, which influences the validity of some of the results (e.g., absence of the pure point spectrum).

- Periodic elliptic systems (including overdetermined ones) can also be considered, first of all the Maxwell operator (in its second-order incarnation)

$$
\nabla \times \varepsilon^{-1}(x) \nabla \times
$$

where $\varepsilon(x)$ is a periodic positively definite scalar function (or tensor) and $\nabla \times$ is the curl operator. Here one encounters an additional difficulty, since this operator is not elliptic by itself, but only as a member of an elliptic complex of operators.

- The natural mapping $\mathbb{R}^{n} \mapsto \mathbb{T}$ is a normal abelian covering of the torus $\mathbb{T}$. The techniques that we will consider, as well as many results, apply to periodic operators on coverings

$$
M \mapsto N,
$$

subject to the following three conditions: (1) The base $N$ is compact; (2) The deck group $\Gamma$ of the covering is finitely generated and virtually abelian 8

(3) The operator on $M$ is "elliptic" in the sense that being pushed down to $N$, it is a Fredholm operator in appropriate spaces.

In particular, periodic operators on abelian coverings of compact Riemannian manifolds, analytic manifolds, and even graphs succumb gladly to the theory.

- Elliptic periodic boundary value problems arise in various applications (e.g., waveguides and photonic crystals). Here periodicity is imposed on the shape of the domain, coefficients of the operator, and boundary conditions. Some issues easily resolved for operators in the whole space become much harder in this new setting (see, e.g., Section 9.4).

- Ellipticity conditions can be weakened to hypoellipticity, e.g., parabolicity, although the known results are much weaker here (see Section 9.1 and [244, Ch. 5, and references therein]).

\footnotetext{
${ }^{8}$ That is, it contains a finite index abelian subgroup.
} 
- Nonhypoelliptic periodic equations, e.g., important time periodic hyperbolic or nonstationary Schrödinger equations require different techniques, due to a lack of the Fredholm property (see, e.g., 396 398] and Section 9.1).

- Some, but not all, studies carry over to periodic elliptic pseudo-differential operators ( $\Psi$ DOs) (see, e.g., [244, Section 3.4.C], [323, 367, and references therein).

\section{Floquet transform and Direct integral DECOMposition}

Our main tools in 1D were the spectral analysis of the monodromy operator and the Lyapunov reduction theorem. Both of them rely upon the propagator along the solutions of the Cauchy problem. This raises some hopes that an analog of the 1D Floquet theory might work for time-periodic PDEs of evolution type (parabolic, hyperbolic, nonstationary Schrödinger), where such propagators are nicely defined. And indeed, sometimes this does happen, although, surprisingly (and shamefully) some basic issues still remain unresolved (see some details in Section 9.1). However, for nonevolution (e.g., elliptic) periodic PDEs using such propagators is all but impossible $*$. One thus has to resort to a different technique, which we outline below.

4.1. Floquet transform. As we have recalled above, Fourier series transform functions of $\gamma \in \Gamma$ (even with values in a Hilbert space) into functions of $z \in \mathbb{T}^{*}$

$$
\left\{f_{\gamma}\right\}_{\gamma \in \Gamma} \mapsto \widehat{f}(z):=\sum_{\gamma \in \Gamma} f_{\gamma} z^{\gamma}=\sum_{\gamma \in \Gamma} f_{\gamma} e^{i k \cdot \gamma}
$$

with standard $L^{2}$ isometry and Paley-Wiener type theorems being preserved. Here $k \in \mathbb{R}^{n}$ and $z$ belongs to the unit torus $\mathbb{T}^{*}$.

Now let $f(x)$ be a function in $L_{\text {loc }}^{2}\left(\mathbb{R}^{n}\right)$. We cover $\mathbb{R}^{n}$ by the shifted copies of the fundamental domain $W$ :

$$
\mathbb{R}^{n}=\bigcup_{\gamma \in \Gamma}(W-\gamma)
$$

cut $f$ into the corresponding pieces $\left.f\right|_{W-\gamma}$ and then shift them all back to the original $W$

$$
f_{\gamma}(x):=\left.f\right|_{W-\gamma}(x-\gamma)
$$

Here if we restrict $x$ to being in the Wigner-Seitz cell $W$, we get a function on $\Gamma$ with values in the Hilbert space $L^{2}(W)$

$$
\gamma \in \Gamma \mapsto f_{\gamma} \in L^{2}(W) .
$$

Now summing the Fourier series, we arrive to our main tool: 
Definition 4.1. The Floquet transform $9 \mathcal{U}_{\Gamma}$ acts as follows:

$$
f(x) \mapsto \mathcal{U}_{\Gamma} f(x, k):=\sum_{\gamma \in \Gamma} f_{\gamma}(x) e^{i k \cdot \gamma}=\sum_{\gamma \in \Gamma} f_{\gamma}(x) z^{\gamma} ;
$$

i.e., this is just the sum of the Fourier series, whose coefficients are the pieces of $f$ over the shifted copies of the fundamental domain.

Here, as before, $z=\left(z_{1}, \ldots, z_{n}\right):=\left(e^{i k_{1}}, \ldots, e^{i k_{n}}\right)$ is the Floquet multiplier corresponding to the crystal momentum $k=\left(k_{1}, \ldots, k_{n}\right)$. We will also abuse notation, writing

$$
\mathcal{U}_{\Gamma} f(x, z)=\sum_{\gamma \in \Gamma} f_{\gamma}(x) z^{\gamma} .
$$

It will be convenient to extend $\mathcal{U}_{\Gamma} f(x, k)$ from $x \in W$ to the whole $\mathbb{R}^{n}$ just by removing the restriction to $W-\gamma$ in (4.2), which results in

$$
f(x) \mapsto \mathcal{U}_{\Gamma} f(x, k):=\sum_{\gamma \in \Gamma} f(x-\gamma) e^{i k \cdot \gamma}=\sum_{\gamma \in \Gamma} f(x-\gamma) z^{\gamma} .
$$

If this is done, then one observes the following useful properties:

$$
\begin{gathered}
\mathcal{U}_{\Gamma} f(x+\gamma, k)=\mathcal{U}_{\Gamma} f(x, k) e^{i k \cdot \gamma}, \gamma \in \Gamma, \\
\mathcal{U}_{\Gamma} f(x+\gamma, z)=\mathcal{U}_{\Gamma} f(x, z) z^{\gamma}, \gamma \in \Gamma, \\
\mathcal{U}_{\Gamma} f\left(x, k+k^{\prime}\right)=\mathcal{U}_{\Gamma} f(x, k), k^{\prime} \in \Gamma^{*}
\end{gathered}
$$

The first two equalities, (4.6) and (4.7), say that, being considered on the whole space $\mathbb{R}^{n}, \mathcal{U}_{\Gamma} f(x, k)$ is $\Gamma$-automorphic with respect to $x$ with the character $e^{i k \cdot \gamma}$ (this is also called cyclic property, quasiperiodicity, or Floquet property). The last one, (4.8) claims $\Gamma^{*}$-periodicity with respect to $k$.

It is easy to get an inversion formula for the Floquet transform. Indeed, it is enough to find the Fourier coefficients of $\mathcal{U}_{\Gamma} f(x, k)$ with respect to $k$ and place them where they belong, i.e., on the shifted copies of the fundamental domain. This leads to the following inversion formula:

$$
f(x)=\int_{\mathbb{T}^{*}} \mathcal{U}_{\Gamma} f(x, k) e^{-i k \cdot \gamma} d k, x \in W-\gamma, \text { for any } \gamma \in \Gamma .
$$

On the other hand, if we consider the Fourier coefficients $f_{\gamma}$ as the shifted copies of $f$ in the whole space $\mathbb{R}^{n}$, then just the zero's coefficient recovers the whole function

$$
f(x)=\int_{\mathbb{T}^{*}} \mathcal{U}_{\Gamma} f(x, k) d k, x \in \mathbb{R}^{n} .
$$

\footnotetext{
${ }^{9}$ Neither the name "Floquet transform" is commonly accepted nor did Floquet introduce this transform. Various other names have been suggested: Bloch transform, Gelfand transform (due to [166]), Zak transform, and probably some others. Here we see an instance of the so-called Arnold's principle: If a notion bears a personal name, then this name is not the name of the discoverer [9]. (See also Berry's principle: Arnold's principle is applicable to itself and Stigler's law of eponymy 369.) However, it is handy to use a name, rather than point a finger to a formula. Thus, the author chose Floquet, since this transform is very natural for Floquet theory.
} 
4.2. Plancherel and Paley-Wiener type theorems. Now, application of Theorem 2.4 to $\mathcal{H}=L^{2}(W)$ leads to the following range theorems for the Floquet transform.

\section{Theorem 4.2.}

- $\mathcal{U}_{\Gamma}$ is isometry from $L^{2}\left(\mathbb{R}^{n}\right)$ onto $L^{2}\left(\mathbb{T}^{*}, L^{2}(W)\right)$.

- $\mathcal{U}_{\Gamma} f(\cdot, k)$ is infinitely differentiable on $\mathbb{T}^{*}$ as a function with values in $L^{2}(W)$ iff the norms $\|f\|_{L^{2}(W+\gamma)}$ decay when $|\gamma| \mapsto \infty$ faster than any power of $|\gamma|$.

- $\mathcal{U}_{\Gamma} f(\cdot, k)$ is analytic in a complex neighborhood of $\mathbb{T}^{*}$ as a function with values in $L^{2}(W)$ iff the norms $\|f\|_{L^{2}(W+\gamma)}$ decay exponentially fast when $|\gamma| \mapsto \infty$.

Dealing with differential operators, we will also be interested in the behavior of Sobolev spaces $H^{s}\left(\mathbb{R}^{n}\right)$ under the Floquet transform. This seems to be a "piece of cake", just replacing everywhere $L^{2}$ with $H^{s}$. Well, not so fast:

Proposition 4.3. $\mathcal{U}_{\Gamma}$ is an isometry from $H^{s}\left(\mathbb{R}^{n}\right)$ onto a proper subspace of $L^{2}\left(\mathbb{T}^{*}, H^{s}(W)\right)$.

Oops! What is wrong with Sobolev spaces? The answer is not hard to figure out. Indeed, if one takes an arbitrary function $F(x, k)$ in $L^{2}\left(\mathbb{T}^{*}, H^{s}(W)\right)$, its alleged preimage under the Floquet transform can be, as we have figured out before, recovered by taking Fourier coefficients of $F$ and distributing them to the appropriate shifts of the fundamental domain $W$. The resulting function is, by construction, in $H^{s}$ inside of each of the shifted copies of $W$. Moreover, the sum of squares of their $H^{s}$-norms is finite. The problem is that there is nothing enforcing the smoothness across the boundary between two adjacent cells.

Fortunately, it is not hard to fix this and to describe the range of the transform explicitly. Clearly, some boundary conditions are needed to glue the pieces together. And indeed these are easy to establish, for instance, when $s=2$ (or any $s \in \mathbb{Z}^{+}$). It is, however, easier to avoid this and to adopt a much more telling (and applicable for any $s \geq 0$ ) view. The clue on how to do this comes from the automorphicity property 44.6, 4.7). We introduce the following

Definition 4.4. For any $k \in \mathbb{C}^{n}$, the space $H_{k}^{s}(W)$ is the space of restrictions to $W$ of the $H_{\mathrm{loc}}^{s}$-function on $\mathbb{R}^{n}$ that is automorphic with the given $k$ :

$$
H_{k}^{s}(W):=\left\{\left.f\right|_{W} \mid f \in H_{\mathrm{loc}}^{s}\left(\mathbb{R}^{n}\right), f(x+\gamma)=e^{i k \cdot \gamma} f(x) \text { a.e., } \forall \gamma \in \Gamma\right\} .
$$

Another useful object is a $k$-dependent smooth line bundle over $\mathbb{T}$. Let us fix $k \in \mathbb{C}^{n}$ and consider first the trivial line bundle $E:=\mathbb{R}^{n} \times \mathbb{C}$ over $\mathbb{R}^{n}$. We define a ( $k$-dependent) $\Gamma$-action on $E$ as follows:

$$
\text { for any } \gamma \in \Gamma \text { and }(x, c) \in E, \tau_{k}(\gamma)(x, c):=\left(x+\gamma, e^{i k \cdot x} c\right) \text {. }
$$

Definition 4.5. We denote by $E_{k}$ the line bundle over the torus $\mathbb{T}$ obtained by factoring out the action $\tau_{k}$ of $\Gamma$ on the bundle $E$.

Now the following claims are immediate to obtain:

Proposition 4.6. Let $s \geq 0$. Then, for any $k$,

(1) $H_{k}^{s}$ is a closed subspace in $H^{s}(W)$.

(2) $H_{k}^{0}=L_{k}^{2}=L^{2}(W)$. 
(3) $H_{k+k^{\prime}}^{s}=H_{k}^{s}$ for any $k^{\prime} \in \Gamma^{*}$.

(4) A function $u$ belongs to $H_{k}^{s}$ iff

$$
u(x)=e^{i k \cdot x} v(x) \text { with } v(x) \text { being } \Gamma \text {-periodic, i.e., } v \in H^{s}(\mathbb{T}) .
$$

(5) Moreover, $\mathcal{H}^{s}:=\bigcup_{k} H^{s}(k)$ is an analytic Banach vector subbundle of the trivial bundle $\mathbb{C}^{n} \times H^{s}(W)$ over $\mathbb{C}^{n}$.

(6) There is a natural correspondence between $\Gamma$-automorphic functions on $\mathbb{R}^{n}$ with a quasimomentum $k$ and sections of the linear bundle $E_{k}$ over $\mathbb{T}$.

(7) Under this correspondence, elements of $H_{k}^{s}$ correspond to $H^{s}$-sections of the bundle $E_{k}$.

(8) The operator $H$ preserves the $\Gamma$-automorphicity. In particular, $H$ defines an elliptic operator $H(k)$ on sections of the bundle $E_{k}$.

Remark 4.7.

- In the particular case when $H$ is the Laplacian $-\Delta$, the operators $-\Delta(k)$ are called twisted Laplacians. We call $H(k)$ twisted Schrödinger operators, or (more commonly) Bloch Hamiltonians.

- The advantage of dealing with the family of operators $H(k)$ rather than the single operator $H$ is that each $H(k)$, being an elliptic operator in sections of a bundle over a compact manifold $\mathbb{T}$, has purely discrete spectrum, unlike the operator $H$, whose spectrum is continuous.

- For the operator $H=-\Delta+V(x)$, the corresponding $H(k)$ acts by the same differential expression on $W$ with the domain $H_{k}^{2}$, i.e., with the cyclic boundary conditions. In this view, the domain of the operator analytically rotates with $k$, while the differential expression of $H$ stays the same. One of its advantages is that the domain $H_{k}^{s}$ of the operator is $\Gamma^{*}$-periodic with respect to $k$, so it can be considered as depending on the Floquet multiplier $z=\left(e^{i k_{1}}, \ldots, e^{i k_{n}}\right)$ only.

- Alternatively, according to (4.13), the operator $H(k)$ can be considered as acting, for all $k$, on periodic functions on $\mathbb{T}$. This is achieved by commuting with an exponential function

$$
e^{-i k \cdot x} \circ(-\Delta+V(x)) \circ e^{i k \cdot x}
$$

This leads to the following differential expression for $H(k)$

$$
-\Delta-2 i k \cdot \nabla+k^{2}+V(x)
$$

acting on the torus $\mathbb{T}$, i.e., on $\Gamma$-periodic functions. The advantage of this representation is the explicit polynomial dependence of the differential expression on the quasimomentum and the domain being fixed. On the other hand, explicit periodicity in $k$ is lost.

There are advantages and disadvantages of both representations. It is thus recommended to choose them judiciously, depending on the problem.

- The latter representation can be generalized to any periodic differential operator $L(x, D)$, where $D:=\frac{1}{i} \frac{\partial}{\partial x}$ :

$$
L(k)=L(x, D+k) .
$$

We can now formulate the exact range theorem for the Floquet transform in Sobolev spaces: 
Theorem 4.8.

- $\mathcal{U}_{\Gamma}$ is an isometry from $H^{s}\left(\mathbb{R}^{n}\right)$ onto the space $L^{2}\left(\mathbb{T}^{*}, \mathcal{H}^{s}\right)$ of the $L^{2}$-sections of the bundle $\mathcal{H}^{s}$.

- The section $\mathcal{U}_{\Gamma} f(\cdot, k)$ is infinitely differentiable on $\mathbb{T}^{*}$ iff the norms $\|f\|_{H^{s}(W+\gamma)}$ decay when $\|\gamma\| \mapsto \infty$ faster than any power of $\|\gamma\|$.

- The section $\mathcal{U}_{\Gamma} f(\cdot, k)$ is analytic in a complex neighborhood of $\mathbb{T}^{*}$ iff the norms $\|f\|_{H^{s}(W+\gamma)}$ decay exponentially fast when $\|\gamma\| \mapsto \infty$.

4.3. Direct integral decomposition. We can summarize the results of previous sections as the following direct integral decompositions of functional spaces and of the operator $H$ :

$$
L^{2}\left(\mathbb{R}^{n}\right)=\int_{\mathbb{T}^{*}}^{\oplus} L^{2}(W), \quad H^{s}\left(\mathbb{R}^{n}\right)=\int_{\mathbb{T}^{*}}^{\oplus} H_{k}^{s}, \quad H=\int_{\mathbb{T}^{*}}^{\oplus} H(k) .
$$

We will not get into any deeper discussions of the direct integral technique (see, e.g., 19, 102, 154, 303, 332, 338).

\section{Dispersion RELATion AND ALL THAT}

As there is in general no analog of the $1 \mathrm{D}$ discriminant in higher dimensions, the dispersion relation takes the lead. Its definition and many properties are analogous to the ones in $1 \mathrm{D}$, although some important distinctions do arise.

\subsection{Dispersion relation $=$ Bloch variety .}

Definition 5.1. The (real) dispersion relation (or the (real) Bloch variety $\left.B_{H}\right)$ of the periodic Schrödinger operator $10 H$ is the subset of $\mathbb{R}_{k}^{n} \times \mathbb{R}_{\lambda}$ defined as follows:

$B_{H}:=\left\{(k, \lambda) \in \mathbb{R}^{n+1} \mid H u=\lambda u\right.$ has a nontrivial Bloch-Floquet solution $u(x)=e^{i k \cdot x} p(x)$ with the quasimomentum $\left.k\right\}$.

The complex dispersion relation (or complex Bloch variety) is defined analogously, only allowing both $k$ and $\lambda$ to be complex, i.e., $B_{H, \mathbb{C}} \subset \mathbb{C}^{n+1}$. As we will see later on, the ability of considering the complex Bloch variety turns out to be very important.

5.2. Dispersion relation and the spectrum. Let us assume that $k \in \mathbb{R}^{n}$. The operator $H(k)$ being a self-adjoint elliptic operator in sections of a linear bundle on the torus, implies:

Lemma 5.2. For any $k \in \mathbb{R}^{n}$, the operator $H(k)$ is bounded below and has a discrete (real) spectrum

$$
\sigma(H(k))=\left\{\lambda_{j}(k) \mid \lambda_{1}(k) \leq \lambda_{2}(k) \leq \cdots \leq \lambda_{d}(k) \leq \cdots \mapsto \infty\right\} .
$$

Definition 5.3. The function $\lambda_{j}(k)$ is called the $j$ th band function.

Remark 5.4. Notice that for complex quasimomenta $k$, the spectrum of $H=$ $-\Delta(k)+V$ is also discrete [4, pp. 180-190], although labeling the band functions becomes an issue.

\footnotetext{
${ }^{10}$ The definition stays the same for other periodic operators.
} 
The following statements hold, due to the direct integral expansion (4.17) of the operator, perturbation theory [217], and standard properties of analytic Fredholm operator functions (e.g., [244, 394, 404]):

\section{Theorem 5.5.}

(1) The band functions are continuous and piecewise analytic.

(2) The graph of the multiple valued mapping

$$
k \in \mathbb{R}^{n} \mapsto \sigma(H(k))
$$

coincides with the dispersion relation (Bloch variety) $B_{H}$ of $H$.

(3) The latter claim also holds in the complex case, i.e., the graph of the multiple valued mapping

$$
k \in \mathbb{C}^{n} \mapsto \sigma(H(k))
$$

coincides with the complex dispersion relation (complex Bloch variety) of $H$.

(4) The dispersion relation is $\Gamma^{*}$-periodic with respect to $k$ and thus it is sufficient to consider it only over the Brillouin zone $\mathcal{B}$.

(5) The dispersion relation is symmetric (even) with respect to the mapping $k \mapsto-k$. (This fails if the potential is not real.)

(6) The spectrum $\sigma(H)$ is the range of the (real) dispersion relation, i.e.,

$$
\begin{aligned}
\sigma(H) & =\bigcup_{k \in \mathcal{B}} \sigma(H(k)) \\
& =\left\{\lambda \in \mathbb{R} \mid \exists k \in \mathbb{R}^{n}, \text { such that } \lambda \in \sigma(H(k))\right\} \\
& =\left\{\lambda \in \mathbb{R} \mid \exists k \in \mathbb{R}^{n}, j \in \mathbb{Z}^{+} \text {, such that } \lambda=\lambda_{j}(k)\right\} .
\end{aligned}
$$

The last statement of the theorem is a very general claim about spectra of direct integrals of operators (see, e.g., 244,332).

In Figure 12 one finds an example of the dispersion relation for a nonzero potential.

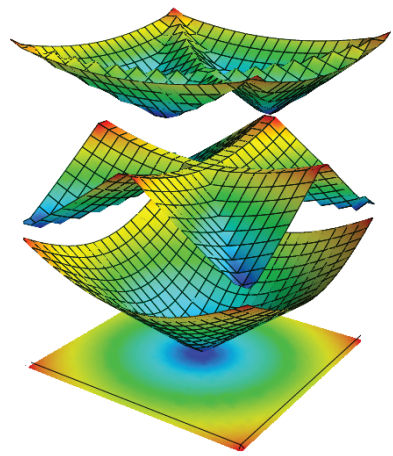

FiguRE 12. The dispersion relation of a nonfree operator, drawn over a Brillouin zone. 
The following lemma is often very useful:

Lemma 5.6. Let $S$ be a dense subset of the Brillouin zone $\mathcal{B}$. Then

$$
\sigma(H)=\overline{\bigcup_{k \in S} \sigma(H(k))} .
$$

In other words, if for some reason one wants to avoid some exceptional values of $k$ (e.g., $k=0$ causes troubles for the Maxwell operator), one can avoid them by choosing an appropriate dense subset of quasimomenta.

We can now define the spectral bands of the operator $H$ :

Definition 5.7. The segment

$$
I_{j}:=\operatorname{range}\left(\lambda_{j}\right)=\left\{\lambda \in \mathbb{R} \mid \exists k \in \mathbb{R}^{n} \text { such that } \lambda=\lambda_{j}(k)\right\}
$$

is the $j$ th band of the spectrum $\sigma(H)$.

Due to $\Gamma^{*}$-periodicity with respect to $k$, one can also write

$$
I_{j}=\left\{\lambda \in \mathbb{R} \mid \exists k \in \mathcal{B} \text { such that } \lambda=\lambda_{j}(k)\right\} .
$$

\section{Corollary 5.8.}

(1) Each band $I_{j}$ is a finite closed interval, both of whose endpoints tend to infinity when $j \rightarrow \infty$.

(2) The bands cover the whole spectrum:

$$
\sigma(H)=\bigcup_{j \in \mathbb{Z}^{+}} I_{j}
$$

(3) The bands cant11 overlap.

As it was in $1 \mathrm{D}$, it is easy to check that for the free operator $H=-\Delta, B_{H}$ is the union of the paraboloid $\lambda=k^{2}$ and its $\Gamma^{*}$-shifts. One can deduce from this (which requires highly nontrivial work involving number theory (e.g., 317, 323, 358,)) the following two theorems:

Theorem 5.9. Consecutive spectral bands of the free operator overlap, and thus the spectrum of the free operator has no gaps.

This is in a stark contrast with $1 \mathrm{D}$, where the bands do not overlap (touching in the worst case).

To quantify the overlap of the bands $I_{j}$, Skriganov (see, e.g., 358]) introduced the following continuous overlap function on $\mathbb{R}$ :

\section{Definition 5.10.}

$$
\zeta(\lambda):=\left\{\begin{array}{l}
\max _{j} \max \left\{t \geq 0 \mid[\lambda-t, \lambda+t] \subset I_{j}\right\}, \lambda \in \sigma(H) \\
0, \lambda \notin \sigma(H)
\end{array} .\right.
$$

\section{Theorem 5.11.}

(1) In dimension $n=2$, one has

$$
\lim _{\lambda \rightarrow \infty} \zeta(\lambda)=\infty
$$

\footnotetext{
${ }^{11}$ And, as we will see, usually do.
} 
(2) When $n=3$,

$$
\lim \sup _{\lambda \rightarrow \infty} \zeta(\lambda) \geq c>0 .
$$

(3) In dimensions $n \geq 4$, the lower bounds for the overlap functions are given by negative powers of $\lambda$ (see 323 for details and further references).

5.3. Periodic operators as perturbations of the free ones. If one understands the free operator well, there is a temptation to consider the periodic potential as a perturbation term, at least at high energies $\lambda \rightarrow \infty$. This happens to be a highly valuable approach in many instances (e.g., in establishing the finiteness of the number of spectral gaps and various spectral asymptotics, topics addressed later in this text). However, it is also challenging and technically difficult. Even moderately comprehensive exposition alone might take a book (and in fact such books have been written [213,389]). Thus we will just indicate some of the difficulties and send the reader to the original texts for the details of various techniques employed.

It has been noticed (e.g., [137, 138, 157, 158) that the Floquet eigenvalues $\lambda_{j}(k)$ can be split into the sets of nice stable and much more complicated unstable or resonant ones. The perturbation approach works more easily in the stable region and requires a lot of ingenuity when working with the unstable one (in particular, showing that the resonant values are sufficiently rare). The reader is directed to [213, 323] and the references therein for technical details and further references.

Looking back at Theorem 5.11, one can expect the difficulties growing with the dimension, which indeed does happen. It is also much harder to handle the higher-order perturbations, e.g., periodic magnetic potential terms of Schrödinger operator. Again, the paper 323 is a good reference to look into for contemporary results and techniques.

5.4. Born-Karman approximation. A very useful way of obtaining the dispersion relation (and thus the spectrum) of a periodic operator is due to Born and Von Karman (see, e.g., [1]).

We will assume, as before, that $\Gamma=\mathbb{Z}^{n}$ (although the approach works for any lattice).

Theorem 5.12. Consider for an integer $N$ the cube $Q_{N}:=[-N, N]^{n} \in \mathbb{R}^{n}$ and define the operator $H^{(N)}$ acting as the differential expression of $H$ on $Q_{N}$ with periodic boundary conditions (i.e., on the cube $Q_{N}$ folded onto a large torus). Then

$$
\sigma(H)=\lim _{N \rightarrow \infty} \sigma\left(H^{(N)}\right)=\overline{\bigcup_{N \in \mathbb{Z}^{+}} \sigma\left(H^{(N)}\right)},
$$

where the limit is taken with respect to the Hausdorff distance on any bounded part of the real axis.

Indeed, let us denote by $\mathbb{Q B}$ the subset of the Brillouin zone consisting of all quasimomenta with components that are rational multiples of $\pi$. Then, it is a rather straightforward exercise to see that

$$
\bigcup_{N \in \mathbb{Z}^{+}} \sigma\left(H^{(N)}\right)=\bigcup_{k \in \mathbb{Q B}} \sigma(H(k)) .
$$

Now, using Lemma 5.6, one proves Theorem 5.12. 
Remark 5.13. Another important observation is that this theorem is more than just the claim about Hausdorff convergence of spectra, or density of states convergence. Indeed, such claims hold also for other reasonable choices of boundary conditions (e.g., Dirichlet), or other choices of large domains instead of large cubes assembled from the fundamental unit cubes [352. In those cases, however, one can often see spurious spectra appearing outside $\sigma(H)$, but disappearing in the limit. In the Born-Karman case, though, this does not happen, since $\sigma\left(H^{(N)}\right) \subset \sigma(H)$ for any $N$.

5.5. Analytic properties of the dispersion relation $B_{H}$. We will now explore analytic properties of the dispersion relation of the operator $H$. Consider the complex Bloch variety

$$
B_{H, \mathbb{C}} \subset \mathbb{C}^{n+1} .
$$

Theorem 5.14 ([240, Lemma 8 of $\S 4]$, [244, Theorem 4.4.2], see also [229]).

(1) There exists an entire function $f(k, \lambda)$ on $\mathbb{C}^{n+1}$, such that

(a)

$$
|f(k, \lambda)| \leq C_{p} e^{(|k|+|\lambda|)^{p}}
$$

for any $p>n$. (A similar statement holds for more general elliptic periodic operators, with the exponent $p$ depending on the dimension and order of the operator 244.)

(b)

$$
B_{H, \mathbb{C}}=\left\{(k, \lambda) \in \mathbb{C}^{n+1} \mid f(k, \lambda)=0\right\} .
$$

(2) If we only allow $p>n+1$ as the exponential order, one can make sure that $f$ is $\Gamma^{*}$-periodic w.r.t. $k$.

In particular, $B_{H, \mathbb{C}}$ is a $\Gamma^{*}$-periodic complex analytic subvariety of $\mathbb{C}^{n+1}$ of codimension 1 .

An easier statement, without estimates on growth of the function $f$, can be obtained from the analytic Fredholm theory (e.g., [244, 394, 404]). The proof of the theorem as stated uses the theory of regularized determinants in Shatten-von Neumann classes, presented in, e.g., [114, 175, 355].

5.6. Floquet variety. It is natural to reduce $B_{H}$ with respect to its $\Gamma^{*}$-periodicity (i.e., using Floquet multipliers $z=e^{i k}$ instead of crystal momenta $k$ ). We thus introduce the following (not commonly adopted) notion:

Definition 5.15. The Floquet variety $\mathcal{F}_{H}$ of $H$ is defined as follows:

$$
\mathcal{F}_{H}:=\left\{(z, \lambda) \in\left(\mathbb{C}^{*}\right)^{n} \times \mathbb{C} \mid z=e^{i k}=\left(e^{i k_{1}}, \ldots, e^{i k_{n}}\right) \text {, with }(k, \lambda) \in B_{H, \mathbb{C}}\right\}
$$

Here $\mathbb{C}^{*}$ is the punctured complex plane $\mathbb{C}^{*}:=\mathbb{C} \backslash\{0\}$.

In other words, $(z, \lambda) \in \mathcal{F}_{H}$, iff there exists a nonzero Floquet solution of $H u=$ $\lambda u$ with the Floquet multiplier $z$. 
5.7. Nonalgebraicity. As we have seen already in the free case, $B_{H}$ is the union of $\Gamma^{*}$-shifts of a single paraboloid. Thus, we get

Proposition 5.16. In the free case,

(1) All irreducible components of the Bloch variety $B_{H}$ are algebraic (shifted paraboloids). In particular, the Bloch variety $B_{H}$ is irreducible modulo $\Gamma^{*}$ shifts.

(2) The Floquet variety $\mathcal{F}_{H}$ is irreducible.

It is thus interesting to ask what to expect in terms of algebraicity and irreducibility in the nonfree periodic case.

In general, the irreducible components of neither Bloch nor Floquet variety are algebraic [139. There exist, however, examples of non-self-adjoint periodic operators $H$ with algebraic components of $B_{H}$ (see [239, Theorem 11] and [244, Theorem 4.1.2]).

In discrete problems, the Floquet varieties are algebraic [27, 243, since the corresponding operators $H(z)$ are finite Laurent series with respect to $z$ :

$$
\sum_{j \in \mathbb{Z}^{n}} H_{j} z^{j}
$$

where the sum is finite and $H_{j}$ are matrices of a finite size.

An impressive detailed study of the structure the Bloch variety for discrete periodic Schrödinger operators on $\mathbb{Z}^{2}$ was done in [172, 173 .

Another important question is about irreducibility of the Bloch variety in higher dimensions, which is addressed in the next subsection.

5.8. Irreducibility. It is conjectured that the analog of the statement (2) of Proposition 5.16 holds in higher dimensions:

Conjecture 5.17. The Bloch variety of the self-adjoint periodic Schrödinger operator $H=-\Delta+V(x)$ is irreducible modulo $\Gamma^{*}$.

A stronger version conjectures the same for more general periodic self-adjoint second-order elliptic operators.

Proving this conjecture seems to be extremely hard. It has been proven in dimension 2 by a tour de force in the paper 229 , as well as in the 2D discrete case in 172,173 .

A much weaker conjecture formulated below is already nontrivial:

Conjecture 5.18. The Bloch variety $B_{H}$ for a periodic elliptic second-order operator $H$ with sufficiently decent coefficients does not have any flat components $\lambda=$ const.

We will see later on, how important is the resolution of this conjecture for spectral theory. That is why it has attracted the attention of many mathematicians and has been proven in many cases (albeit still not in its full generality); see Section 6.3.

We provide here an easy to complete sketch of the proof of the following wellknown result, due to Thomas [382, although he did not formulate the statement in this form (see also [332, Section VIII.16]):

Theorem 5.19 (382]). Let $V \in L_{\infty}\left(\mathbb{R}^{n}\right)$ be periodic. Then the Bloch variety of the Schrödinger operator $-\Delta+V(x)$ has no flat components $\lambda=\lambda_{0}$. (Notice that it is not assumed that the potential $V$ is real.) 
Proof. Suppose that this is incorrect, i.e., there exists $\lambda_{0} \in \mathbb{C}$ such that the equation $-\Delta(k) u+V u=\lambda_{0} u$ has a nontrivial 1-periodic solution $u$ for any $k \in \mathbb{C}^{n}$. Absorbing the spectral parameter into the potential, we can assume that $\lambda_{0}=0$. Thus, we conclude that

$$
\Delta(k) u=V u \text { has a nontrivial solution } u \text { for any } k \in \mathbb{C}^{n} .
$$

Let $M:=\|V\|_{L_{\infty}}$. Then $\|V u\|_{L^{2}(\mathbb{T})} \leq M\|u\|_{L^{2}(\mathbb{T})}$ and thus

$$
\|\Delta(k) u\|_{L^{2}(\mathbb{T})} \leq M\|u\|_{L^{2}(\mathbb{T})}
$$

for any $k$ (with a nontrivial $u$ depending on $k$ ).

Let us now pick quasimomentum with a large imaginary part and judiciously chosen real part. Namely, assuming that $\Gamma=\mathbb{Z}^{n}$ (analogous consideration works for any lattice), let

$$
k=(\pi+i 2 a, 0, \ldots, 0), a \in \mathbb{R} .
$$

Expansion of the periodic function $u$ into a Fourier series easily leads to the estimate

$$
\|\Delta(k) u\|_{L^{2}(\mathbb{T})} \geq|a|\|u\|_{L^{2}(\mathbb{T})} .
$$

If $a>M$, we get a contradiction, unless $\|u\|=0$, which we assumed does not happen.

The assumption of boundedness of the potential is way too strong (see, e.g., [332, Section VIII.16]).

In fact, a stronger statement holds:

Theorem 5.20. Under the same conditions as in Theorem [5.19, for any $\lambda_{0} \in \mathbb{R}$, the level set of the dispersion relation (isoenergy surface, or Fermi surface, see Section 5.11)

$$
F_{\lambda_{0}}=\left\{k \in \mathbb{R}^{n} \mid H(k) u=\lambda_{0} u \text { has a nonzero solution }\right\}
$$

has measure zero in $\mathbb{R}^{n}$.

We will see later that this leads to the absolute continuity of the spectra of periodic Schrödinger operators, which was the main goal in 382. Due to this relation, the statements of Theorems 5.19 and 5.20 have been extended in the last three decades to a large variety of scalar and matrix periodic operators of mathematical physics (e.g., Pauli, Dirac, magnetic Schrödinger, Maxwell, and elasticity operators, see Section 6.3 and references therein), although the proof of the full Conjecture 5.18 remains elusive.

Remark 5.21.

(1) The consideration of the proof of Theorem 5.19 can be carried over to a variety of (probably not too interesting) periodic operators $L(D)+V(x)$, where $L(D)$ is a constant coefficient operator; see [239, p. 28] and the references therein.

(2) An often overlooked interesting observation was made in [115, 116] that the Fermi surface contains not only no flat parts but also no open pieces of some nonflat rational curves.

We need first the following auxiliary statement:

Lemma 5.22. Let $a(k)$ be a real analytic function on (a connected open domain of) $\mathbb{R}^{n}$. If the measure of the zero set of a is positive, then the function vanishes identically. 
Remark 5.23. This claim has several elementary proofs, but for some reason it is hard to find it even stated in most texts on real analytic geometry ([236] is an exception). Due to article size limitations, let me just indicate three approaches, each of which can be easily completed by a graduate student: using Fubini's theorem (as suggested in [25, Corollary 5.2]); by induction with respect to the dimension (suggested in [236, begginning of Section 4.1]); by covering the set with a countable set of smooth submanifolds, obtained using implicit function theorem [293]. It can also be derived from the Weierstrass Preparatory Theorem (e.g., [189]). It is also a direct corollary of the Lojasiewicz's result on existence of Whitney stratification of real analytic sets 271,272, as well as of the desingularization theorems by Bierstone and Milman [31,32, which seem to be quite an overkill in both cases. Another nontrivial derivation can be found in Federer's book [131, Section 3.1.24, p. 240].

Now Theorem 5.20 becomes a simple corollary of Theorem 5.19 . Indeed, the level set of the dispersion relation is the set of real zeros of an entire function $f\left(k, \lambda_{0}\right)$ (see Theorem 5.14 for its definition). Thus, if this set has positive measure, then, according to Lemma 5.22 the function vanishes identically, and thus $f\left(k, \lambda_{0}\right)=0$ for all $k \in \mathbb{C}^{n}$. This is impossible according to Theorem 5.19 ,

One should notice that for periodic elliptic operators of orders higher than 2 neither of these conjectures holds true; e.g., there are examples of such operators of the fourth order whose Bloch variety is reducible and, moreover, has a flat irreducible component [244, pp. 135-136].

Let us address another issue related to irreducibility. We have seen that in the free case any nonzero $\Gamma^{*}$-shift of an irreducible component $\Sigma$ of $B_{H}$ produces another component, different from $\Sigma$. In other words, irreducible components are not invariant with respect to any nonzero $\Gamma^{*}$-shifts. It seems that in the presence of a generic periodic potential the situation is different.

Conjecture 5.24. For a generic potential, unless an irreducible component $\Sigma \subset$ $B_{H}$ is algebraic, there exists a nonzero $\Gamma^{*}$-shift that leaves it invariant.

If this is correct, then perturbation of the free operator by a periodic potential not only deforms the paraboloids, but also glues them together.

5.9. Extrema. Since, according to (5.5) - (5.7) the edges of the spectrum occur at extrema of the band functions, studying these extrema is an important task.

5.9.1. Location. In $1 \mathrm{D}$, the extrema of the dispersion relation $\lambda(k)$ occur only at $k=0$ and $k=\pi$ in the Brillouin zone, due to the monotonicity (Lemma 1.16) of band functions on the reduced Brillouin zone.

Let us try to look at the points $k=0$ and $k=\pi$ in a different light. The reciprocal lattice $\Gamma^{*}$ is invariant with respect to the symmetry group generated by its shifts and "time reversion" $k \mapsto-k$. One discovers then that the points $k=0, \pi$ are exactly the fixed points of some of these symmetries inside of the reduced Brillouin zone.

In higher dimensions, the symmetry group can be larger [51]. If one looks at the free case, one discovers that critical points of these overlapping paraboloids do occur at some symmetry points as well. Most computations for periodic Schrödinger and Maxwell operators also showed this effect. This has led to the following:

Formerly popular belief. For $-\Delta+V(x)$, the extrema of dispersion relation are attained at fixed points $k \in \mathcal{B}$ of some symmetries (probably at the highest symmetry 
points); i.e., computing the dispersion only around the symmetry points gives the correct spectrum as a set.

No monotonicity (or any other) reason for this claim exists. Some numerical evidence against it has been around, but is not widely known and/or believed. This claim was discussed and disproved analytically in [130, 190] (see also simpler waveguide examples in papers [49,50] and a physics paper [82]). It is also discussed in 190 why in practice the extrema are indeed often located at the symmetry points.

5.9.2. Generic structure of spectral edges. After discussing the possible locations of the extrema, we move to the probably more important question of their structure. The following complications can (and do) occur at a spectral edge value $\lambda=c$ :

A: The extremal value $c$ is attained by more than one band function.

B: The extremum $c$ of a single band function $\lambda_{j}$ is nonisolated (i.e., a "Mexican hat" type picture occurs).

C: The extremum is isolated, but degenerate (i.e., the Hessian of $\lambda_{j}(k)$ at the extremum point is degenerate).

Although all of the above can occur (e.g., [146, 349]), the general belief is that generically they do not (see, e.g., 71]).

Conjecture 5.25. Generically (with respect to the potentials and other free parameters of the operator), the extrema of band functions

(1) are attained by a single band;

(2) are isolated;

(3) are nondegenerate, i.e., have nondegenerate Hessians.

In other words, one conjectures that generically near a spectral edge the dispersion relation looks like a single parabolic shape, i.e., it resembles the dispersion relation at the bottom of the spectrum of $-\Delta$. As we will see later (Section 7), then various analogs of the properties of the Laplacian would be applicable to a generic periodic elliptic operator.

Why would one conjecture this? Well, existence of a degenerate extremum of the dispersion relation is an analytic equality type restriction. Then it is natural to think that it should either hold almost never, or for (almost) all operators. It is hard to believe that such a restriction for all periodic potentials exists.

The common idea is that generically, the dispersion relation probably behaves like the spectrum of a "generic" family of self-adjoint matrices. This has been conjectured in various (explicit or implicit) forms by several authors; see [16, 71, 304,305).

Although the validity of conditions (1)-(3) of Conjecture 5.25] is often assumed in mathematics and physics literature (it is involved with the definition of effective masses in solid state physics, homogenization, Green's function asymptotics, Liouville type theorems, impurity spectra in lacunas, Anderson localization, etc., see Section 7), the conjecture remains largely unproven.

Let us mention what is known in this regard.

The following classical result of [224] establishes the conjecture (in a stronger form) at the bottom of the spectrum of a Schrödinger operator with periodic electric potential. 
Theorem 5.26 ([224]). Let

$$
H=-\Delta+V(x)
$$

be a periodic Schrödinger operator in $\mathbb{R}^{n}$. Then the bottom of the spectrum of $H$ is attained by the nondegenerate minimum at $k=0$ of the lowest eigenfunction $\lambda_{1}$ only.

An analogous result was obtained for the periodic 2D Pauli operator in 45. An improvement to Theorem 5.26 under an additional symmetry assumption on the potential, was obtained in 193. Regretfully, the arguments of [45, 224] are not applicable in the case of an internal spectral edge.

The conjecture was not only stated, but also proven for "small" potentials in 2D 71]:

Theorem 5.27 (71]). For given lattice $\Gamma \subset \mathbb{R}^{2}$ and natural number $N$, there exists a residual set $R$ in a neighborhood of zero in $C^{\infty}(\mathbb{T}, \mathbb{R})$, such that for any potential $V \in R$ and all $j \leq N$, the Floquet eigenvalues $\lambda_{j}(k)$ are simple and $\lambda_{j}(k)$ is a Morse function on the torus $\mathbb{T}$.

The following partial result was proven in 228]:

Theorem 5.28 ([228]). Claim (1) of Conjecture 5.25 holds for a generic Schrödinger operator with periodic electric potential.

The second claim of the conjecture was recently proven for Schrödinger operators in $2 \mathrm{D}$ in 146 .

It was shown in 349,350 , that the presence of a magnetic potential can create degeneracies even at the bottom of the spectrum.

The conjecture makes sense also in the discrete case and was proven for periodic Schrödinger operators on $\mathbb{Z}^{2}$ (see the overview 172 for the results and exact references).

For more general $Z^{2}$-periodic graphs there is the following partial result:

Proposition 5.29 ([109]). Let $H$ be the periodic Laplace-Beltrami type operator 12 on a $\mathbb{Z}^{2}$-periodic graph $\Gamma$, having just two vertices (atoms) per a fundamental domain, with all possible edges between adjacent copies of fundamental domain being allowed. Then the set of parameters (vertex and edge weights) for which the dispersion relation has a degenerate extremum is a (semi-)algebraic subset of codimension 1 in the space of all parameters.

However, as an example [146] of a stable nonisolated extremum shows, one has to be careful, allowing for a sufficiently large space of perturbation parameters (analogs of potentials).

In principle, if the conjecture were proven for the discrete case, one could attempt to bootstrap the result to the continuous case (such a procedure was used for a different purpose in [190]). A direct approach to the continuous case, skipping the discrete one, would be desirable.

Allowing change of the lattice of periods to a sublattice, possibility of removing degenerate edges by perturbation of the potential was shown in 2D in 324.

\footnotetext{
${ }^{12}$ See $27 \sqrt[67]{67}$ for the explicit form of such operators.
} 


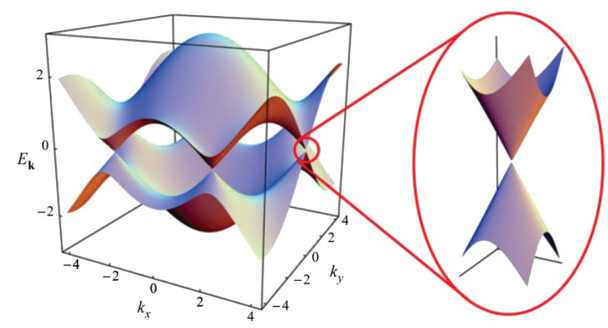

Figure 13. Dirac cones for the tight-binding model of graphene.

5.10. Dirac cones. When two branches of the dispersion relation meet, they often form a conical junction point, called a Dirac cone or sometimes a diabolic point 29 . The former name reflects the fact that the conical structure resembles the one for the dispersion of a 2D massless Dirac equation 218.

Such conical structures are usually unstable under perturbation of parameters (e.g., the potential) of the operator. However, it has been noticed that if the structure has honeycomb lattice symmetry, preserved under perturbation, this protects the cone from splitting. Thus, in particular, such a cone mandatorily arises in the dispersion relation of the famous graphene, which explains its amazing electric properties (see, e.g., 218).

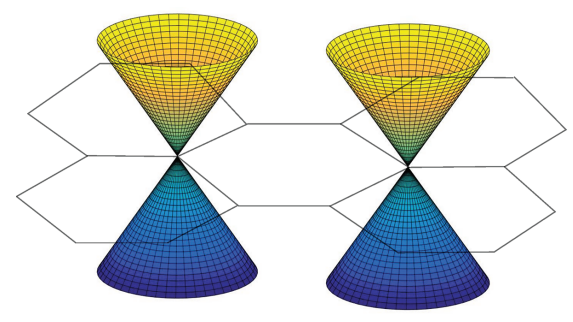

FiguRE 14. Dirac cones for the honeycomb structure.

The observation of the mandatory appearance of Dirac cones in honeycombsymmetric structures was first made (way before the graphene came into play) for the tight-binding model of the discrete honeycomb lattice 392], which provides an approximate picture of the graphene's first two dispersion bands. An infiniteband quantum graph model, which has an infinite-dimensional freedom of choosing honeycomb-symmetric potentials was considered in 255, where the detailed structure of the spectrum and dispersion relation, including in particular the presence of Dirac cones, was described 13 For 2D Schrödinger operators with honeycombsymmetric potentials, existence and stability of Dirac cones was established, explained, and studied [26, 132, 136, 186. The Schrödinger operator with honeycomb lattice of point scatterers was considered in 263. Carbon nanotubes as folded sheets of graphene have been studied in various papers, see, e.g., [106, 234, 255] and references therein.

${ }^{13}$ The article 255 also contained the detailed spectral analysis of quantum graph models of all possible types of carbon nanotubes. 
One can say, however, that the story does not end here, since the presence of stable Dirac cones (including very interesting nonisotropic ones) has been observed for more general 2D (graphyne) structures, which lack honeycomb symmetry; see, e.g., [122 for physics discussion and [106, 107] for quantum graph studies of a simplest graphyne. So far, there is no complete understanding of this phenomenon (analogous to the one provided in [26, 135, 186] for the honeycomb case).

5.11. Fermi surfaces. The notion of the Fermi surface is among the central ones in solid state theory as one can see from any solid state textbook, e.g., [11,63, 225, 409. There are plenty of books and databases dedicated to the Fermi surfaces of various crystals, e.g., 80, 81, 140, 191,408, 410. The reason is that many physical properties of, e.g., metals depend upon the geometry of its Fermi surface. We will try to show that questions about the analytic geometry of Fermi surfaces are intimately related to some basic spectral and other properties of the relevant operator. Answering these questions is usually extremely hard (see, e.g., 173] and a shorter survey [172] devoted solely to the case of a discrete periodic Schrödinger operator on $\mathbb{Z}^{2}$ ).

To define a Fermi surface, let us consider the (real or complex) dispersion relation $B_{H}$ as the graph of the multiple valued function

$$
k \in \mathbb{C}^{n} \mapsto \sigma(H(k)) .
$$

Definition 5.30. The Fermi surface ${ }^{14} F_{\lambda, H}$ of $H$ at a scalar value $\lambda$ is the $\lambda$-level set of the dispersion relation. I.e.,

Complex Fermi surface is:

$$
\begin{aligned}
F_{\lambda, H, \mathbb{C}}: & =\left\{k \in \mathbb{C}^{n} \mid(k, \lambda) \in B_{H, \mathbb{C}}\right\} \\
& =\left\{k \in \mathbb{C}^{n} \mid H(k) u=\lambda u \text { has a nonzero solution }\right\} .
\end{aligned}
$$

Real Fermi surface for a real $\lambda$ is:

$$
\begin{aligned}
F_{\lambda, H}: & =\left\{k \in \mathbb{R}^{n} \mid(k, \lambda) \in B_{H}\right\} \\
& =\left\{k \in \mathbb{R}^{n} \mid H(k) u=\lambda u \text { has a nonzero solution }\right\} .
\end{aligned}
$$

An obvious statement is:

Lemma 5.31. The Fermi surface is $\Gamma^{*}$-periodic.

The following result follows from its analog for the Bloch variety $B_{H}$ :

Theorem 5.32. $F_{\lambda, H}$ is the zero set of an entire function $f(k)$ of the same exponential order as for $B_{H}$ (see Theorem [5.14).

The following result, which holds in a very general setting of elliptic periodic operators (compare with Theorem 5.20 ) is useful:

Theorem 5.33. At any energy level $\lambda \in \mathbb{R}$, the real Fermi surface either has measure zero in $\mathbb{R}^{n}$, or it coincides with the whole $\mathbb{R}^{n}$ (and thus the complex Fermi surface at this level is $\mathbb{C}^{n}$ ).

\footnotetext{
${ }^{14}$ Also called isoenergy surface.
} 


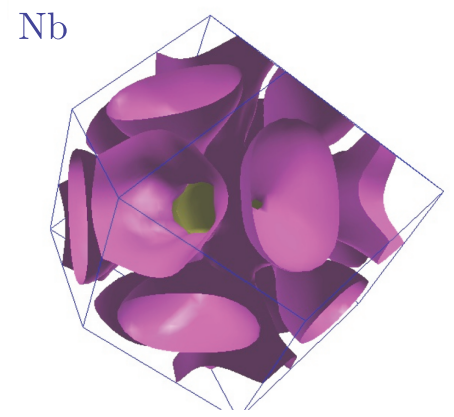

Figure 15. The Fermi surface of Niobium (Nb). The picture is borrowed from the URL [140, where one can find many more (often more complex) pictures of Fermi surfaces.

\section{Remark 5.34.}

(1) In physics, the name Fermi surface is used only for a specific real level of the energy $\lambda$, the so-called Fermi level $\lambda_{F}$ [11,63, 225, 409. It is convenient for us, though, to consider Fermi surfaces at arbitrary levels.

(2) The definition of the Fermi surface seems to be rather innocuous. Just to impress upon the reader its possible complexity, Figure 15 presents the Fermi surface of Niobium (Nb).

Conjecture 5.35. The Fermi surface for $H=-\Delta+V(x)$ is irreducible (modulo $\left.\Gamma^{*}\right)$ for $\lambda \in \mathbb{R}$, possibly except for a discrete set of values of $\lambda$.

This conjecture has been proven for discrete Schrödinger operator on $\mathbb{Z}^{2}$ [172, 173 . and in the case of a continuous Schrödinger operator with periodic electric potential in dimensions $n=2,3$ and separable potential $V(x)=\sum V_{j}\left(x_{j}\right)$, as well as in 3D for potential $U\left(x_{1}, x_{2}\right)+V\left(x_{3}\right)$, where the axis $x_{j}$ are oriented along the basis vectors of $\Gamma[258$.

As we will see in Section 6.6 the "esoteric" question of irreducibility of the Fermi surface is closely related to the question of absence of impurity eigenvalues embedded into the continuous spectrum.

5.12. Bloch bundles. Let $S \subset \sigma(H)$ be a subset consisting of $m$ spectral bands and surrounded by spectral gaps. We will call such a subset a composite band (see Figure 16). Let us surround $S$ with a contour $\Gamma$ (Figure 16) and introduce the corresponding $m$-dimensional spectral projector for $H(k)$

$$
P(k):=\frac{1}{2 \pi i} \oint_{\Gamma}(\zeta-H(k))^{-1} d \zeta .
$$

The projector $P(k)$ depends analytically on $k$ in a complex neighborhood of $\mathbb{T}^{*}$ in $\mathbb{C}^{n}$. Its range forms the so-called Bloch bundle over this neighborhood, which corresponds to the composite band $S$. Sections of this bundle form the invariant subspace for $H$ that corresponds to $S \subset \sigma(H)$.

This bundle over the torus $\mathbb{T}^{*}$ can be topologically nontrivial (e.g., in the presence of a magnetic potential (Thouless [383]) or in the case of topological insulators [28]).

As we have seen, the bundle is defined and analytic in a complex neighborhood of the torus. We are sometimes interested in its analytic triviality. The question 


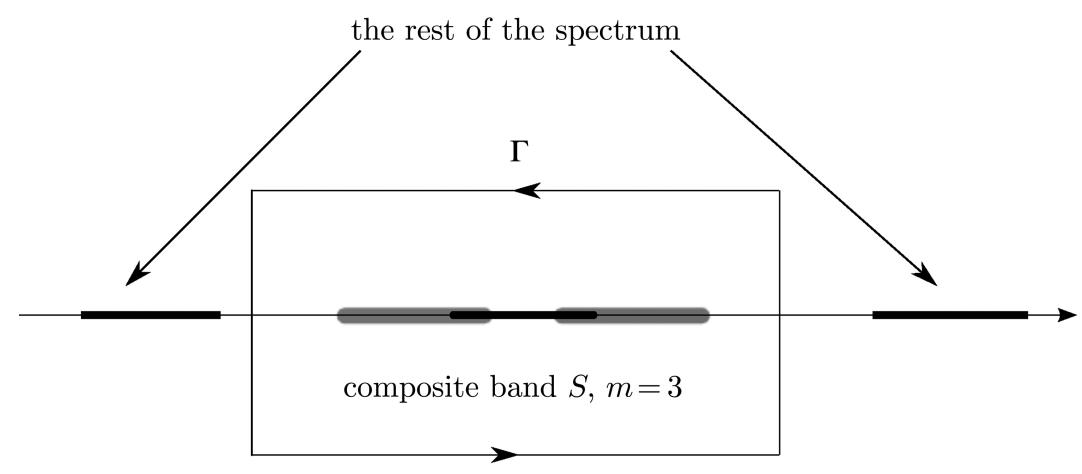

Figure 16. A composite band composed of three spectral bands.

arises whether there are additional analytic obstructions to this. The following result is a simple incarnation of the so-called Oka's principle [180, 189]:

Theorem 5.36 (e.g., 249]). Topological triviality of the Bloch bundle over $\mathbb{T}^{*}$ is equivalent to its analytic triviality in a complex neighborhood.

Indeed, there is a neighborhood of the torus that is the product of $1 \mathrm{D}$ complex domains. Then it is a Stein manifold [180, and the above result is a part of the famous Grauert's theorem [179] for such manifolds.

A variety of results on sufficient conditions of triviality of Bloch bundles is known; see, e.g., 249, 313] and references therein (see also 404 for the survey of techniques of Banach bundles).

5.13. Analyticity in the space of parameters. An often very useful thing to do is to add to the spectral parameter $\lambda$ and quasimomenta $k$ an infinite-dimensional space $P$ of parameters of the periodic operator (i.e., an appropriate Banach space of periodic electric and/or magnetic potentials). Then the Fermi and Bloch varieties can be defined in this extended space. When the space of parameters is defined properly, it is usually straightforward to prove the following:

Meta-Theorem 5.37. Bloch and Fermi surfaces are analytic subsets in the enlarged space $\mathbb{C}^{n+1} \times P\left(\right.$ respectively $\left.\mathbb{C}^{n} \times P\right)$.

5.14. Inverse problems. Inverse spectral problems are known to arise frequently and have been much studied for periodic ODEs (e.g., [1, 113, 163, 164, 262, 282, 307. and references therein).

The periodic PDEs in this regard are much trickier, and there are not that many results available. We look here at a few examples.

5.14.1. Borg's theorem. The famous Borg's uniqueness theorem for the Hill operator $-\frac{d^{2}}{d x^{2}}+q(x)$ with periodic potential $q$ says:

Theorem 5.38 (48]). The following claims are equivalent:

(1) The potential is constant.

(2) There are no spectral gaps.

(3) There exists an entire function, whose graph $\lambda=f(k)$ belongs to the dispersion relation $([16])$. 
The equivalence $(1) \Leftrightarrow(2)$ fails miserably in dimensions $n>1$ ! In fact, as we will see later, for any sufficiently small bounded periodic potential $V(x)$ in $\mathbb{R}^{2}$ and $\mathbb{R}^{3}$, the Schrödinger operator $-\Delta+V(x)$ has no gaps in the spectrum. Moreover, when the gaps do appear, there are only finitely many of them. Thus, they clearly carry insufficient information for recovery of the potential. Spectral gaps in higher dimensions do not provide nearly as much information as in the ODE case. However, the equivalence $(1) \Leftrightarrow(3)$ probably still holds:

Conjecture 5.39. The following claims are equivalent:

(1) The potential is constant.

(2) There exists an entire function, whose graph $\lambda=f(k)$ belongs to the dispersion relation.

In $2 \mathrm{D}$, the conjecture was proven in 229 .

5.14.2. Floquet rigidity. Let us consider the Schrödinger operator $H=-\Delta+V(x)$ with the potential periodic with respect to a lattice $\Gamma$ and the corresponding Bloch operators $H(k)$, which have, as we know, discrete spectra.

Definition 5.40.

- The periodic spectrum is defined as follows:

$$
\sigma_{0}(H):=\sigma(H(0))=\text { spectrum of } H \text { on } \Gamma \text {-periodic functions. }
$$

- The $k$-spectrum is defined as follows:

$$
\begin{aligned}
\sigma_{k}(H): & =\sigma(H(k)) \\
& =\text { spectrum on Bloch functions with quasimomentum } k .
\end{aligned}
$$

Clearly, the union over all $k$ of the graphs of these spectra forms the Bloch variety for $H$.

Definition 5.41.

- The $\Gamma$-periodic potentials $V$ and $W$ are isospectral if

$$
\sigma_{0}(-\Delta+V)=\sigma_{0}(-\Delta+W) .
$$

- The $\Gamma$-periodic potentials $V$ and $W$ are Floquet isospectral if

$$
\sigma_{k}(-\Delta+V)=\sigma_{k}(-\Delta+W), \forall k \in \mathbb{R}^{n} .
$$

One is interested in understanding when two potentials are isospectral, or even Floquet isospectral. These questions have been understood well in 1D (see, e.g., 282 ), while for $n \geq 2$, the problems are far from being resolved.

There are simple examples of isospectral potentials. For instance, one observes:

\section{Lemma 5.42.}

(1) Potentials $V(x)$ and $W(x)=V( \pm x+a)$, for any $a \in \mathbb{R}^{n}$, are Floquet isospectral.

(2) If the lattice $\Gamma$ is invariant with respect to an orthogonal transformation $O$ of $\mathbb{R}^{n}$, then the potentials $V(x)$ and $V(O x)$ are isospectral.

Imposing the following condition on the lattice $\Gamma$, one eliminates the second option in the lemma above: 
Definition 5.43. A lattice $\Gamma$ has a simple length spectrum, if the following holds:

$$
\text { if } \gamma_{1}, \gamma_{2} \in \Gamma \text { and }\left|\gamma_{1}\right|=\left|\gamma_{2}\right| \text {, then } \gamma_{2}= \pm \gamma_{1} \text {. }
$$

One other option is to use the well known results on 1D isospectrality. Indeed, let $v(s)$ and $w(s)$ be smooth 1-periodic functions of one variable, and let $\delta$ be a vector such that

$$
\{\delta \cdot \gamma \mid \gamma \in \Gamma\}=\mathbb{Z}
$$

It is easy to establish [125, 127] that if $1 D$ potentials $v$ and $w$ are isospectral, then the "ridge" $n$-dimensional potentials $v(\delta \cdot x)$ and $w(\delta \cdot x)$ are isospectral. One wonders whether there are any other options for isospectrality (Floquet isospectrality) 15

Many significant results on multidimensional isospectrality were obtained in [125, 127. (see also an overview in 126]), mostly under conditions of analyticity of the potentials and the simplicity of the length spectrum (5.25). See also preceding and following works of various authors with additional results and related discussions in 21, 123 125, 176, 178, 187, 188, 209, 211, 386, 393.

We present below a few results of $125,127,16$

Theorem 5.44. Suppose that the length spectrum of $\Gamma$ is simple and $\Gamma$-periodic potentials $V$ and $W$ are analytic, then

- If $V$ and $W$ are isospectral, then they are Floquet isospectral.

- Let $k_{0} \in \mathbb{R}^{n}$ be such that $\cos \left(2 \pi k_{0} \cdot \gamma\right) \neq 0$ for all $\gamma \in \Gamma$. If $\sigma_{k_{0}}(-\Delta+V)=$ $\sigma_{k_{0}}(-\Delta+W)$, then $V$ and $W$ are Floquet isospectral.

This result shows that (under appropriate conditions) the whole Floquet spectrum (i.e., the whole Bloch variety) has overdetermined information, and the fiber of $B_{H}$ over an appropriate quasimomentum $k_{0}$ carries as much information as the whole $B_{H}$.

It is natural to look in the horizontal, i.e., along quasimomenta, direction. In particular,

Does the knowledge of a single band function $\lambda_{j}(k)$ determine the whole dispersion relation (Bloch variety)?

In fact, the Conjecture 5.17 (proven in 1D and 2D) implies, if correct, that even any open part of a single branch $\lambda_{j}(k)$ uniquely determines the dispersion relation.

Now let $V$ be a $C^{\infty} \Gamma$-periodic potential with the Fourier series expansion

$$
V(x)=\sum_{\delta \in \Gamma^{*}} a_{\delta} e^{i \delta \cdot x} .
$$

Definition 5.45. Let $\gamma \in \Gamma$. The reduced potential $V_{\gamma}(x)$ is defined as follows:

$$
V_{\gamma}(x)=\int_{0}^{1} V(x+s \gamma) d s=\sum_{\delta \cdot \gamma=0} a_{\delta} e^{i \delta \cdot x} .
$$

\footnotetext{
${ }^{15}$ One can easily achieve Floquet isospectrality by allowing complex potentials [244, Theorem 4.1.2].

${ }^{16}$ The sophisticated techniques used in [125,127 to prove the results involved, in particular, the spectral invariants extracted from the heat and wave kernel asymptotics.
} 
Another important observation in [125 127] concerns the relations between the Floquet spectra of the original and reduced potentials:

Theorem 5.46. For any $\gamma \in \Gamma$, the Floquet spectrum $\sigma_{k}(-\Delta+V)$ determines uniquely $\sigma_{k}\left(-\Delta+V_{\gamma}\right)$.

Now let $\delta \in \Gamma^{*}$ be such that there exists $\gamma_{0} \in \Gamma$ for which $\delta \cdot \gamma_{0}=2 \pi$. Then one can introduce the $1 \mathrm{D}$ directional potential

$$
V_{\delta}(s):=\sum_{n=-\infty}^{\infty} a_{n \delta} e^{i n s} .
$$

Theorem 5.47. The Floquet spectrum $\sigma_{k}(-\Delta+V)$ determines uniquely the Floquet spectra of the $1 D$ spectral problems

$$
\left(-|\delta|^{2} \frac{d^{2}}{d s^{2}}+V_{\delta}(s)\right) u(s)=\lambda u(s)
$$

for any directional potential $V_{\delta}$.

The isospectrality of potentials $V( \pm x+a)$ suggests a question whether under some conditions one can prove that, modulo shifts, the number of isospectrality classes is finite. A variety of important results of this type can be found in the paper [127. See also [172, 173] for inverse spectral results for discrete periodic operators on $\mathbb{Z}^{2}$.

An alternative approach to the inverse spectral problem for periodic potentials was provided in [389, Ch. 4].

\section{Spectral structure of Periodic elliptic operators}

As we have seen, both in 1D and in higher dimensions, periodic elliptic operators have band-gap structure of their spectra. There are, however, significant differences between ODE and PDE cases. We make the comparison in Table 1.

The rest of this section is devoted to discussing various spectral properties of periodic operators, such as the band-gap structure of the spectrum, the qualitative structure of the spectrum, eigenfunction expansions, etc.

6.1. Spectral gaps. We start with considerations of spectral gaps: their existence, number, and ways of creating them.

TABLE 1

\begin{tabular}{|c|c|c|}
\hline & $n=1$ & $n>1$ \\
\hline band overlaps & none & frequent \\
\hline gaps & generically all open & many gapless potentials \\
\hline no gaps & constant potential & all small potentials \\
\hline free operator bands & touch to cover $\mathbb{R}^{+}$ & overlap to cover $\mathbb{R}^{+}$ \\
\hline
\end{tabular}


6.1.1. Existence of gaps. As we have discussed already, according to Borg's theorem, in $1 \mathrm{D}$ every nonconstant periodic potential creates spectral gaps. This is manifestly incorrect in higher dimensions, e.g.:

Theorem 6.1. If $n=2,3$ and the $L_{\infty}\left(\mathbb{R}^{n}\right)$-norm of a periodic potential $V(x)$ is sufficiently small, the Schrödinger operator $H=-\Delta+V(x)$ in $L^{2}\left(\mathbb{R}^{n}\right)$ has no spectral gaps.

This is an immediate consequence of the first two claims of Theorem 5.11, if one considers $H$ as a perturbation of the free operator $-\Delta$.

6.1.2. Maximal abelian coverings and Sunada's no gap conjecture. Consider the standard covering $\mathbb{R}^{n} \mapsto \mathbb{T}\left(=\mathbb{R}^{n} / \mathbb{Z}^{n}\right)$. Its deck group is $\mathbb{Z}^{n}$, which coincides with $H_{1}(\mathbb{T}, \mathbb{Z})$. It can also be understood as the quotient of the universal cover of the torus by the commutator subgroup of the fundamental group $\pi_{1}(\mathbb{T})$.

Definition 6.2. The maximal abelian covering of a compact manifold $X$ is the covering $Y \mapsto X$ with the deck group $H_{1}(X, \mathbb{Z})$, obtained as the quotient of the universal cover of $X$ by the commutator subgroup of the fundamental group $\pi_{1}(X)$.

The following monotonicity result holds [370]:

Theorem 6.3. Let $Y \mapsto X$ be a Riemannian covering with an amenable deck group. Then

$$
\sigma\left(-\Delta_{X}\right) \subset \sigma\left(-\Delta_{Y}\right)
$$

where $\Delta_{M}$ denotes the Laplace-Beltrami operator on a Riemannian manifold $M$.

Thus, the spectrum of the Laplacian on the maximal abelian covering is the largest (and thus has the fewest gaps) among all abelian coverings of the same base. The following, still not proven, no gap conjecture has been formulated by Sunada 372,374 :

Conjecture 6.4. Let $Y \mapsto X$ be the maximal abelian covering of a compact Riemannian manifold $X$ of a constant negative curvature. Then the spectrum of the Laplace-Beltrami operator $-\Delta_{Y}$ on $Y$ has no gaps.

An analogous conjecture has been formulated in the graph case, where the base graph $X$ is assumed to be regular (i.e., degrees of all its vertices are the same). The graph version of the conjecture was proven for all regular graphs of even degree [199] and for various examples of regular graphs of odd degree [199,248, e.g., the so called $K 4$ graph $X$.

6.1.3. Number of gaps. Bethe-Sommerfeld conjecture. What about the possible number of spectral gaps, if they exist at all? In 1D, statement (3) of Theorem 1.12 shows that generically the number of gaps is infinite [224].

The situation is quite different in dimensions $n>1$, as the following old BetheSommerfeld Conjecture (BSC) [30] shows:

Conjecture 6.5. When $n>1$, there can only be a finite number of gaps in the spectrum of any periodic Schrödinger operator

$$
-\Delta+V(x)
$$

in $\mathbb{R}^{n}$. 
By now, after several decades of effort, the conjecture has been proven in its full generality. The story started with the works by Popov and Skriganov 330. and Dahlberg and Trubowitz [84] for $n=2$ and by Skriganov [359] for $n=3$. Theorem 5.11 shows that the issue should become much harder after that, and it does. For $n \geq 4$, the conjecture was established for the case of rational lattices 358, 360. For $n=4$, the conjecture was proven for arbitrary lattices by Helffer and Mohamed [194. Finally, in Parnovski's paper [317, the proof is provided in any dimension $n \geq 2$ and arbitrary lattice, under smoothness conditions on the potential (an alternative approach was offered by Veliev [387 389]). Predictably, the case of presence of magnetic potential turned out to be even harder. The result was established in the two-dimensional case by Mohamed [294] and Karpeshina 215]. A major advance both in techniques and results (allowing pseudo-differential lowerorder terms) was made by Parnovski and Sobolev in 323. (this paper also contains a nice review and bibliography of the topic). Other related results on BSC (including, e.g., Maxwell operator) can be found in [20, 121, 261, 296, 318, 321, 322, 342, 366, 391, and in the historical survey in 323 .

Remark 6.6.

(1) A stronger version of BSC for any second-order periodic elliptic operator is still not proven.

(2) The Maxwell operator case is mostly unresolved, except in a very special case considered in 391.

(3) The validity of BSC for periodic waveguide systems has not been established and is sometimes questioned.

(4) For periodic graphs/quantum graphs the statement of BSC does not hold 27.

6.1.4. Gap creation. Presence of gaps is necessary in many instances. E.g., they are responsible for properties of semi-conductors. Fortunately, such materials exist already in nature. The situation is different, for instance, when one tries to create the so-called photonic crystals, where the operator of interest is Maxwell in a periodic medium and one needs to create such a medium with spectral gaps 206, 245. We know by now that periodicity, although leading to the band-gap structure of the spectrum, does not guarantee existence of gaps. We thus address here briefly how spectral gaps can be "engineered".

Let us start with the Schrödinger operator $-\Delta+V(x)$. As we have just said, engineering gaps are not such a hot issue in this case. It also happens to be rather easy to create spectral gaps. Indeed, the following procedure can be easily made precise: Creating a local potential well, one can create a bound state (eigenvalue) below the spectrum of the Laplacian. Now, periodically repeating copies of the well at large distances from each other will lead to spreading the eigenvalue into a thin spectral band at a distance from the spectrum of the free operator, and thus it creates a periodic medium with a spectral gap.

This potential well technique fails for Maxwell and some other operators when local perturbation cannot lower the bottom of the spectrum. Another deficiency here is that the resulting gaps are rather hard to manipulate.

Another approach is creating very high contrast media, which succeeds in creating spectral gaps for periodic Maxwell operators [141 143, 145] as well as for Laplace-Beltrami operators on abelian coverings of compact Riemannian manifolds [197, and references therein; 331. A two-scale homogenization approach has 


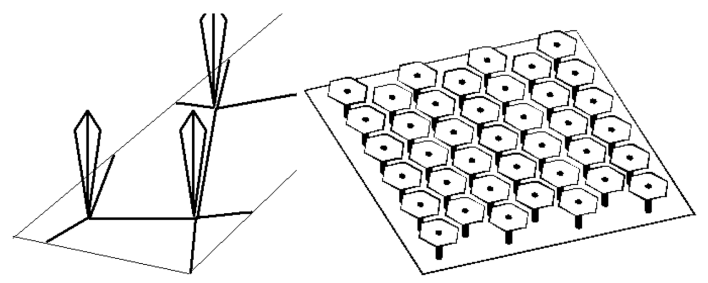

Figure 17. A decorated graph (left). An antenna implementing the concept (right).

been developed in [407. An interesting series of papers on the predesigned gap opening is $221+223$.

The high contrast approach also has its deficiencies. First of all, it is still hard to manipulate the locations and sizes of spectral gaps. Besides, the high contrast approach frequently requires nonphysical values of material parameters.

The moral is that in higher dimensions, it often is hard to create and manipulate the spectral gaps arising due to Bragg scattering (i.e., due to the periodicity of the medium).

A different promising mechanism of opening resonant gaps exists. The author learned about it first from 325. The idea is that spreading infinitely many small identical resonators throughout the medium tends to create spectral gaps around the eigenvalues of the resonator. Regretfully, in the continuous case, this idea apparently has never been made precise. It has been implemented (as the so-called decoration procedure) in its simplest incarnations in the discrete situation [339, as well as in the quantum graph case [27,246]. In practice, more involved spider decoration procedures are desirable, where some internal structure is inserted into each vertex (see discussion in [27, 246]). One can compare this with the more elaborate zig-zag decoration procedure, used to create expander graphs (which boils down to controlling the size of the principal spectral gap [333]).

Recent developments in this direction can be found in [108.

Resonant gaps can also be used to create "slowing down light" optical media (e.g., 192, 251, 361, 400]).

\subsection{Eigenfunction expansion. Let us recall that}

$$
\lambda_{1}(k) \leq \lambda_{2}(k) \leq \lambda_{3}(k) \leq \cdots
$$

is the sequence of eigenvalues of the operator $H(k)$, listed for $k \in \mathbb{R}^{n}$ (with their multiplicity) in a nondecreasing order.

Standard perturbation theory (e.g., 217]) implies that

each $\lambda_{j}(k)$ is a continuous, piecewise analytic function of $k$.

By definition, for each $\lambda_{j}(k)$ the operator $H$ has a generalized (since it is not squaresummable) Bloch eigenfunction $\psi(x)$ with the quasimomentum $k$ (i.e., $\psi(x)=$ $e^{i k \cdot x} p(x)$ with a $\Gamma$-periodic function $\left.p(x)\right)$ :

$$
H \psi=\lambda_{j}(k) \psi .
$$

The question is whether the eigenfunction $\psi$ (and thus the corresponding periodic function $p$ ) can be chosen with a nice dependence of $k$. Whenever the multiplicity 
of the eigenvalue $\lambda_{j}(k)$ stays constant near a point $k_{0}$, one can choose the Bloch eigenfunction analytically depending on $k$. See, e.g., [244,404 for this standard fact. Whenever the band functions collide and thus the multiplicity changes, one cannot extend the Bloch eigenfunction even continuously w.r.t. $k$. However, cutting along these thin bad sets of quasimomenta, one can establish the following:

Lemma 6.7 (Wilcox [394]). There exists a null-set $\mathcal{Z} \subset \mathbb{T}^{*}$ and a sequence $\psi_{j}(k)$ of Bloch eigenfunctions, analytic on $\mathbb{T}^{*} \backslash \mathcal{Z}$, such that $\left\{\psi_{j}(k)\right\}$ form an orthonormal basis in $L^{2}(W)$ for $k \in \mathbb{T}^{*} \backslash \mathcal{Z}$.

This immediately leads to the eigenfunction expansion of any function $f(x) \in$ $L^{2}\left(\mathbb{R}^{n}\right)$ into Bloch eigenfunctions:

Theorem 6.8 ([166], see also [268]). Let $f \in L^{2}\left(\mathbb{R}^{n}\right)$ and

$$
\mathcal{U}_{\Gamma} f(\cdot, k)=\sum_{j} f_{j}(k) \psi_{j}(\cdot, k)
$$

be the expansion of its Floquet transform into the basis $\psi_{j}$. Then

$$
\|f\|_{L^{2}}^{2}=\sum_{j}\left\|f_{j}\right\|_{L^{2}\left(\mathbb{T}^{*}\right)}^{2} .
$$

In other words,

Theorem 6.9. The operator $H$ is unitarily equivalent to the orthogonal direct sum of operators of multiplication by piecewise analytic continuous scalar functions $\lambda_{j}(k)$ in $L^{2}\left(\mathbb{T}^{*}\right)$.

Somewhat weaker results were obtained earlier in 308.

6.3. Absolutely continuous, pure point, and singular continuous parts of the spectrum. According to Theorem 6.9, one needs to understand the spectrum of the operator $A$ acting in $L^{2}\left(\mathbb{T}^{*}\right)$ as multiplication by a continuous piecewise analytic scalar function $a(k)$

$$
(A f)(k)=a(k) f(k) .
$$

Theorem 6.10. The following holds:

(1) The singular continuous spectrum of the operator $A$ is empty:

$$
\sigma_{s c}(A)=\emptyset \text {. }
$$

(2) The pure point spectrum consists of all values $\lambda$ such that the $\lambda$-level set of $a(k)$ has positive measure. In particular, if there is no positive measure flat piece $a=$ const, then $\sigma_{p p}(A)=\emptyset$, and thus the whole spectrum is absolutely continuous:

$$
\sigma(A)=\sigma_{a c}(A) .
$$

(3) For each $\lambda \in \sigma_{p p}(A)$, the function $a(k)$ is constant on an open set.

We thus get the following result:

Corollary 6.11. For any periodic self-adjoint elliptic operator $H$, one has:

(1) The singular continuous spectrum is empty, $\sigma_{s c}(A)=\emptyset$.

(2) The pure point spectrum consists of all values $\lambda$ such that the $\lambda$-level set of some of $\lambda_{j}(k)$ has positive measure. 
(3) If the pure point spectrum is nonempty, then at least one of the band functions $\lambda_{j}$ is constant on an open set.

(4) The pure point spectrum consists of all values $\lambda_{0}$, such that the complex Bloch variety $B_{H, \mathbb{C}}$ contains the flat component $\lambda=\lambda_{0}$.

The last statement of this theorem follows from analyticity of the Bloch variety (Theorem 5.14), which forces any small piece of a constant branch to extend to the whole flat component. Since Theorem 5.19 prohibits such a situation for the Schrödinger operator $-\Delta+V(x)$, one reaches the following conclusion:

Theorem 6.12 (Thomas 382]). Under the conditions of Theorem 5.19, the spectrum of the Schrödinger operator with periodic potential is absolutely continuous.

As was the case with Theorem 5.19 here the condition of boundedness of the periodic potential is significantly stronger than needed (see, e.g., [332, Section VIII.16]). One, however, should not get overexcited about dropping conditions on the coefficients in the highest-order terms, since when they are going below those guaranteeing weak uniqueness of continuation property, one can get point spectrum, as was shown in 144. We will return to this discussion a little bit further in the text.

Since the famous work by L. Thomas [382, all proofs of the absolute continuity of the spectra of periodic elliptic operators followed the same scheme: proving absence of flat components in the dispersion relation (Bloch variety). Due to the usually hard technical work of many researchers, absolute continuity of the spectrum has been proven for many (but still not all) second-order scalar elliptic periodic operators, as well as periodic Pauli, Dirac, elasticity theory, and in some cases Maxwell operators; see [38, 43, 44, 46, 47, 86, 96, 150, 160, 169, 170, 195, 216, 227, 237, 244, 252, 332, 340, 341, 343, 347, 362, 382, 384 and references therein. However, the following conjecture still remains unproven, even under assumption of infinite differentiability of the coefficients of the operator:

Conjecture 6.13. The spectrum of any self-adjoint second-order periodic elliptic scalar operator with nice (e.g., smooth) coefficients is absolutely continuous.

In all works, except [160] and a few papers based upon it (e.g., 384]), the proof goes analogously to that of Theorem 5.19. dominating in the Fourier domain the lower-order terms by the principal part, which gets increasingly hard in the presence of variable coefficients in the first-order terms (magnetic potential), and it does not look feasible at all to treat variable coefficients in the principal part of the operator. The least technical approach, which beautifully avoids these domination estimates and thus allows for variable coefficients in the second-order terms, was due to Friedlander [160. Regretfully, it requires some symmetry condition on the operator, which seems to be superfluous, but no one has succeeded in removing it.

There are also quite a few, often even more demanding, results on absolute continuity for operators in periodic waveguides, on periodic systems of curves and surfaces, etc. 23, 98, 129, 147, 149, 161, 207, 208, 239, 351, 368, 375, 376, 379, .

Remark 6.14. Absolute continuity of the spectrum fails for some periodic elliptic operators of higher order.

Indeed, the Pliś example 329] of a fourth-order elliptic operator which fails unique continuation property, i.e., has a compactly supported eigenfunction, can be easily massaged to produce a periodic example, where $\sigma_{p p} \neq \emptyset[244$. 
This relation to the uniqueness of continuation theorems does not seem accidental. As we have already mentioned, Filonov 144 constructed an example of a second-order periodic elliptic operator with nonempty pure point spectrum, with the leading coefficients falling just below what is needed for the weak uniqueness of continuation to hold. Another evidence of this is the following result:

Theorem 6.15 ([244, Theorems 4.1.5 and 4.1.6]). Existence of an $L^{2}$-eigenfunction for a periodic elliptic operator is equivalent to existence of a (different) superexponentially decaying eigenfunction, i.e., such that

$$
|u(x)| \leq C e^{-|x|^{\gamma}},
$$

with some $\gamma>1$ (depending on the order of the operator and dimension; see details in [244, proof of Theorem 4.1.6]).

Conjecture 6.16. Existence of such a solution must violate some unique continuity at infinity property.

Regretfully, the only such result known that would apply to periodic operators [162,284, is not strong enough to lead to new absolute continuity theorems; e.g., it does not allow variable coefficients in the terms of the first and second orders.

Another confirmation of the relation with the uniqueness of continuation comes from equations on discrete or quantum graphs, where the uniqueness of continuation does not hold. And sure enough, one can find compactly supported eigenfunctions for second-order elliptic periodic operators on such graphs (see [27, and references therein] and Figure [18).

6.3.1. Density of states. An important object in spectral theory and solid state physics is the so-called density of states of a self-adjoint periodic elliptic operator $H$. Here is the idea: consider an "appropriate" sequence of expanding when $N \rightarrow \infty$ domains $V_{N} \subset \mathbb{R}^{n}$, eventually covering the whole space. After imposing appropriate boundary conditions on these domains, one can consider the counting function of eigenvalues $\lambda_{j}$ of the resulting operator with discrete spectrum

$$
\#\left\{\lambda_{j}<\lambda\right\} .
$$

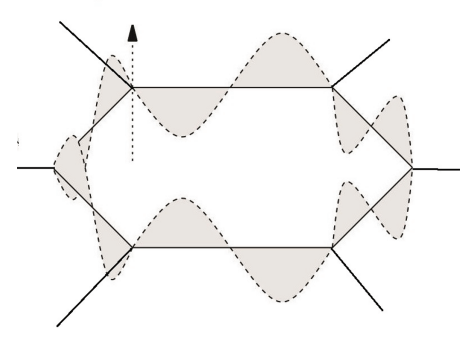

FigURE 18. A compactly supported eigenfunction on a quantum graph. 
Now one normalizes this function by the volume of the domain $V_{N}$ and takes the limit when $N \rightarrow \infty$ :

Definition 6.17. The integrated density of states (IDS) of the operator $H$ is

$$
\rho(\lambda):=\lim _{N \rightarrow \infty} \frac{1}{\left|V_{N}\right|} \#\left\{\lambda_{j}<\lambda\right\}
$$

One certainly wonders whether the limit exists and is independent of the choice of the expanding domains $V_{N}$ and boundary conditions imposed. Under appropriate conditions on those, the answer is "yes" to both questions (see 352, where this is done even for almost periodic coefficients, and [2] for a more general discussion).

Without listing the conditions, we just acknowledge that, assuming that the lattice is transformed to $\mathbb{Z}^{n}$, this is true when one picks $V_{N}$ as the sequence of cubes $\left\{x \in \mathbb{R}^{n}|| x_{j} \mid \leq N, j=1, \ldots, n\right\}$ for $N \in \mathbb{Z}^{+}$and one imposes periodic boundary conditions on those (recall our previous discussion of Born-Karman conditions).

Then a simple calculation shows that the integrated density of states can be described in terms of the dispersion relation $\lambda_{j}(k)$ as follows:

\section{Theorem 6.18.}

$$
\rho(\lambda)=\sum_{j} \mu\left\{k \in \mathbb{T}^{*} \mid \lambda_{j}(k)<\lambda\right\},
$$

where $\mu$ is the previously defined Haar measure on the torus $\mathbb{T}^{*}$.

Due to the analytic nature of the band functions we have studied, one gets:

\section{Corollary 6.19.}

(1) The IDS is a piecewise analytic function of $\lambda$.

(2) Unless a flat band function $\lambda=$ const is present, the IDS is continuous.

(3) Singularities of the IDS can arise if $\lambda$ is a singular or critical value of the dispersion relation (Van Hove singularities).

Definition 6.20. The density of states (DS) is the Radon-Nikodim derivative of the IDS with respect to the Lebesque measure

$$
g(\lambda):=\frac{d \rho}{d \lambda} .
$$

Theorem 6.21.

$$
g(\lambda)=(2 \pi)^{-n} \sum_{j} \int_{\lambda_{j}=\lambda} \frac{d s}{\left|\nabla_{k} \lambda_{j}\right|} .
$$

Noticing that in (6.12) one encounters the integral of a holomorphic form over a real cycle on the Fermi surface $F_{\lambda}$ allows one to use powerful techniques of several complex variables (see [167] and [172,173), which we are unable to address here.

6.4. Spectral asymptotics. Another important and active area of research that we cannot discuss in any detail is high energy spectral asymptotics (e.g., asymptotic of density of states). The reader is referred to the following (incomplete) literature list and references provided there: in 1D [212, 344, 363, 365]; in higher dimensions [52, 159, 194, 214, 235, 264, 265, 294, 297, 298, 318, 320, 357, 364, with the paper [320. being probably the most advanced and up to date. 
6.5. Wannier functions. Bloch functions clearly are analogs for the periodic case of plane waves $e^{i \xi \cdot x}$, which are localized in the Fourier domain. The standard Fourier transform converts the plane waves into delta functions, which are localized in physical space. One wonders whether there is an analog of the delta function basis for the periodic situation, and if yes, whether such functions are useful. The answer is "yes" for both questions, although new nontrivial issues arise here.

The functions in question are called Wannier functions, which are used frequently in numerical computations in solid state physics and photonic crystal theory 61, 62, 279 since they lead to nice discretizations, close to the tight-binding models. One expects (in analogy with the plane waves and delta functions) that they could arise as inverse Floquet transforms w.r.t. the crystal momentum of Bloch functions.

Let us try to be more precise.

Definition 6.22. Suppose that we have a Bloch function $\phi_{k}(x)$ (see (6.2) ) that depends "sufficiently nicely" on the quasimomentum $k$. The corresponding Wannier function is defined as

$$
w(x):=\int_{\mathbb{T}^{*}} \phi_{k}(x) d k .
$$

The standard Fourier series argument shows that smoothness of $\phi_{k}$ with respect to $k$ translates into decay of $w(x)$. For instance, infinite differentiability implies that the $L^{2}$-norm of $w(x)$ on a cube decays faster than algebraically with the shift of the cube to infinity. Analogously, analyticity of $\phi_{k}$ would produce exponential decay of the Wannier function.

Suppose now that one has a multiple band $S$ consisting of $m$ bands of the spectrum, which is separated from the rest of the spectrum by gaps. As discussed in Section 5.12 one obtains an $m$-dimensional analytic Bloch bundle over $\mathbb{T}^{*}$. If this bundle is analytically trivial, it has an analytic basis of Bloch functions: $\phi_{k, 1}, \ldots, \phi_{k, m}$. It is a simple exercise to see (e.g., [249]) that the corresponding exponentially decaying Wannier functions $w_{j}=\int_{\mathbb{T}^{*}} \phi_{k, j} d k$ and all their $\Gamma$-shifts form a basis (which can also be made orthonormal under the appropriate choice of Bloch functions) in the spectral subspace of the operator $H$ that corresponds to the isolated part of the spectrum $S$.

This construction does not work, if the Bloch bundle is not analytically trivial. An incarnation of Oka's principle, due to Grauert [179], shows that the only obstacle is topological: if the Bloch bundle is topologically trivial, then it is automatically analytically trivial (see also 404 for the related discussions). For a while this issue had not been understood and there was a belief that nice Wannier bases always exist, until Thouless showed [383] that in the presence of magnetic terms in a periodic Schrödinger operator, the Bloch bundle can be nontrivial (such nontriviality also arises in topological insulators 28]). Many efforts have been concentrated on establishing sufficient conditions of the triviality, as well as on efficiently finding the Wannier bases (see, e.g., 249, 279, 280, 302, 313, and references therein; 314]).

In the presence of the topological obstacle, however, no basis of (even slowly decaying) Wannier functions exists [249. However, it was shown that instead of the (nonexisting) analytic basis of the bundle, one can always find a nice Parseval frame (i.e., a nice overdetermined system) of exponentially decaying Wannier functions [249. Rather crude estimates on the number of extra functions needed is also provided in [249]. However, in physical dimensions, instead of $m$ families of Wannier functions, one only needs $(m+1)$ of these 12 . 


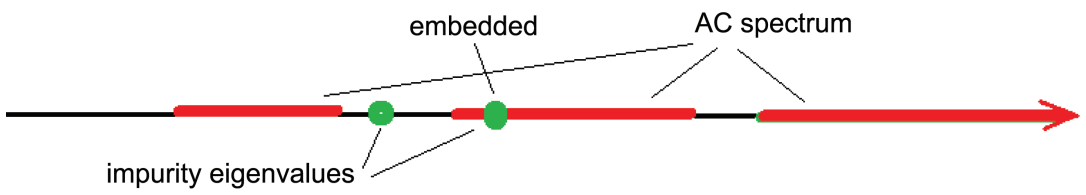

FiguRE 19. Impurity eigenvalues on a band-gap structured spectrum.

6.6. Impurity spectra. Let us return now to the periodic Schrödinger operator $H=-\Delta+V(x)$. An important and frequently studied issue is the existence and properties of the impurity spectrum arising when a localized (compactly supported or sufficiently fast decaying) perturbation $W(x)$ is added to the potential. The well known theorem claims that only eigenvalues of finite multiplicity can appear, leaving the otherwise absolutely continuous spectrum unchanged (see, e.g. [174, Section 18 and references therein]).

Since $V$ is periodic and thus has band-gap structure of the spectrum, these eigenvalues have two options: to appear in the spectral gaps or to embed into the AC spectrum (embedded eigenvalues); see Figure 19.

The general wisdom is that when the perturbation decays sufficiently fast, embedded eigenvalues cannot arise, while if the decay is not fast enough, one can indeed have them. In the case when the background potential $V$ is equal to zero, starting with the famous work by von Neumann and Wigner 390, many results of this nature have been obtained; see, e.g., a nice survey in [118].

If, however, the background periodic potential is present, the issue is far from being resolved. The results confirming the "general wisdom" were obtained in the ODE case 334 337.

Although similar answers are expected in the higher dimensions, proving them happens to be difficult. Some results were obtained in [257 259]. E.g.,

Theorem 6.23 ([257, 258]). Let $n \leq 4$ and let the perturbation $W(x)$ decay exponentially. If for a given $\lambda \in \sigma(H)$ the Fermi surface at that level is irreducible (modulo periodicity), then $\lambda \notin \sigma_{p p}(H+W)$. In particular, if all Fermi surfaces are irreducible, no embedded eigenvalues can arise.

Remark 6.24. Notice that irreducibility of the Fermi surface at a level does not depend on the impurity potential $W$.

As our previous discussion has indicated, it is hard to establish irreducibility of the Fermi surface, although for a second-order periodic ODE it is automatic. One wonders whether the irreducibility condition of this theorem is just an artifact of the techniques used in the proof. It is possible, but does not seem to be very likely. The proof indicated that reducibility could in principle lead to a situation similar to the waveguide theory, where impurity eigenvalues can get embedded. And indeed, there are examples of a periodic fourth-order ODE [315] and of second-order multiperiodic quantum graph operators [345, where reducible Fermi surfaces arise and impurity eigenvalues can be embedded.

In the graph case, due to nontrivial topology, embedded eigenvalues can and do arise. However, 
Theorem 6.25 ([259]). In the graph case, embedded eigenvalues might arise, but if the relevant Fermi surface is irreducible, the corresponding eigenfunction must be supported close to the support of the perturbation; (see details in [259]).

It is interesting to note that in the reducible case, eigenfunctions can spread and indeed have unbounded support 345 .

\section{THRESHOLD EFFECTS}

The name threshold effects (as coined by Birman and Suslina) is used here for the features that depend upon the infinitesimal structure, or maybe just on a finite jet 17 of the dispersion at a spectral edge. An example popular nowadays is of graphene, where the Dirac cones lead to conductance as if governed by the massless Dirac equation [135, 136, 218. We will not dwell on this particular example, but rather list a number of other important relations.

Regretfully, in order to keep the (already excessive) length of the text in check, the author can afford only brief pointers rather than any detailed discussion or formulation of the results.

7.1. Homogenization. Homogenization is an effective medium theory for (long) waves in a highly oscillating periodic (or even random) medium. It is probably the best known threshold effect. Indeed, finding the homogenized (effective medium) operator is, roughly, equivalent to determining the second-order jet of the dispersion relation at the bottom of the spectrum (see, e.g., [5, 22, 39, 40, 74, 75, 77, 78, 205, and references therein). It is sometimes a highly nontrivial procedure (e.g., for the Maxwell operator).

While the usual homogenization leads to a homogeneous medium (which gave the name to the area), and so cannot address finite spectral gaps, more sophisticated approaches (see, e.g., [159, 196, 407]) can achieve this and more.

It is natural to ask whether there is a version of homogenization that occurs near the spectral edges of the internal gaps, where the parabolic shape of the dispersion relation might resemble the one at the bottom of the spectrum. This indeed happens to be the case, as was shown in [37,41]; see also [377, 378.

7.2. Liouville theorems. The classical Liouville theorem says:

Theorem 7.1. Any harmonic function in $\mathbb{R}^{n}$ of polynomial growth of order $N$ is a polynomial of degree $N$. The dimension of this space is

$$
\left(\begin{array}{c}
n+N \\
N
\end{array}\right)-\left(\begin{array}{c}
n+N-2 \\
N-2
\end{array}\right) .
$$

S. T. Yau posed a problem [401] of generalizing this theorem to harmonic functions on noncompact manifolds of nonnegative curvature, which was resolved in 70] (there were also partial contributions from various researchers; see the references in 266, 267]). Thus, finite dimensionality of the spaces of harmonic functions of a given polynomial growth, as well as estimates (rather than exact formulas) for their dimensions, were obtained.

On the other hand, a wonderful observation was made in [15, 299]: the spaces of polynomially growing solutions of periodic divergence type second-order elliptic equations in $\mathbb{R}^{n}$ are finite dimensional. Moreover, their dimensions are given by

\footnotetext{
${ }^{17}$ I.e., truncated Taylor polynomial; see https://en.wikipedia.org/wiki/Jet_(mathematics).
} 
(7.1). Homogenization techniques were used in both cases, which restricted the consideration to the bottom of the spectrum only. In [253, 254, a wide-range generalization of this result was obtained: for an elliptic periodic operator on an abelian covering of a compact manifold (or graph) it was shown that validity of the Liouville theorem at some energy level $\lambda$ is equivalent to the corresponding Fermi surface consisting of finitely many points (i.e., essentially $\lambda$ being at a spectral edge). Moreover, dimensions of the spaces of polynomially growing solutions were explicitly computed in terms of the lowest order of a nontrivial Taylor expansion term of the dispersion relation at the corresponding spectral edge.

7.3. Green's function. Another threshold effect is the behavior of Green's function (the Schwartz kernel of the resolvent) near and at spectral edges. There are well known general resolvent exponential decay estimates with the exponential rate depending upon the distance to the spectrum, e.g., the Combes-Thomas estimates 19,73. However, being applied to self-adjoint periodic elliptic operators, these estimates are not very precise. Indeed, first of all, one expects the exponential decay to be direction dependent, while the operator estimates mentioned above would provide only an isotropic estimate. Secondly, the off-the-spectrum exponential decay must be accompanied by an additional power decaying factor, which the abstract theorems do not provide. On the other hand, at the bottom of the spectrum the dispersion relation of periodic nonmagnetic Schrödinger operator has, as has been mentioned before, a nondegenerate (parabolic) extremum [224, i.e., it resembles the one for the Laplace operator. Thus, one can hope that at least near the spectrum the decay should resemble the one for the Laplacian case, i.e., involving an additional algebraically decaying factor. Moreover, at the edge of the spectrum one expects (in dimensions three and higher) some algebraic decay. And indeed, principal terms were found for the asymptotics of Green's functions of such operators below the spectrum [17, 18, 300] (see also a simplified derivation for the discrete case at the end of the book 395]). These results confirm the expectation.

One can ask the same question near and at the edges of the internal gaps of the spectrum, as long as the dispersion relation has a nondegenerate (parabolic) extremum there. Results of this type were obtained in the recent works [219, 220, 256.

Going inside the spectral bands, it is also natural to mention here the results on the limiting absorption principle for periodic elliptic operators, see, e.g., 42, 167-169,301.

7.4. Impurity spectra in gaps. The local structure of the dispersion relation at a spectral gap edge is also important for understanding the appearance and counting of the impurity eigenvalues that arise due to a localized perturbation of the potential. See 33,36 and references therein.

\section{Solutions}

In this section we provide a very brief overview with pointers to the literature of various results concerning solutions of homogeneous and inhomogeneous periodic elliptic equations.

8.1. Floquet-Bloch expansions. Euler's theorem 128, mentioned in Section 1.1. claims that all solutions of homogeneous constant coefficient linear ODEs (or systems of those) are linear combinations of exponential-polynomial solutions. This 
classical theorem has an extremely nontrivial generalization to the case of constant coefficient systems of PDEs (sometimes called Ehrenpreis's Fundamental Principle) due to works by Ehrenpreis, Malgrange, and Palamodov; see the books 120, 310, 311 devoted to its proof and applications. Here instead of finite linear combinations of exponential-polynomial solutions, one needs to involve the integrals over the characteristic variety of the operator, which in turn relies upon commutative algebra, algebraic geometry, and several complex variable techniques. One can ask the natural question whether there is an analog of this result for periodic elliptic PDEs, providing expansions into Bloch-Floquet solutions. If yes, one expects it to be harder to prove, due to the nonalgebraic nature of the situation (i.e., of the Fermi surface, which replaces the characteristic variety). Nevertheless, somewhat more restrictive than in the constant coefficient case, such results have been obtained in 240,244,247, 260, 312]. Most of the book 244] is devoted to their proofs. Regretfully, probably due to a more restricted nature of these results, they do not seem to have much of consequence. Thus, we do not address them in any detail here.

8.2. Generalized eigenfunctions and Shnol'-Bloch theorems. One of the consequences of the Floquet theory is that for periodic elliptic operators $H$ detecting whether $\lambda$ is in the spectrum is equivalent to existence of a nontrivial Bloch solution $u$ with a real quasimomentum $k$ of the equation $H u=\lambda u$. Since the solution is not square integrable, we would call it a generalized eigenfunction. Since one can easily come up with a generalized eigenfunction (just an exponent) of a Laplace operator for arbitrary $\lambda \in \mathbb{C}$, it is clear that existence of a growing generalized eigenfunction does not necessarily mean being on the spectrum. However, since Bloch solutions with real quasimomenta are bounded, one can ask whether presence of a bounded generalized eigenfunction detects the spectrum. This is indeed the case and the following Bloch theorem (probably never proven by Bloch) holds:

Theorem 8.1 ([244, Section 4.3]). Existence of a nontrivial bounded solution of a periodic elliptic equation $H u=\lambda u$ implies existence of a Bloch solution with a real quasimomentum, and thus $\lambda \in \sigma(H)$.

Indeed, a stronger type of result is known - the so called Shnol' Theorem 83, 174, 244, 346, 353 — which holds also in nonperiodic case. Its simplest version is:

Theorem 8.2. If for any $\epsilon>0$ there exists a nontrivial generalized eigenfunction $u_{\epsilon}$ with the estimate

$$
\left|u_{\epsilon}(x)\right| \leq e^{\epsilon|x|},
$$

then $\lambda \in \sigma(H)$.

Moreover, the following stronger version holds:

Theorem 8.3. If for some $a>0$ there exists a nontrivial generalized eigenfunction $u$ with the estimate

$$
|u(x)| \leq e^{a|x|},
$$

then $\operatorname{dist}(\lambda, \sigma(H)) \leq C \sqrt{e^{2 a}-1}$, where $C$ depends only on the operator $H$. 
A detailed (although somewhat outdated) discussion of the Shnol' theorem can be found in [174, Section 54].

In the periodic elliptic case one can formulate a stronger version 244, Theorem 4.3.1]:

Theorem 8.4. Let $L(x, D)$ be a scalar periodic elliptic operator with smooth coefficients in $\mathbb{R}^{n}$. If $L u=0$ has a nonzero solution satisfying the inequality

$$
|f(x)| \leq C e^{a \sum\left|x_{j}\right|}
$$

for some $a>o$, then it also has a Bloch solution with a quasimomentum $k$ such that $\Im k_{j} \leq$ a for all $j=l, \ldots, n$, that is, a Bloch solution that also satisfies (8.3).

In other words, there is a complex quasimomentum vector, whose distance to the unit torus (and thus dist $(\lambda, \sigma(H))$ ) can be estimated from above.

Remark 8.5. The Shnol' theorem stated with pointwise estimates like in (8.3) fails in non-Euclidean situation; e.g., the hyperbolic Laplacian has a bounded generalized eigenfunction for $\lambda=0$, although 0 is not in the spectrum. An analogous situation occurs for operator on trees. However, analysis of the proof (e.g., in [174]) shows that in fact only an integrated $\left(L^{2}\right)$ estimate of growth is used, which implicitly incorporates the rate of the ball volume growth. Thus, there is an $L^{2}$-version that holds even when the volume of the ball grows exponentially (see [27, Section 3.2] for the graph case).

For further discussions of generalized eigenfunction expansions, the reader can refer to [24,25, 226] and references therein.

8.3. Positive solutions. It was shown in 3 that positive solutions of a periodic elliptic second-order equation in $\mathbb{R}^{n}$ (or on a cocompact abelian covering) allow integral expansions into positive Bloch solutions. See also [244, Section 4.6] for the description of this result, as well as 269] for the case of nilpotent cocompact covering, and 327,328, for related discussions.

8.4. Inhomogeneous equations. It is well known in the theory of periodic ODEs (e.g., [10, 399]) that unique solvability of inhomogeneous equations in $L^{2}$ or in the space of bounded functions is equivalent to the absence of Floquet multipliers of absolute value one (equivalently, absence of Bloch solutions with real quasimomenta). A simple PDE analog of this result (as well as solvability in the spaces of exponentially decaying functions) also holds [244, Section 4.2].

\section{Miscellany}

9.1. Parabolic time-periodic equations. Fluid dynamics problems of stability of periodic flows [402,403. lead naturally to the task of developing an analog of Floquet theory (e.g., completeness of and expansion into Floquet solutions) for parabolic time periodic problems in Banach and Hilbert spaces:

$$
\frac{d x}{d t}=A(t) x, x(0)=x_{0}, A(t+1)=A(t) .
$$

The simplest, albeit already important and nontrivial, example is the heat equation

$$
\frac{d u}{d t}=\Delta_{x} u+b(x, t) u
$$


with time periodic function $b(x, t)$ in an infinite cylinder, with Dirichlet or Neumann boundary conditions. Embarrassingly, there is no general result guaranteeing existence of at least one Floquet solution of (9.2), much less completeness of such solutions. Forget the Lyaponov theorem!

Only in one spatial dimension in an impressive result of this kind for (9.2) achieved in beautiful works [64,65, where the inverse scattering method is used. Nothing comparable is available in higher dimensions, unless severe restrictions are imposed on the periodic term [244, Ch. 5 and references therein]. What seems to be the problem? After all, these equations are hypoelliptic, and the general Floquet theory techniques to a large extent applies to such equations [244, Ch. $3]$ ! The thing is that the general operator theory approach to parabolic periodic equations does not work nearly as nicely as it does for the elliptic case. Namely, as it was discovered by Miloslavskií (see [285, 292] as well as [244, Ch. 5] for the results and discussion), there are extremely nice abstract periodic equations (9.1) with constant highest-order terms, which have no Floquet solutions at all. The positive results known ([285, 292 and [244, Ch. 5 and references therein]) require very strong restrictions on periodic terms, such as $b(x, t)$ in (9.2). The author hopes that the "pathological" nonexistence of Floquet solutions does not hold for (9.2) (all known counterexamples are abstract parabolic equations). A similar difficulty is also encountered in the Floquet theory for evolution equations (9.1) with a bounded operator coefficient $A(t)$ (see [85]).

Some results on positive solutions of periodic parabolic equations have been obtained (see, e.g., [171, 198, 326]).

Time periodic hyperbolic and Schrödinger type equations have also been attracting much attention in physics (e.g., [66, 165, 406]). However, most of the analytic theory described above fails here and other techniques are required and are being developed. See, e.g. the survey 398 and references therein, as well as, e.g., 201, 204, 380, 381, 396, 397.

9.2. Semi-crystals. So far, we have considered infinite periodic structures. The case of the semi-crystal, where a periodic medium occupies a half-space with another (homogeneous or periodic) medium taking the rest of the space, is extremely important. Here one is interested in scattering on and transmission through the semi-crystal, existence of surface (edge) states, etc. One is referred, for instance, to Ch. 5 in the book 213. and references therein (e.g., to Chuburin's work). See also somewhat related works $97,134,155,156,361$. It seems to the author that this topic has not been sufficiently studied yet.

9.3. Photonic crystals. Photonic crystals are artificial periodic optical media that are optical analogs of semi-conductors, which bring about major technological advances (see [111,206, 245] for a nice physics introduction and bibliography, as well as [110,245] for mathematics surveys). The main equation to study here is the Maxwell operator with periodic coefficients. Floquet theory for this operator works in many regards in parallel with the elliptic equations considered in this article, with some notable analytic and numerical quirks (see, e.g., [141, 143, 245, 295] for details and references).

9.4. Waveguides. Periodic waveguides form one of the important applications of Floquet theory. Here one considers boundary value problems in periodically shaped 
domains, where the boundary conditions and the governing equations are also periodic. In some applications, such as quantum waveguides or photonic crystal waveguides, one has to deal also with problems on zero-width surface or curve systems, or where the waves, rather than being confined to the interior of a waveguide, leak (being evanescent) into the surrounding space. Many spectral problems (e.g., absolute continuity of the spectrum or Bethe-Sommerfeld conjecture) happen to be significantly harder in waveguide situations and are not completely understood, in spite of significant progress achieved [57, 98, 100, 161, 200, 244, 348, 351, 368, 375, 376, 379, .

9.5. Coverings and noncommutative versions. As we have mentioned before, one can consider periodic elliptic operators on a normal cocompact Riemannian 18 covering $X \mapsto M$ with a deck group $\Gamma$. If $\Gamma$ is a finitely generated virtually abelian group, the techniques and results of Floquet-Bloch theory described in this text mostly apply (e.g., $3,58,253,254,372$ ), with some caveats such as possible appearance of point spectrum.

Getting rid of the commutativity condition has proven to be hard, due to the difficulties of harmonic analysis on $\Gamma$. And indeed, many techniques fail (after all, the Floquet transform belongs to commutative harmonic analysis) and results do change. There is probably no overarching analog of Floquet theory for all cocompact non-abelian coverings.

Some results do translate to the nilpotent case [269, albeit apparently much more should be possible (e.g., some Liouville type theorems).

In the presence of a periodic magnetic field (but not necessarily periodic magnetic potential), the Schrödinger operator is not periodic anymore, but it commutes with the so-called Zak transformations (magnetic translations), which combine spatial shifts with phase shifts $[13,14,264,305,405]$. Here one deals with the action of the (nilpotent) discrete Heisenberg group $\Gamma$. This case has been intensively studied, due to its physics importance (e.g., [101; 305, and references therein; 357]), but we cannot cover it here.

In more general situations, many issues of spectral theory of periodic Laplacians (and more general operators) on cocompact coverings have been studied, such as relations to the amenability of $\Gamma$ [55], existence of a band-gap structure 60, 373, density of states 2, 119, Shnol'-Bloch theorems 230, presence of pure point spectrum [230], gaps creation [181, 182, 232, 233, 270, 281], absence of a singular continuous spectrum [184, representation of solutions [238, 241,242, etc. See also [55, 59, 183, 185, 371 for related considerations. The reader is reminded that the references in this section are especially far from being comprehensive.

\section{ACKNOWLEDGMENTS}

This work was partially supported by the NSF grant DMS-1517938. The author expresses his deep gratitude to the NSF for its support. The author also would like to thank the Isaac Newton Institute for Mathematical Sciences, Cambridge, for support and hospitality during the program "Periodic and Ergodic Problems", where work on this paper was undertaken. Thanks also go to Professor Susan Friedlander, without whose encouragement this survey most probably would not have seen the light of the day.

\footnotetext{
${ }^{18}$ Or analytic and even discrete.
} 
The author is indebted to many colleagues, co-authors, and former and current students for their publications, discussions, and encouragement, which have made my work on this subject possible over the years. The list of these people is so long, that the author has decided, with apologies to many, to postpone it until the planned monograph. Here I express gratitude to the referee, as well as to Ngoc Do, N. Falkner, N. Filonov, L. Friedlander, T. Kappeler, Yu. Karpeshina, Minh Kha, H. Knörrer, K. Krupchyk, P. Milman, B. Mityagin, Y. Pinchover, L. Parnovskii, B. Simon, R. Shterenberg, A. Sobolev, and T. Suslina for comments, corrections, suggestions, and improvements of pictures. The author is solely responsible for any remaining errors, incompleteness, misnaming the results, etc.

\section{About THE AUTHOR}

Peter Kuchment is professor of mathematics at Texas A\&M University. He is interested in PDEs, mathematical physics, integral geometry, and medical imaging.

\section{ReFERENCES}

[1] M. J. Ablowitz and P. A. Clarkson, Nonlinear evolution equations and inverse scattering, Cambridge University Press, Cambridge, UK, 2001.

[2] T. Adachi and T. Sunada, Density of states in spectral geometry, Comment. Math. Helv. 68 (1993), no. 3, 480-493, DOI 10.1007/BF02565831. MR1236765 (94k:58149)

[3] S. Agmon, On positive solutions of elliptic equations with periodic coefficients in $\mathbf{R}^{n}$, spectral results and extensions to elliptic operators on Riemannian manifolds, Differential equations (Birmingham, Ala. 1983), North-Holland Math. Stud. vol. 92, North-Holland, Amsterdam, 1984, pp. 7-17, DOI 10.1016/S0304-0208(08)73672-7. MR799327 (87a:35060)

[4] S. Agmon, Lectures on elliptic boundary value problems, AMS Chelsea Publishing, Providence, RI, 2010. Prepared for publication by B. Frank Jones, Jr. with the assistance of George W. Batten, Jr.; Revised edition of the 1965 original. MR2589244 (2011c:35004)

[5] G. Allaire and C. Conca, Bloch wave homogenization and spectral asymptotic analysis (English, with English and French summaries), J. Math. Pures Appl. (9) 77 (1998), no. 2, 153-208, DOI 10.1016/S0021-7824(98)80068-8. MR1614641(99d:35017)

[6] G. Allaire, C. Conca, and M. Vanninathan, The Bloch transform and applications, Actes du 29ème Congrès d'Analyse Numérique: CANum'97 (Larnas, 1997), ESAIM Proc. vol. 3, Soc. Math. Appl. Indust., Paris, 1998, pp. 65-84 (electronic), DOI 10.1051/proc:1998040. MR 1642454 (2000a:35006)

[7] V. I. Arnol'd, Ordinary differential equations, MIT Press, Cambridge, Mass.-London, 1978. Translated from the Russian and edited by Richard A. Silverman. MR0508209 (58 \#22707)

[8] V. I. Arnol'd, Geometrical methods in the theory of ordinary differential equations, 2nd ed. Grundlehren der Mathematischen Wissenschaften [Fundamental Principles of Mathematical Sciences], vol. 250, Springer-Verlag, New York, 1988. Translated from the Russian by Joseph Szücs [József M. Szucs]. MR947141(89h:58049)]

[9] V. I. Arnol'd, On the teaching of mathematics (Russian), Uspekhi Mat. Nauk 53 (1998), no. 1(319), 229-234, DOI 10.1070/rm1998v053n01ABEH000005; English transl., Russian Math. Surveys 53 (1998), no. 1, 229-236. MR1618209 (99k:00011)

[10] F. M. Arscott, Periodic differential equations. An introduction to Mathieu, Lamé, and allied functions, International Series of Monographs in Pure and Applied Mathematics, Vol. 66. A Pergamon Press Book, The Macmillan Co., New York, 1964. MR0173798 (30 \#4006)

[11] N. W. Ashcroft and N. D. Mermin, Solid state physics, Holt, Rinehart and Winston, New York-London, 1976.

[12] D. Auckly, J. Corbin, and P. Kuchment, On parseval frames of Wannier functions, in preparation (2015).

[13] L. Auslander, I. Gertner, and R. Tolimieri, The finite Zak transform and the finite Fourier transform, Radar and sonar, Part II, IMA Vol. Math. Appl. vol. 39, Springer, New York, 1992, pp. 21-35, DOI 10.1007/978-1-4684-7832-7_3. MR.1223150 (95b:65162) 
[14] L. Auslander and R. Tolimieri, Abelian harmonic analysis, theta functions and function algebras on a nilmanifold, Lecture Notes in Mathematics, Vol. 436, Springer-Verlag, BerlinNew York, 1975. MR0414785 (54 \#2877)

[15] M. Avellaneda and F.-H. Lin, Un théorème de Liouville pour des équations elliptiques à coefficients périodiques (French, with English summary), C. R. Acad. Sci. Paris Sér. I Math. 309 (1989), no. 5, 245-250. MR1010728 (90j:35072)

[16] J. E. Avron and B. Simon, Analytic properties of band functions, Ann. Physics 110 (1978), no. 1, 85-101. MR0475384(57 \#14992)

[17] M. Babillot, Théorie du renouvellement pour des chaînes semi-markoviennes transientes (French, with English summary), Ann. Inst. H. Poincaré Probab. Statist. 24 (1988), no. 4, 507-569. MR 978023 (90h:60082)

[18] M. Babillot, Asymptotics of Green functions on a class of solvable Lie groups, Potential Anal. 8 (1998), no. 1, 69-100, DOI 10.1023/A:1017991923947. MR:1608646 (99g:60133)

[19] J. M. Barbaroux, J. M. Combes, and P. D. Hislop, Localization near band edges for random Schrödinger operators, Helv. Phys. Acta 70 (1997), no. 1-2, 16-43. Papers honouring the 60th birthday of Klaus Hepp and of Walter Hunziker, Part II (Zürich, 1995). MR1441595 (98h:82028)

[20] G. Barbatis and L. Parnovski, Bethe-Sommerfeld conjecture for pseudodifferential perturbation, Comm. Partial Differential Equations 34 (2009), no. 4-6, 383-418, DOI 10.1080/03605300902769006. MR2530702(2010d:35253)

[21] D. Bättig, A directional compactification of the complex Fermi surface and isospectrality, Séminaire sur les Équations aux Dérivées Partielles, 1989-1990, École Polytech., Palaiseau, 1990, pp. Exp. No. IV, 11. MR1073179 (91m:58160)

[22] A. Bensoussan, J.-L. Lions, and G. Papanicolaou, Asymptotic analysis for periodic structures, Studies in Mathematics and its Applications, vol. 5, North-Holland Publishing Co., Amsterdam-New York, 1978. MR503330(82h:35001)

[23] F. Bentosela, P. Duclos, and P. Exner, Absolute continuity in periodic thin tubes and strongly coupled leaky wires, Lett. Math. Phys. 65 (2003), no. 1, 75-82, DOI 10.1023/A:1027362115133. MR2019393(2004k:81119)

[24] Ju. M. Berezans'kiǔ, Expansions in eigenfunctions of selfadjoint operators, Translated from the Russian by R. Bolstein, J. M. Danskin, J. Rovnyak and L. Shulman. Translations of Mathematical Monographs, Vol. 17, American Mathematical Society, Providence, R.I. 1968. MR0222718 (36 \#5768)

[25] F. A. Berezin and M. A. Shubin, The Schrödinger equation, Mathematics and its Applications (Soviet Series), vol. 66, Kluwer Academic Publishers Group, Dordrecht, 1991. Translated from the 1983 Russian edition by Yu. Rajabov, D. A. Leĭtes and N. A. Sakharova and revised by Shubin; With contributions by G. L. Litvinov and Leĭtes. MR 1186643(93i:81001)

[26] G. Berkolaiko and A. Comech, Symmetry and Dirac points in graphene spectrum, arXiv: 1412.8096 (2014).

[27] G. Berkolaiko and P. Kuchment, Introduction to quantum graphs, Mathematical Surveys and Monographs, vol. 186, American Mathematical Society, Providence, RI, 2013. MR.3013208

[28] B. A. Bernevig, Topological insulators and topological superconductors, Princeton University Press, Princeton, NJ, 2013. With Taylor L. Hughes. MR 3185492

[29] M. V. Berry and M. Wilkinson, Diabolical points in the spectra of triangles, Proc. Roy. Soc. London Ser. A 392 (1984), no. 1802, 15-43. MR738925 (85e:81021)

[30] H. Bethe and A. Sommerfeld, Elektronentheorie der metalle, Handbuch der Physik (H. Geiger and K. Scheel, eds.), vol. 24,2, Springer, 1933, This nearly 300-page chapter was later published as a separate book: Elektronentheorie der Metalle (Springer, 1967), pp. 333-622.

[31] E. Bierstone and P. D. Milman, Semianalytic and subanalytic sets, Inst. Hautes Études Sci. Publ. Math. 67 (1988), 5-42. MR972342(89k:32011)

[32] E. Bierstone and P. D. Milman, A simple constructive proof of canonical resolution of singularities, Effective methods in algebraic geometry (Castiglioncello, 1990), Progr. Math. vol. 94, Birkhäuser Boston, Boston, MA, 1991, pp. 11-30, DOI 10.1007/978-1-4612-0441-1_2. MR:1106412(92h:32053)

[33] M. Sh. Birman, On a discrete spectrum in gaps of a second-order perturbed periodic operator (Russian), Funktsional. Anal. i Prilozhen. 25 (1991), no. 2, 89-92, DOI 10.1007/BF01079605; English transl., Funct. Anal. Appl. 25 (1991), no. 2, 158-161. MR1142222 (92m:47090) 
[34] M. Sh. Birman, The discrete spectrum in gaps of the perturbed periodic Schrödinger operator. I. Regular perturbations, Boundary value problems, Schrödinger operators, deformation quantization, Math. Top., vol. 8, Akademie Verlag, Berlin, 1995, pp. 334-352. MR1389015 (97d:47055)

[35] M. Sh. Birman, The discrete spectrum of the periodic Schrödinger operator perturbed by a decreasing potential, Algebra i Analiz 8 (1996), no. 1, 3-20. MR1392011 (97h:47047)

[36] M. Sh. Birman, The discrete spectrum in gaps of the perturbed periodic Schrödinger operator. II. Nonregular perturbations, Algebra i Analiz 9 (1997), no. 6, 62-89. MR.1610239 (99h:47054)

[37] M. Sh. Birman, On the homogenization for periodic operators in a neighborhood of an edge of an internal gap, Algebra i Analiz 15 (2003), no. 4, 61-71. MR2068979 (2006i:35010)

[38] M. Sh. Birman and T. A. Suslina, A periodic magnetic Hamiltonian with a variable metric. The problem of absolute continuity (Russian, with Russian summary), Algebra i Analiz 11 (1999), no. 2, 1-40; English transl., St. Petersburg Math. J. 11 (2000), no. 2, 203-232. MR.1702587 (2000i:35026)

[39] M. Sh. Birman and T. A. Suslina, Threshold effects near the lower edge of the spectrum for periodic differential operators of mathematical physics, Systems, approximation, singular integral operators, and related topics (Bordeaux, 2000), Oper. Theory Adv. Appl. vol. 129, Birkhäuser, Basel, 2001, pp. 71-107. MR.1882692 (2003f:35220)

[40] M. Sh. Birman and T. A. Suslina, Periodic second-order differential operators. Threshold properties and averaging (Russian, with Russian summary), Algebra i Analiz 15 (2003), no. 5, 1-108, DOI 10.1090/S1061-0022-04-00827-1; English transl., St. Petersburg Math. J. 15 (2004), no. 5, 639-714. MR2068790 (2005k:47097)

[41] M. Sh. Birman and T. A. Suslina, Homogenization of a multidimensional periodic elliptic operator in a neighborhood of an edge of an inner gap (Russian, with English and Russian summaries), Zap. Nauchn. Sem. S.-Peterburg. Otdel. Mat. Inst. Steklov. (POMI) 318 (2004), no. Kraev. Zadachi Mat. Fiz. i Smezh. Vopr. Teor. Funkts. 36 [35], 60-74, 309, DOI 10.1007/s10958-006-0192-9; English transl., J. Math. Sci. (N. Y.) 136 (2006), no. 2, 3682-3690. MR2120232(2005j:35006)

[42] M. Sh. Birman and T. A. Suslina, The limiting absorption principle and the homogenization procedure for periodic elliptic operators (Russian), Funktsional. Anal. i Prilozhen. 42 (2008), no. 4, 105-108, DOI 10.1007/s10688-008-0047-x; English transl., Funct. Anal. Appl. 42 (2008), no. 4, 336-339. MR 2492431(2010f:35016)

[43] M. Sh. Birman, T. A. Suslina, and R. G. Shterenberg, Absolute continuity of the twodimensional Schrödinger operator with delta potential concentrated on a periodic system of curves (Russian, with Russian summary), Algebra i Analiz 12 (2000), no. 6, 140-177; English transl., St. Petersburg Math. J. 12 (2001), no. 6, 983-1012. MR.1816514 (2002k:35227)

[44] M. Sh. Birman and T. A. Suslina, The periodic Dirac operator is absolutely continuous, Integral Equations Operator Theory 34 (1999), no. 4, 377-395, DOI 10.1007/BF01272881. MR 1702229 (2000h:47068)

[45] M. Sh. Birman and T. A. Suslina, Two-dimensional periodic Pauli operator. The effective masses at the lower edge of the spectrum, Mathematical results in quantum mechanics (Prague, 1998), Oper. Theory Adv. Appl., vol. 108, Birkhäuser, Basel, 1999, pp. 13-31. MR 1708785 (2000g:81049)

[46] M. Sh. Birman and T. A. Suslina, On the absolute continuity of the periodic Schrödinger and Dirac operators with magnetic potential, Differential equations and mathematical physics (Birmingham, AL, 1999), AMS/IP Stud. Adv. Math., vol. 16, Amer. Math. Soc., Providence, RI, 2000, pp. 41-49. MR1764740

[47] M. Sh. Birman and T. A. Suslina, Absolute continuity of the spectrum of the periodic operator of elasticity theory for constant shear modulus, Nonlinear problems in mathematical physics and related topics, II, Int. Math. Ser. (N. Y.) vol. 2, Kluwer/Plenum, New York, 2002, pp. 69-74, DOI 10.1007/978-1-4615-0701-7_4. MR1971990 (2004c:35296)

[48] G. Borg, Eine Umkehrung der Sturm-Liouvilleschen Eigenwertaufgabe. Bestimmung der Differentialgleichung durch die Eigenwerte (German), Acta Math. 78 (1946), 1-96. MR0015185 (7,382d)

[49] D. I. Borisov and K. V. Pankrashkin, On the extrema of band functions in periodic waveguides (Russian), Funktsional. Anal. i Prilozhen. 47 (2013), no. 3, 87-90; English transl., Funct. Anal. Appl. 47 (2013), no. 3, 238-240. MR3154842 
[50] D. Borisov and K. Pankrashkin, Quantum waveguides with small periodic perturbations: gaps and edges of Brillouin zones, J. Phys. A 46 (2013), no. 23, 235203, 18, DOI 10.1088/17518113/46/23/235203. MR3064364

[51] L. P. Bouckaert, R. Smoluchowski, and E. Wigner, Theory of brillouin zones and symmetry properties of wave functions in crystals, Phys. Rev. 50 (1936), 58-67.

[52] O. Bratteli, P. E. T. Jørgensen, and D. W. Robinson, Spectral asymptotics of periodic elliptic operators, Math. Z. 232 (1999), no. 4, 621-650, DOI 10.1007/PL00004773. MR1727545 (2000k:35223)

[53] L. Brillouin, Wave propagation in periodic structures. Electric filters and crystal lattices, Dover Publications, Inc., New York, N. Y. 1953. 2d ed. MR0052978 (14,704a)

[54] L. Brillouin and M. Parodi, Propagation des ondes dans les milieux périodiques (French), Masson et Cie, Paris; Dunod, Paris, 1956. MR0079952 (18,170f)

[55] R. Brooks, The fundamental group and the spectrum of the Laplacian, Comment. Math. Helv. 56 (1981), no. 4, 581-598, DOI 10.1007/BF02566228. MR656213 (84j:58131)

[56] B. M. Brown, M. S. P. Eastham, and K. M. Schmidt, Periodic differential operators, Operator Theory: Advances and Applications, vol. 230, Birkhäuser/Springer Basel AG, Basel, 2013. MR2978285

[57] B. M. Brown, V. Hoang, M. Plum, and I. G. Wood, Floquet-Bloch theory for elliptic problems with discontinuous coefficients, Spectral theory and analysis, Oper. Theory Adv. Appl. vol. 214, Birkhäuser/Springer Basel AG, Basel, 2011, pp. 1-20, DOI 10.1007/978-3-76439994-8_1. MR2808460(2012i:35067)

[58] J. Brüning, P. Exner, and V. A. Geyler, Large gaps in point-coupled periodic systems of manifolds, J. Phys. A 36 (2003), no. 17, 4875-4890, DOI 10.1088/0305-4470/36/17/314. MR $1984016(2005 f: 81080)$

[59] J. Brüning and T. Sunada, On the spectrum of gauge-periodic elliptic operators, Astérisque 210 (1992), 6, 65-74. Méthodes semi-classiques, Vol. 2 (Nantes, 1991). MR 1221352 (94j:58170)

[60] J. Brüning and T. Sunada, On the spectrum of periodic elliptic operators, Nagoya Math. J. 126 (1992), 159-171. MR 1171598(93f:58235)

[61] K. Busch, G. von Freymann, S. Linden, S. F. Mingaleev, L. Tkeshelashvili, and M. Wegener, Periodic nanostructures for photonics, Physics Reports 444 (2007), no. 3-6, 101-202.

[62] K. Busch, S. Mingaleev, A. Garcia-Martin, M. Schillinger, and D. Hermann, The Wannier function approach to photonic crystal circuits, Journal of Physics: Condensed Matter 15 (2003), no. 30, R1233.

[63] J. Callaway, Energy band theory, Pure and Applied Physics, Vol. 16, Academic Press, New York-London, 1964. MR0162578 (28 \#5776)

[64] S.-N. Chow, K. Lu, and J. Mallet-Paret, Floquet theory for parabolic differential equations, J. Differential Equations 109 (1994), no. 1, 147-200, DOI 10.1006/jdeq.1994.1047. MR1272403 (95c:35116)

[65] S.-N. Chow, K. Lu, and J. Mallet-Paret, Floquet bundles for scalar parabolic equations, Arch. Rational Mech. Anal. 129 (1995), no. 3, 245-304, DOI 10.1007/BF00383675. MR.1328478 (96c:35070)

[66] S.-I. Chu, Nonperturbative approaches to atomic and molecular multiphoton (half-collision) processes in intense laser fields, Multiparticle quantum scattering with applications to nuclear, atomic and molecular physics (Minneapolis, MN, 1995), IMA Vol. Math. Appl. vol. 89, Springer, New York, 1997, pp. 343-387, DOI 10.1007/978-1-4612-1870-8_13. MR1487928 (98i:81291)

[67] F. R. K. Chung, Spectral graph theory, CBMS Regional Conference Series in Mathematics, vol. 92, Published for the Conference Board of the Mathematical Sciences, Washington, DC; by the American Mathematical Society, Providence, RI, 1997. MR1421568 (97k:58183)

[68] E. A. Coddington and R. Carlson, Linear ordinary differential equations, Society for Industrial and Applied Mathematics (SIAM), Philadelphia, PA, 1997. MR1450591 (99j:34001)

[69] E. A. Coddington and N. Levinson, Theory of ordinary differential equations, McGraw-Hill Book Company, Inc., New York-Toronto-London, 1955. MR0069338 (16,1022b)

[70] T. H. Colding and W. P. Minicozzi II, Harmonic functions on manifolds, Ann. of Math. (2) 146 (1997), no. 3, 725-747, DOI 10.2307/2952459. MR:1491451 (98m:53052) 
[71] Y. Colin de Verdière, Sur les singularités de van Hove génériques (French), Mém. Soc. Math. France (N.S.) 46 (1991), 99-110. Analyse globale et physique mathématique (Lyon, 1989). MR 1125838 (92h:35160)

[72] Y. Colin de Verdière and Th. Kappeler, On double eigenvalues of Hill's operator, J. Funct. Anal. 86 (1989), no. 1, 127-135, DOI 10.1016/0022-1236(89)90067-0. MR.1013936 (91b:34143)

[73] J. M. Combes and L. Thomas, Asymptotic behaviour of eigenfunctions for multiparticle Schrödinger operators, Comm. Math. Phys. 34 (1973), 251-270. MR0391792(52 \#12611)

[74] C. Conca, R. Orive, and M. Vanninathan, Bloch approximation in homogenization and applications, SIAM J. Math. Anal. 33 (2002), no. 5, 1166-1198 (electronic), DOI 10.1137/S0036141001382200. MR.1897707 (2003d:35015)

[75] C. Conca, R. Orive, and M. Vanninathan, Bloch approximation in homogenization on bounded domains, Asymptot. Anal. 41 (2005), no. 1, 71-91. MR2124894 (2006g:35011)

[76] C. Conca, J. Planchard, and M. Vanninathan, Fluids and periodic structures, RAM: Research in Applied Mathematics, vol. 38, John Wiley \& Sons, Ltd., Chichester; Masson, Paris, 1995. MR 1652238 (99k:73094)

[77] C. Conca and M. Vanninathan, Homogenization of periodic structures via Bloch decomposition, SIAM J. Appl. Math. 57 (1997), no. 6, 1639-1659, DOI 10.1137/S0036139995294743. MR 1484944 (98j:35017)

[78] C. Conca and M. Vanninathan, Fourier approach to homogenization problems, ESAIM Control Optim. Calc. Var. 8 (2002), 489-511, DOI 10.1051/cocv:2002048. A tribute to J. L. Lions. MR1932961 (2003j:35017)

[79] L. J. Corwin and F. P. Greenleaf, Representations of nilpotent Lie groups and their applications. Part I, Cambridge Studies in Advanced Mathematics, vol. 18, Cambridge University Press, Cambridge, 1990. Basic theory and examples. MR 1070979 (92b:22007)

[80] A. P. Cracknell, The Fermi surfaces of metals, Taylor \& Francis, London, 1971.

[81] A. P. Cracknell and K. S. Wong, The Fermi surface, Claredon Press, Oxford, 1973.

[82] R. V. Craster, J. Kaplunov, E. Nolde, and S. Guenneau, Bloch dispersion and high frequency homogenization for separable doubly-periodic structures, Wave Motion 49 (2012), no. 2, 333346, DOI 10.1016/j.wavemoti.2011.11.005. MR.2890754

[83] H. L. Cycon, R. G. Froese, W. Kirsch, and B. Simon, Schrödinger operators with application to quantum mechanics and global geometry, Springer Study Edition, Texts and Monographs in Physics, Springer-Verlag, Berlin, 1987. MR883643 (88g:35003)

[84] B. E. J. Dahlberg and E. Trubowitz, A remark on two-dimensional periodic potentials, Comment. Math. Helv. 57 (1982), no. 1, 130-134, DOI 10.1007/BF02565850. MR672849 (84h:35119)

[85] Ju. L. Dalec'kiı̌ and M. G. Kreĭn, Stability of solutions of differential equations in Banach space, American Mathematical Society, Providence, R.I. 1974. Translated from the Russian by S. Smith; Translations of Mathematical Monographs, Vol. 43. MR0352639(50 \#5126)

[86] L. I. Danilov, On the spectrum of the Dirac operator in $\mathbf{R}^{n}$ with periodic potential (Russian, with English summary), Teoret. Mat. Fiz. 85 (1990), no. 1, 41-53, DOI 10.1007/BF01017245; English transl., Theoret. and Math. Phys. 85 (1990), no. 1, 10391048 (1991). MR 1083951(92a:35119)

[87] L. I. Danilov, Resolvent estimates and the spectrum of the Dirac operator with a periodic potential (Russian, with English and Russian summaries), Teoret. Mat. Fiz. 103 (1995), no. 1, 3-22, DOI 10.1007/BF02069779; English transl., Theoret. and Math. Phys. 103 (1995), no. 1, 349-365. MR1470934 (98f:35112)

[88] L. I. Danilov, On the spectrum of the two-dimensional periodic Dirac operator (Russian, with Russian summary), Teoret. Mat. Fiz. 118 (1999), no. 1, 3-14, DOI 10.1007/BF02557191; English transl., Theoret. and Math. Phys. 118 (1999), no. 1, 1-11. MR.1702856 (2000h:35117)

[89] L. I. Danilov, Absolute continuity of the spectrum of a periodic Dirac operator (Russian, with Russian summary), Differ. Uravn. 36 (2000), no. 2, 233-240, 287, DOI 10.1007/BF02754212; English transl., Differ. Equ. 36 (2000), no. 2, 262-271. MR1773794 (2001f:47082)

[90] L. I. Danilov, On the spectrum of the periodic Dirac operator (Russian, with Russian summary), Teoret. Mat. Fiz. 124 (2000), no. 1, 3-17, DOI 10.1007/BF02551063; English transl., Theoret. and Math. Phys. 124 (2000), no. 1, 859-871. MR1821309 (2002b:81028) 
[91] L. I. Danilov, On the absolute continuity of the spectrum of a periodic Schrödinger operator (Russian, with Russian summary), Mat. Zametki 73 (2003), no. 1, 49-62, DOI 10.1023/A:1022169916738; English transl., Math. Notes 73 (2003), no. 1-2, 46-57. MR.1993539 (2004f:35130)

[92] L. I. Danilov, On the spectrum of the two-dimensional periodic Schrödinger operator (Russian, with Russian summary), Teoret. Mat. Fiz. 134 (2003), no. 3, 447-459, DOI 10.1023/A:1022605623235; English transl., Theoret. and Math. Phys. 134 (2003), no. 3, 392-403. MR2001818(2004j:35210)

[93] L. I. Danilov, On the nonexistence of eigenvalues in the spectrum of a generalized twodimensional periodic Dirac operator (Russian, with Russian summary), Algebra i Analiz 17 (2005), no. 3, 47-80, DOI 10.1090/S1061-0022-06-00911-3; English transl., St. Petersburg Math. J. 17 (2006), no. 3, 409-433. MR2167843 (2006m:35261)

[94] L. I. Danilov, On absolute continuity of the spectrum of a periodic magnetic Schrödinger operator, J. Phys. A 42 (2009), no. 27, 275204, 20, DOI 10.1088/1751-8113/42/27/275204. MR 2512122(2010f:81090)

[95] L. I. Danilov, On absolute continuity of the spectrum of three-and four-dimensional periodic Schrödinger operators, J. Phys. A 43 (2010), no. 21, 215201, 13, DOI 10.1088/17518113/43/21/215201. MR2644606(2011i:81056)

[96] L. I. Danilov, On absolute continuity of the spectrum of a 3D periodic magnetic Dirac operator, Integral Equations Operator Theory 71 (2011), no. 4, 535-556, DOI 10.1007/s00020011-1906-z. MR2854864

[97] E. B. Davies and B. Simon, Scattering theory for systems with different spatial asymptotics on the left and right, Comm. Math. Phys. 63 (1978), no. 3, 277-301. MR513906 (80c:81110)

[98] V. I. Derguzov, A mathematical investigation of periodic cylindrical wave guides. I, II (Russian, with English summary), Vestnik Leningrad. Univ. No. 13, Mat. Meh. Astronom. Vyp. 3 (1972), 32-40, 149; ibid. No. 19 Mat. Meh. Astronom. Vyp. 3 (1972), 14-20, 145. MR0340856 (49 \#5606)

[99] V. I. Derguzov, Linear equations with periodic coefficients and their applications to wave guide systems, Ph.D. thesis, Leningrad, 1975, (In Russian).

[100] V. I. Derguzov, On the discrete spectrum of a periodic boundary value problem connected with the study of periodic waveguides (Russian), Sibirsk. Mat. Zh. 21 (1980), no. 5, 27-38, 189. MR 592214 (82g:78023)

[101] E. I. Dinaburg, Ya. G. Sinai, and A. B. Soshnikov, Splitting of the low Landau levels into a set of positive Lebesgue measure under small periodic perturbations, Comm. Math. Phys. 189 (1997), no. 2, 559-575, DOI 10.1007/s002200050217. MR1480033 (98j:81079)

[102] J. Dixmier, Les algèbres d'opérateurs dans l'espace hilbertien (Algèbres de von Neumann) (French), Cahiers scientifiques, Fascicule XXV, Gauthier-Villars, Paris, 1957. MR 0094722 (20 \#1234)

[103] P. Djakov and B. Mityagin, Smoothness of Schrödinger operator potential in the case of Gevrey type asymptotics of the gaps, J. Funct. Anal. 195 (2002), no. 1, 89-128, DOI 10.1006/jfan.2002.3975. MR.1934354(2003k:34167)

[104] P. Djakov and B. S. Mityagin, Instability zones of one-dimensional periodic Schrödinger and Dirac operators (Russian, with Russian summary), Uspekhi Mat. Nauk 61 (2006), no. 4(370), 77-182, DOI 10.1070/RM2006v061n04ABEH004343; English transl., Russian Math. Surveys 61 (2006), no. 4, 663-766. MR2279044(2007i:47054)

[105] P. Djakov and B. Mityagin, Spectral gaps of Schrödinger operators with periodic singular potentials, Dyn. Partial Differ. Equ. 6 (2009), no. 2, 95-165, DOI 10.4310/DPDE.2009.v6.n2.a1. MR2542499 (2010g:47089)

[106] N. Do, On the quantum graph spectra of graphyne nanotubes, Anal. Math. Phys. 5 (2015), no. 1, 39-65, DOI 10.1007/s13324-014-0069-x. MR3305430

[107] Ngoc T. Do and P. Kuchment, Quantum graph spectra of a graphyne structure, Nanoscale Systems: Mathematical Modeling, Theory and Applications 2 (2013), no. 1, 107-123.

[108] Ngoc T. Do, P. Kuchment, and B. Ong. On resonant spectral gap opening in quantum graph networks arXiv:1601.04774 (2016), to appear.

[109] Ngoc T. Do, P. Kuchment, and F. Sottile, On the generic structure of spectral edges for periodic difference operators, In preparation (2015).

[110] W. Dörfler, A. Lechleiter, M. Plum, G. Schneider, and C. Wieners, Photonic crystals: Mathematical analysis and numerical approximation, Oberwolfach SEminars, vol. 42. 
[111] J. P. Dowling, Photonic and acoustic band-gap bibliography, http://phys.lsu.edu/ jdowling/pbgbib.html.

[112] B. A. Dubrovin, I. M. Krichever, and S. P. Novikov, Integrable systems. I [MR0842910 (87k:58112)], Dynamical systems, IV, Encyclopaedia Math. Sci. vol. 4, Springer, Berlin, 2001, pp. 177-332, DOI 10.1007/978-3-662-06791-8_3. MR1866633

[113] B. A. Dubrovin, I. M. Krichever, and S. P. Novikov, Topological and algebraic geometry methods in contemporary mathematical physics, Classic Reviews in Mathematics and Mathematical Physics, vol. 2, Cambridge Scientific Publishers, Cambridge, 2004. MR2187099 (2006f:37002)

[114] N. Dunford and J. T. Schwartz, Linear operators. Part II, Wiley Classics Library, John Wiley \& Sons, Inc., New York, 1988. Spectral theory. Selfadjoint operators in Hilbert space; With the assistance of William G. Bade and Robert G. Bartle; Reprint of the 1963 original; A Wiley-Interscience Publication. MR 1009163 (90g:47001b)

[115] V. V. Dyakin and S. I. Petrukhnovskiŭ, Some geometric properties of Fermi surfaces (Russian), Dokl. Akad. Nauk SSSR 264 (1982), no. 5, 1117-1119. MR672029(84h:81120)

[116] V. V. Dyakin and S. I. Petrukhnovskiu, On the discreteness of the spectrum of some operator pencils connected with the periodic Schrödinger equation (Russian, with English summary), Teoret. Mat. Fiz. 74 (1988), no. 1, 94-102, DOI 10.1007/BF01018212; English transl., Theoret. and Math. Phys. 74 (1988), no. 1, 66-72. MR 940464 (89k:35163)

[117] M. S. P. Eastham, The spectral theory of periodic differential equations, Texts in Mathematics (Edinburgh), Scottish Academic Press, Edinburgh; Hafner Press, New York, 1973. MR.3075381

[118] M. S. P. Eastham and H. Kalf, Schrödinger-type operators with continuous spectra, Research Notes in Mathematics, vol. 65, Pitman (Advanced Publishing Program), Boston, Mass.London, 1982. MR667015 (84i:35107)

[119] D. V. Efremov and M. A. Shubin, The spectral asymptotics of elliptic operators of Schrödinger type on a hyperbolic space, J. Soviet Math. 60 (1992), no. 5, 1637-1662, DOI 10.1007/BF01097529. MR1181097 (94d:58145)

[120] L. Ehrenpreis, Fourier analysis in several complex variables, Pure and Applied Mathematics, Vol. XVII, Wiley-Interscience Publishers A Division of John Wiley \& Sons, New YorkLondon-Sydney, 1970. MR0285849 (44 \#3066)

[121] D. M. Elton, The Bethe-Sommerfield conjecture for the 3-dimensional periodic Landau operator, Rev. Math. Phys. 16 (2004), no. 10, 1259-1290, DOI 10.1142/S0129055X04002242. MR $2114599(2005 \mathrm{k}: 81070)$

[122] A. Enyanshin and A. Ivanovskii, Graphene allotropes: stability, structural and electronic properties from df-tb calculations, Phys. Status Solidi (B) 248 (2011), 1879-1883.

[123] G. Eskin, Inverse spectral problem for the Schrödinger equation with periodic vector potential, Comm. Math. Phys. 125 (1989), no. 2, 263-300. MR1016872 (91a:35052)

[124] G. Eskin, Inverse spectral problem for the Schrödinger operator with periodic magnetic and electric potentials, Séminaire sur les Équations aux Dérivées Partielles, 1988-1989, École Polytech., Palaiseau, 1989, pp. Exp. No. XIII, 6. MR 1032289 (91a:58193)

[125] G. Eskin, J. Ralston, and E. Trubowitz, Isospectral periodic potentials on $\mathbf{R}^{n}$, Inverse problems (New York, 1983), SIAM-AMS Proc. vol. 14, Amer. Math. Soc., Providence, RI, 1984, pp. 91-96. MR773705

[126] G. Eskin, J. Ralston, and E. Trubowitz, The multidimensional inverse spectral problem with a periodic potential, Microlocal analysis (Boulder, Colo. 1983), Contemp. Math. vol. 27, Amer. Math. Soc., Providence, RI, 1984, pp. 45-56, DOI 10.1090/conm/027/741038. MR:741038(85k:58079)

[127] G. Eskin, J. Ralston, and E. Trubowitz, On isospectral periodic potentials in $\mathbf{R}^{n}$. II, Comm. Pure Appl. Math. 37 (1984), no. 6, 715-753, DOI 10.1002/cpa.3160370602. MR762871 (86e:35109b)

[128] L. Euler, De integratione aequationum differentialium altiorum gradum, Miscellanea berol. 7 (1743), 193-242.

[129] P. Exner and R. L. Frank, Absolute continuity of the spectrum for periodically modulated leaky wires in $\mathbb{R}^{3}$, Ann. Henri Poincaré 8 (2007), no. 2, 241-263, DOI 10.1007/s00023-0060307-3. MR 2314447 (2008a:81063) 
[130] P. Exner, P. Kuchment, and B. Winn, On the location of spectral edges in $\mathbb{Z}$-periodic media, J. Phys. A 43 (2010), no. 47, 474022, 8, DOI 10.1088/1751-8113/43/47/474022. MR 2738117 (2011m:81113)

[131] H. Federer, Geometric measure theory, Die Grundlehren der mathematischen Wissenschaften, Band 153, Springer-Verlag New York Inc., New York, 1969. MR0257325 (41 \#1976)

[132] C. L. Fefferman, J. P. Lee-Thorp, and M. I. Weinstein, Topologically protected states in onedimensional continuous systems and Dirac points, Proc. Natl. Acad. Sci. USA 111 (2014), no. 24, 8759-8763, DOI 10.1073/pnas.1407391111. MR3263410

[133] C. L. Fefferman, J. P. Lee-Thorp, and M. I. Weinstein, Bifurcations of edge states - topologically protected and non-protected - in continuous 2d honeycomb structures, arXiv:1509. 08957 (2015).

[134] C. L. Fefferman, J. P. Lee-Thorp, and M. I. Weinstein, Edge states in honeycomb structures, arXiv: $1506.06111(2015)$.

[135] C. L. Fefferman and M. I. Weinstein, Honeycomb lattice potentials and Dirac points, J. Amer. Math. Soc. 25 (2012), no. 4, 1169-1220, DOI 10.1090/S0894-0347-2012-00745-0. MR2947949

[136] C. L. Fefferman and M. I. Weinstein, Wave packets in honeycomb structures and twodimensional Dirac equations, Comm. Math. Phys. 326 (2014), no. 1, 251-286, DOI 10.1007/s00220-013-1847-2. MR3162492

[137] J. Feldman, H. Knörrer, and E. Trubowitz, The perturbatively stable spectrum of a periodic Schrödinger operator, Invent. Math. 100 (1990), no. 2, 259-300, DOI 10.1007/BF01231187. MR 1047135 (91m:35167)

[138] J. Feldman, H. Knörrer, and E. Trubowitz, Perturbatively unstable eigenvalues of a periodic Schrödinger operator, Comment. Math. Helv. 66 (1991), no. 4, 557-579, DOI 10.1007/BF02566665. MR 1129797 (92k:35067)

[139] J. Feldman, H. Knörrer, and E. Trubowitz, There is no two-dimensional analogue of Lamé's equation, Math. Ann. 294 (1992), no. 2, 295-324, DOI 10.1007/BF01934328. MR.1183408 (93m:35133)

[140] The Fermi Surface Database, http://www.phys.ufl.edu/fermisurface/.

[141] A. Figotin and P. Kuchment, Band-gap structure of spectra of periodic dielectric and acoustic media. I. Scalar model, SIAM J. Appl. Math. 56 (1996), no. 1, 68-88, DOI 10.1137/S0036139994263859. MR1372891 (97g:35122)

[142] A. Figotin and P. Kuchment, Band-gap structure of spectra of periodic dielectric and acoustic media. II. Two-dimensional photonic crystals, SIAM J. Appl. Math. 56 (1996), no. 6, 1561-1620, DOI 10.1137/S0036139995285236. MR1417473 (98f:35012)

[143] A. Figotin and P. Kuchment, Spectral properties of classical waves in high-contrast periodic media, SIAM J. Appl. Math. 58 (1998), no. 2, 683-702 (electronic), DOI 10.1137/S0036139996297249. MR1617610 (99f:47061)

[144] N. Filonov, Second-order elliptic equation of divergence form having a compactly supported solution, J. Math. Sci. (New York) 106 (2001), no. 3, 3078-3086, DOI 10.1023/A:1011379807662. Function theory and phase transitions. MR,1906035 (2003h:35047)

[145] N. Filonov, Gaps in the spectrum of the Maxwell operator with periodic coefficients, Comm. Math. Phys. 240 (2003), no. 1-2, 161-170, DOI 10.1007/s00220-003-0904-7. MR2004984 (2004i:35293)

[146] N. Filonov and I. Kachkovskii, On the structure of band edges of $2 d$ periodic elliptic operators, preprint arXiv:1510.04367 (2015).

[147] N. Filonov and F. Klopp, Absolute continuity of the spectrum of a Schrödinger operator with a potential which is periodic in some directions and decays in others, Doc. Math. 9 (2004), 107-121 (electronic). MR2054982(2005i:35045a)

[148] N. Filonov and F. Klopp, Erratum to: "Absolute continuity of the spectrum of a Schrödinger operator with a potential which is periodic in some directions and decays in others" [Doc. Math. 9 (2004), 107-121], Doc. Math. 9 (2004), 135-136. MR2054984(2005i:35045b)

[149] N. Filonov and F. Klopp, Absolutely continuous spectrum for the isotropic Maxwell operator and coefficients that are periodic in some directions and decay in others, Comm. Math. Phys. 258 (2005), no. 1, 75-85, DOI 10.1007/s00220-005-1303-z. MR2166840 (2006f:35197)

[150] N. Filonov and A. V. Sobolev, Absence of the singular continuous component in the spectrum of analytic direct integrals (English, with English and Russian summaries), Zap. Nauchn. 
Sem. S.-Peterburg. Otdel. Mat. Inst. Steklov. (POMI) 318 (2004), no. Kraev. Zadachi Mat. Fiz. i Smezh. Vopr. Teor. Funkts. 36 [35], 298-307, 313, DOI 10.1007/s10958-0060203-x; English transl., J. Math. Sci. (N. Y.) 136 (2006), no. 2, 3826-3831. MR2120804 (2005m:47040)

[151] N. E. Firsova, The Riemann surface of a quasimomentum, and scattering theory for a perturbed Hill operator (Russian), Zap. Naučn. Sem. Leningrad. Otdel. Mat. Inst. Steklov (LOMI) 51 (1975), 183-196, 220. Mathematical questions in the theory of wave propagation, 7. MR0417858 (54 \#5906)

[152] N. E. Firsova, On the global quasimomentum in solid state physics, Mathematical methods in physics (Londrina, 1999), World Sci. Publ., River Edge, NJ, 2000, pp. 98-141. MR 1775625 (2002f:81151)

[153] G. Floquet, Sur les équations différentielles linéaires à coefficients périodiques, Ann. Ecole Norm. 12 (1883), no. 2, 47-89.

[154] G. B. Folland, A course in abstract harmonic analysis, Studies in Advanced Mathematics, CRC Press, Boca Raton, FL, 1995. MR1397028 (98c:43001)

[155] R. L. Frank, On the scattering theory of the Laplacian with a periodic boundary condition. I. Existence of wave operators, Doc. Math. 8 (2003), 547-565 (electronic). MR2029173 (2004k:35286)

[156] R. L. Frank and R. G. Shterenberg, On the scattering theory of the Laplacian with a periodic boundary condition. II. Additional channels of scattering, Doc. Math. 9 (2004), 57-77 (electronic). MR2054980(2005b:35044)

[157] L. Friedlander, On the spectrum of the periodic problem for the Schrödinger operator, Comm. Partial Differential Equations 15 (1990), no. 11, 1631-1647, DOI 10.1080/03605309908820740. MR1079606(92i:35092a)

[158] L. Friedlander, Erratum to: "On the spectrum of the periodic problem for the Schrödinger operator", Comm. Partial Differential Equations 16 (1991), no. 2-3, 527-529. MR1104109 (92i:35092b)

[159] L. Friedlander, On the density of states of periodic media in the large coupling limit, Comm. Partial Differential Equations 27 (2002), no. 1-2, 355-380, DOI 10.1081/PDE-120002790. MR 1886963 (2003d:35194)

[160] L. Friedlander, On the spectrum of a class of second order periodic elliptic differential operators, Comm. Math. Phys. 229 (2002), no. 1, 49-55, DOI 10.1007/s00220-002-0675-6. MR $1917673(2003 \mathrm{k}: 35179)$

[161] L. Friedlander, Absolute continuity of the spectra of periodic waveguides, Waves in periodic and random media (South Hadley, MA, 2002), Contemp. Math. vol. 339, Amer. Math. Soc., Providence, RI, 2003, pp. 37-42, DOI 10.1090/conm/339/06098. MR2042530(2005a:35211)

[162] R. Froese, I. Herbst, M. Hoffmann-Ostenhof, and T. Hoffmann-Ostenhof, $L^{2}$-lower bounds to solutions of one-body Schrödinger equations, Proc. Roy. Soc. Edinburgh Sect. A 95 (1983), no. 1-2, 25-38, DOI 10.1017/S0308210500015778. MR723095(86a:35044)

[163] J. Garnett and E Trubowitz, Gaps and bands of one-dimensional periodic Schrödinger operators, Comment. Math. Helv. 59 (1984), no. 2, 258-312, DOI 10.1007/BF02566350. MR749109(85i:34004)

[164] J. Garnett and E. Trubowitz, Gaps and bands of one-dimensional periodic Schrödinger operators. II, Comment. Math. Helv. 62 (1987), no. 1, 18-37, DOI 10.1007/BF02564436. MR 882963 (88g:34028)

[165] M. Gavrila, Atomic structure and decay in high-frequency fields, in atoms in intense laser fields, Atoms in Intense Laser Fields, Academic Press, 1992, pp. 435-510.

[166] I. M. Gel'fand, Expansion into eigenfunctions of an equation with periodic coefficients, Dokl. Akad. Nauk SSSR (N.S.) 73 (1950), 1117-1120.

[167] C. Gérard, Resonance theory for periodic Schrödinger operators (English, with French summary), Bull. Soc. Math. France 118 (1990), no. 1, 27-54. MR1077086 (91h:35231)

[168] C. Gérard, A proof of the abstract limiting absorption principle by energy estimates, J. Funct. Anal. 254 (2008), no. 11, 2707-2724, DOI 10.1016/j.jfa.2008.02.015. MR 2414218 (2009e:47028)

[169] C. Gérard and F. Nier, The Mourre theory for analytically fibered operators, J. Funct. Anal. 152 (1998), no. 1, 202-219, DOI 10.1006/jfan.1997.3154. MR.1600082 (99b:47030)

[170] C. Gérard and F. Nier, Scattering theory for the perturbations of periodic Schrödinger operators, J. Math. Kyoto Univ. 38 (1998), no. 4, 595-634. MR1669979 (2000e:47023) 
[171] A. Ghoreishi and R. Logan, Positive solutions to a system of periodic parabolic partial differential equations, Differential Integral Equations 9 (1996), no. 3, 607-618. MR.1371711 (97b:35089)

[172] D. Gieseker, H. Knörrer, and E. Trubowitz, An overview of the geometry of algebraic Fermi curves, Algebraic geometry: Sundance 1988, Contemp. Math. vol. 116, Amer. Math. Soc., Providence, RI, 1991, pp. 19-46, DOI 10.1090/conm/116/1108630. MR/1108630(92i:14026)

[173] D. Gieseker, H. Knörrer, and E. Trubowitz, The geometry of algebraic Fermi curves, Perspectives in Mathematics, vol. 14, Academic Press Inc., Boston, MA, 1993. MR.1184395 (95b:14019)

[174] I. M. Glazman, Direct methods of qualitative spectral analysis of singular differential operators, Translated from the Russian by the IPST staff, Israel Program for Scientific Translations, Jerusalem, 1965; Daniel Davey \& Co., Inc., New York, 1966. MR0190800 (32 \#8210)

[175] I. C. Gohberg and M. G. Kreĭn, Introduction to the theory of linear nonselfadjoint operators, Translated from the Russian by A. Feinstein. Translations of Mathematical Monographs, Vol. 18, American Mathematical Society, Providence, R.I. 1969. MR0246142 (39 \#7447)

[176] C. S. Gordon, P. Guerini, T. Kappeler, and D. L. Webb, Inverse spectral results on even dimensional tori (English, with English and French summaries), Ann. Inst. Fourier (Grenoble) 58 (2008), no. 7, 2445-2501. MR.2498357 (2010d:58034)

[177] C. S. Gordon and T. Kappeler, On isospectral potentials on tori, Duke Math. J. 63 (1991), no. 1, 217-233, DOI 10.1215/S0012-7094-91-06310-6. MR1106944(92f:58185)

[178] C. S. Gordon and T. Kappeler, On isospectral potentials on flat tori. II, Comm. Partial Differential Equations 20 (1995), no. 3-4, 709-728, DOI 10.1080/03605309508821109. MR1318086 (96a:58197)

[179] H. Grauert, Analytische Faserungen über holomorph-vollständigen Räumen (German), Math. Ann. 135 (1958), 263-273. MR0098199 (20 \#4661)

[180] H. Grauert and R. Remmert, Theory of Stein spaces, Classics in Mathematics, SpringerVerlag, Berlin, 2004. Translated from the German by Alan Huckleberry; Reprint of the 1979 translation. MR2029201 (2004k:32015)

[181] E. L. Green, Spectral theory of Laplace-Beltrami operators with periodic metrics, ProQuest LLC, Ann Arbor, MI, 1991. Thesis (Ph.D.)-Georgia Institute of Technology. MR2686892

[182] E. L. Green, Spectral theory of Laplace-Beltrami operators with periodic metrics, J. Differential Equations 133 (1997), no. 1, 15-29, DOI 10.1006/jdeq.1996.3204. MR 1426755 (97k:58168)

[183] M. J. Gruber, Noncommutative Bloch theory, J. Math. Phys. 42 (2001), no. 6, 2438-2465, DOI 10.1063/1.1369122. MR 1821870 (2002j:81289)

[184] M. J. Gruber, Measures of Fermi surfaces and absence of singular continuous spectrum for magnetic Schrödinger operators, Math. Nachr. 233/234 (2002), 111127, DOI 10.1002/1522-2616(200201)233:1〈111::AID-MANA111〉3.3.CO;2-L. MR 1879867 (2002m:81049)

[185] M. J. Gruber, Positive measure spectrum for Schrödinger operators with periodic magnetic fields, J. Math. Phys. 44 (2003), no. 4, 1584-1595, DOI 10.1063/1.1556551. MR1963090 (2004f:81072)

[186] V. V. Grushin, Application of the multiparameter theory of perturbations of Fredholm operators to Bloch functions (Russian, with Russian summary), Mat. Zametki 86 (2009), no. 6, 819-828, DOI 10.1134/S0001434609110194; English transl., Math. Notes 86 (2009), no. 5-6, 767-774. MR2643450 (2011b:47032)

[187] V. Guillemin, Spectral theory on $S^{2}$ : some open questions, Adv. in Math. 42 (1981), no. 3, 283-298, DOI 10.1016/0001-8708(81)90043-8. MR642394(83d:58070)

[188] V. Guillemin, Inverse spectral results on two-dimensional tori, J. Amer. Math. Soc. 3 (1990), no. 2, 375-387, DOI 10.2307/1990958. MR1035414 (90m:58214)

[189] R. C. Gunning and H. Rossi, Analytic functions of several complex variables, AMS Chelsea Publishing, Providence, RI, 2009. Reprint of the 1965 original. MR2568219 (2010j:32001)

[190] J. M. Harrison, P. Kuchment, A. Sobolev, and B. Winn, On occurrence of spectral edges for periodic operators inside the Brillouin zone, J. Phys. A 40 (2007), no. 27, 7597-7618, DOI 10.1088/1751-8113/40/27/011. MR2369966(2008j:81039)

[191] W. A. Harrison and M. B. Webb (eds.), The Fermi surface, John Wiley \& Sons, New York, London, 1960 . 
[192] J. E. Heebner, R. W. Boyd, and Q. Park, Scissor solitons and other propagation effects in microresonator modified waveguides, JOSA B 19 (2002), 722-731.

[193] B. Helffer and T. Hoffmann-Ostenhof, Spectral theory for periodic Schrödinger operators with reflection symmetries, Comm. Math. Phys. 242 (2003), no. 3, 501-529, DOI 10.1007/s00220-003-0953-y. MR2020278(2005e:35041)

[194] B. Helffer and A. Mohamed, Asymptotic of the density of states for the Schrödinger operator with periodic electric potential, Duke Math. J. 92 (1998), no. 1, 1-60, DOI 10.1215/S00127094-98-09201-8. MR 1609321 (99e:35166)

[195] R. Hempel and I. Herbst, Bands and gaps for periodic magnetic Hamiltonians, Partial differential operators and mathematical physics (Holzhau, 1994), Oper. Theory Adv. Appl. vol. 78, Birkhäuser, Basel, 1995, pp. 175-184. MR1365330(97d:35158)

[196] R. Hempel and K. Lienau, Spectral properties of periodic media in the large coupling limit, Comm. Partial Differential Equations 25 (2000), no. 7-8, 1445-1470, DOI 10.1080/03605300008821555. MR1765136(2001h:47074)

[197] R. Hempel and O. Post, Spectral gaps for periodic elliptic operators with high contrast: an overview, Progress in analysis, Vol. I, II (Berlin, 2001), World Sci. Publ., River Edge, NJ, 2003, pp. 577-587. MR.2032728 (2004k:35281)

[198] P. Hess, Positive solutions of periodic-parabolic problems and stability, Nonlinear parabolic equations: qualitative properties of solutions (Rome, 1985), Pitman Res. Notes Math. Ser. vol. 149, Longman Sci. Tech., Harlow, 1987, pp. 129-136. MR901101

[199] Y. Higuchi and T. Shirai, Some spectral and geometric properties for infinite graphs, Discrete geometric analysis, Contemp. Math. vol. 347, Amer. Math. Soc., Providence, RI, 2004, pp. 29-56, DOI 10.1090/conm/347/06265. MR2077029 (2006f:47040)

[200] V. Hoang and M. Radosz, Absence of bound states for waveguides in two-dimensional periodic structures, J. Math. Phys. 55 (2014), no. 3, 033506, 20, DOI 10.1063/1.4868480. MR 3221271

[201] J. S. Howland, Scattering theory for Hamiltonians periodic in time, Indiana Univ. Math. J. 28 (1979), no. 3, 471-494, DOI 10.1512/iumj.1979.28.28033. MR529679 (80e:47008)

[202] J. S. Howland, Floquet operators with singular spectrum. I, II (English, with French summary), Ann. Inst. H. Poincaré Phys. Théor. 50 (1989), no. 3, 309-323, 325-334. MR1017967 (91i:81018)

[203] J. S. Howland, Quantum stability, Schrödinger operators (Aarhus, 1991), Lecture Notes in Phys. vol. 403, Springer, Berlin, 1992, pp. 100-122, DOI 10.1007/3-540-55490-4_7. MR 1181243 (94b:81026)

[204] J. S. Howland, Floquet operators with singular spectrum. III (English, with English and French summaries), Ann. Inst. H. Poincaré Phys. Théor. 69 (1998), no. 2, 265-273. MR 1638899 (99i:81037)

[205] V. V. Jikov, S. M. Kozlov, and O. A. Oleĭnik, Homogenization of differential operators and integral functionals, Springer-Verlag, Berlin, 1994. Translated from the Russian by G. A. Yosifian [G. A. Iosif'yan]. MR1329546 (96h:35003b)

[206] J. D. Joannopoulos, S. Johnson, R. D. Meade, and J. N. Winn, Photonic crystals: Molding the flow of light, 2nd ed., Princeton University Press, Princeton, N.J., 2008.

[207] I. Kachkovskil and N. Filonov, Absolute continuity of the spectrum of a periodic Schrödinger operator in a multidimensional cylinder (Russian, with Russian summary), Algebra i Analiz 21 (2009), no. 1, 133-152, DOI 10.1090/S1061-0022-09-01087-5; English transl., St. Petersburg Math. J. 21 (2010), no. 1, 95-109. MR2553054(2010i:35054)

[208] I. Kachkovskiu and N. Filonov, Absolute continuity of the spectrum of the periodic Schrödinger operator in a layer and in a smooth cylinder (Russian, with English and Russian summaries), Zap. Nauchn. Sem. S.-Peterburg. Otdel. Mat. Inst. Steklov. (POMI) 385 (2010), no. Kraevye Zadachi Matematicheskoi Fiziki i Smezhnye Voprosy Teorii Funktsii. 41, 69-81, 235, DOI 10.1007/s10958-011-0547-8; English transl., J. Math. Sci. (N. Y.) 178 (2011), no. 3, 274-281. MR2749370 (2011k:35032)

[209] T. Kappeler, On isospectral periodic potentials on a discrete lattice. I, Duke Math. J. 57 (1988), no. 1, 135-150, DOI 10.1215/S0012-7094-88-05705-5. MR952228 (90b:39010a)

[210] T. Kappeler, On isospectral potentials on a discrete lattice. II, Adv. in Appl. Math. 9 (1988), no. 4, 428-438, DOI 10.1016/0196-8858(88)90021-8. MR968676 (90b:39010b)

[211] T. Kappeler, Isospectral potentials on a discrete lattice. III, Trans. Amer. Math. Soc. 314 (1989), no. 2, 815-824, DOI 10.2307/2001410. MR961624(90b:39010c) 
[212] T. Kappeler, B. Schaad, and P. Topalov, Asymptotics of spectral quantities of Schrödinger operators, Spectral geometry, Proc. Sympos. Pure Math. vol. 84, Amer. Math. Soc., Providence, RI, 2012, pp. 243-284, DOI 10.1090/pspum/084/1360. MR2985321

[213] Y. E. Karpeshina, Perturbation theory for the Schrödinger operator with a periodic potential, Lecture Notes in Mathematics, vol. 1663, Springer-Verlag, Berlin, 1997. MR.1472485 (2000i:35002)

[214] Y. E. Karpeshina, On the density of states for the periodic Schrödinger operator, Ark. Mat. 38 (2000), no. 1, 111-137, DOI 10.1007/BF02384494. MR1749362(2001g:47088)

[215] Y. Karpeshina, Spectral properties of the periodic magnetic Schrödinger operator in the high-energy region. Two-dimensional case, Comm. Math. Phys. 251 (2004), no. 3, 473-514, DOI 10.1007/s00220-004-1129-0. MR2102328(2005m:81096)

[216] Y. Karpeshina and Y.-R. Lee, Absolutely continuous spectrum of a polyharmonic operator with a limit periodic potential in dimension two, Comm. Partial Differential Equations 33 (2008), no. 7-9, 1711-1728, DOI 10.1080/03605300802289220. MR2450178 (2009g:35046)

[217] T. Kato, Perturbation theory for linear operators, Classics in Mathematics, Springer-Verlag, Berlin, 1995. Reprint of the 1980 edition. MR1335452 (96a:47025)

[218] M. I. Katsnelson, Graphene: carbon in two dimensions, Cambridge Univ. Press, 2012.

[219] M. Kha, Green's function asymptotics of periodic elliptic operators on abelian coverings of compact manifolds, submitted, preprint arXiv: 1511.00276.

[220] M. Kha, P. Kuchment, and A. Raich, Green's function asymptotics near the internal edges of spectra of periodic elliptic operators: spectral gap interior, J. Spectral Theory (to appear), arXiv:1508.06703, (2015).

[221] A. Khrabustovskyi, Periodic elliptic operators with asymptotically preassigned spectrum, Asymptot. Anal. 82 (2013), no. 1-2, 1-37. MR3088339

[222] A. Khrabustovskyi, Opening up and control of spectral gaps of the Laplacian in periodic domains, J. Math. Phys. 55 (2014), no. 12, 121502, 23, DOI 10.1063/1.4902935. MR3390523

[223] A. Khrabustovskyi and E. Khruslov, Gaps in the spectrum of the Neumann Laplacian generated by a system of periodically distributed traps, Math. Methods Appl. Sci. 38 (2015), no. 1, 11-26, DOI 10.1002/mma.3046. MR3291299

[224] W. Kirsch and B. Simon, Comparison theorems for the gap of Schrödinger operators, J. Funct. Anal. 75 (1987), no. 2, 396-410, DOI 10.1016/0022-1236(87)90103-0. MR916759 (89b:35127)

[225] C. Kittel, Introduction to solid state physics, Wiley, New York, 1976.

[226] A. Klein, A. Koines, and M. Seifert, Generalized eigenfunctions for waves in inhomogeneous media, J. Funct. Anal. 190 (2002), no. 1, 255-291, DOI 10.1006/jfan.2001.3887. Special issue dedicated to the memory of I. E. Segal. MR 1895534 (2004a:35165)

[227] F. Klopp, Absolute continuity of the spectrum of a Landau Hamiltonian perturbed by a generic periodic potential, Math. Ann. 347 (2010), no. 3, 675-687, DOI 10.1007/s00208009-0452-3. MR2640047 (2011g:47106)

[228] F. Klopp and J. Ralston, Endpoints of the spectrum of periodic operators are generically simple (English, with English and French summaries), Methods Appl. Anal. 7 (2000), no. 3, 459-463. Cathleen Morawetz: a great mathematician. MR 1869296 (2002i:47055)

[229] H. Knörrer and E. Trubowitz, A directional compactification of the complex Bloch variety, Comment. Math. Helv. 65 (1990), no. 1, 114-149, DOI 10.1007/BF02566598. MR1036133 (91k:58134)

[230] T. Kobayashi, K. Ono, and T. Sunada, Periodic Schrödinger operators on a manifold, Forum Math. 1 (1989), no. 1, 69-79, DOI 10.1515/form.1989.1.69. MR978976 (89k:58288)

[231] W. Kohn, Analytic properties of Bloch waves and Wannier functions, Phys. Rev. (2) 115 (1959), 809-821. MR0108284 (21 \#7000)

[232] Y. A. Kordyukov, Spectral gaps for periodic Schrödinger operators with strong magnetic fields, Comm. Math. Phys. 253 (2005), no. 2, 371-384, DOI 10.1007/s00220-004-1134-3. MR2140253 (2006h:81079)

[233] Y. A. Kordyukov, Semiclassical asymptotics and spectral gaps for periodic magnetic Schrödinger operators on covering manifolds, $C^{*}$-algebras and elliptic theory, Trends Math. Birkhäuser, Basel, 2006, pp. 129-150, DOI 10.1007/978-3-7643-7687-1_6. MR2276917 (2008f:58034) 
[234] E. Korotyaev and I. Lobanov, Schrödinger operators on zigzag nanotubes, Ann. Henri Poincaré 8 (2007), no. 6, 1151-1176, DOI 10.1007/s00023-007-0331-y. MR.2355344 (2008g:81076)

[235] E. Korotyaev and A. Pushnitski, On the high-energy asymptotics of the integrated density of states, Bull. London Math. Soc. 35 (2003), no. 6, 770-776, DOI 10.1112/S0024609303002467. MR 2000023(2004h:35046)

[236] S. G. Krantz and H. R. Parks, A primer of real analytic functions, 2nd ed. Birkhäuser Advanced Texts: Basler Lehrbücher. [Birkhäuser Advanced Texts: Basel Textbooks], Birkhäuser Boston, Inc., Boston, MA, 2002. MR.1916029 (2003f:26045)

[237] K. Krupchyk and G. Uhlmann, Absolute continuity of the periodic schrödinger operator in transversal geometry, arXiv:1312.2989.

[238] P. Kuchment, Representations of solutions of invariant differential equations on some symmetric spaces (Russian), Dokl. Akad. Nauk SSSR 259 (1981), no. 3, 532-535. MR625758 (82i:43008)

[239] P. Kuchment, Floquet theory for partial differential equations (Russian), Uspekhi Mat. Nauk 37 (1982), no. 4(226), 3-52, 240. MR667973 (84b:35018)

[240] P. Kuchment, Floquet theory for partial differential equations (Russian), Uspekhi Mat. Nauk 37 (1982), no. 4(226), 3-52, 240. MR667973(84b:35018)

[241] P. Kuchment, Spectral synthesis in spaces of solutions of differential equations invariant with respect to groups of transformations (Russian), Application of topology in modern analysis (Russian), Novoe Global. Anal. Voronezh. Gos. Univ., Voronezh, 1985, pp. 87-105, 176. MR831671 (87e:58216)

[242] P. Kuchment, Spherical representation of solutions of invariant differential equations on a Riemannian symmetric space of noncompact type (Russian), Izv. Akad. Nauk SSSR Ser. Mat. 49 (1985), no. 6, 1260-1273, 1343. MR816856 (87d:58157)

[243] P. Kuchment, On the Floquet theory of periodic difference equations, Geometrical and algebraical aspects in several complex variables (Cetraro, 1989), Sem. Conf. vol. 8, EditEl, Rende, 1991, pp. 201-209. MR.1222215 (94k:39001)

[244] P. Kuchment, Floquet theory for partial differential equations, Operator Theory: Advances and Applications, vol. 60, Birkhäuser Verlag, Basel, 1993. MR.1232660 (94h:35002)

[245] P. Kuchment, The mathematics of photonic crystals, Mathematical modeling in optical science, Frontiers Appl. Math. vol. 22, SIAM, Philadelphia, PA, 2001, pp. 207-272. MR 1831334 (2002k:78002)

[246] P. Kuchment, Quantum graphs. II. Some spectral properties of quantum and combinatorial graphs, J. Phys. A 38 (2005), no. 22, 4887-4900, DOI 10.1088/0305-4470/38/22/013. MR2148631(2006a:81035)

[247] P. Kuchment, Integral representations of solutions of periodic elliptic equations, Probability and mathematical physics, CRM Proc. Lecture Notes, vol. 42, Amer. Math. Soc., Providence, RI, 2007, pp. 323-339. MR 2352277 (2008k:35077)

[248] P. Kuchment, On Sunada's no gap conjecture, unpublished (2007).

[249] P. Kuchment, Tight frames of exponentially decaying Wannier functions, J. Phys. A 42 (2009), no. 2, 025203, 16, DOI 10.1088/1751-8113/42/2/025203. MR2525287(2011d:81103)

[250] P. Kuchment, Introduction to periodic operators, video at http://www.newton.ac.uk/event/ pepw01/timetable, 2015.

[251] P. Kuchment and L. A. Kunyansky, Spectral properties of high contrast band-gap materials and operators on graphs, Experiment. Math. 8 (1999), no. 1, 1-28. MR1685034 (2000c:78027)

[252] P. Kuchment and S. Levendorskiî, On the structure of spectra of periodic elliptic operators, Trans. Amer. Math. Soc. 354 (2002), no. 2, 537-569, DOI 10.1090/S0002-9947-01-02878-1. MR 1862558 (2002g:35163)

[253] P. Kuchment and Y. Pinchover, Integral representations and Liouville theorems for solutions of periodic elliptic equations, J. Funct. Anal. 181 (2001), no. 2, 402-446, DOI 10.1006/jfan.2000.3727. MR.1821702 (2001m:35067)

[254] P. Kuchment and Y. Pinchover, Liouville theorems and spectral edge behavior on abelian coverings of compact manifolds, Trans. Amer. Math. Soc. 359 (2007), no. 12, 5777-5815, DOI 10.1090/S0002-9947-07-04196-7. MR2336306(2008h:58037)

[255] P. Kuchment and O. Post, On the spectra of carbon nano-structures, Comm. Math. Phys. 275 (2007), no. 3, 805-826, DOI 10.1007/s00220-007-0316-1. MR2336365 (2008j:81041) 
[256] P. Kuchment and A. Raich, Green's function asymptotics near the internal edges of spectra of periodic elliptic operators. Spectral edge case, Math. Nachr. 285 (2012), no. 14-15, 18801894, DOI 10.1002/mana.201100272. MR2988010

[257] P. Kuchment and B. Vainberg, On embedded eigenvalues of perturbed periodic Schrödinger operators, Spectral and scattering theory (Newark, DE, 1997), Plenum, New York, 1998, pp. 67-75. MR1625271 (99e:35032)

[258] P. Kuchment and B. Vainberg, On absence of embedded eigenvalues for Schrödinger operators with perturbed periodic potentials, Comm. Partial Differential Equations 25 (2000), no. 9-10, 1809-1826, DOI 10.1080/03605300008821568. MR.1778781(2001i:47074)

[259] P. Kuchment and B. Vainberg, On the structure of eigenfunctions corresponding to embedded eigenvalues of locally perturbed periodic graph operators, Comm. Math. Phys. 268 (2006), no. 3, 673-686, DOI 10.1007/s00220-006-0105-2. MR2259210(2007h:39034)

[260] P. Kuchment and L. Zelenko, On the floquet representations of exponentially increasing solutions of elliptic equations with periodic coefficients, Soviet Math. Dokl. 19 (1978), no. 2, 506-507.

[261] I. S. Lapin, One version of the Bethe-Sommerfeld conjecture, J. Math. Sci. (N. Y.) 117 (2003), no. 3, 4157-4166, DOI 10.1023/A:1024812419240. Nonlinear problems and function theory. MR2027452 (2004k:81114)

[262] P. D. Lax, Periodic solutions of the KdV equation, Comm. Pure Appl. Math. 28 (1975), 141-188. MR0369963 (51 \#6192)

[263] M. Lee, Conic dispersion surfaces for point scatterers on a honeycomb lattice, 2014, arXiv: 1402.5179.

[264] S. Z. Levendorskiı̆, Magnetic Floquet theory and spectral asymptotics for Schrödinger operators (Russian), Funktsional. Anal. i Prilozhen. 30 (1996), no. 4, 77-80, DOI 10.1007/BF02509625; English transl., Funct. Anal. Appl. 30 (1996), no. 4, 282-285 (1997). MR, 1444468 (98d:35169)

[265] S. Z. Levendorskiı, Floquet's theory for the Schrödinger operator with perturbed periodic potential, and exact spectral asymptotics (Russian), Dokl. Akad. Nauk 355 (1997), no. 1, 21-24. MR 1482093 (99b:47067)

[266] P. Li, Curvature and function theory on Riemannian manifolds, Surveys in differential geometry, Surv. Differ. Geom., VII, Int. Press, Somerville, MA, 2000, pp. 375-432, DOI 10.4310/SDG.2002.v7.n1.a13. MR1919432(2003g:53047)

[267] P. Li, Geometric analysis, Cambridge Studies in Advanced Mathematics, vol. 134, Cambridge University Press, Cambridge, 2012. MR2962229

[268] V. B. Lidskii, Eigenfunction expansions for equations with periodic coefficients, Appendix VIII to the Russian (1961) edition of Titchmarsh' book.

[269] V. Ya. Lin and Y. Pinchover, Manifolds with group actions and elliptic operators, Mem. Amer. Math. Soc. 112 (1994), no. 540, vi+78, DOI 10.1090/memo/0540. MR 1230774 (95d:58119)

[270] F. Lledó and O. Post, Existence of spectral gaps, covering manifolds and residually finite groups, Rev. Math. Phys. 20 (2008), no. 2, 199-231, DOI 10.1142/S0129055X08003286. MR2400010(2008m:58072)

[271] S. Łojasiewicz, Stratification des ensembles analytiques avec les propriétés (A) et (B) de Whitney (French), Fonctions analytiques de plusieurs variables et analyse complexe (Colloq. Internat. C.N.R.S. No. 208, Paris, 1972), Gauthier-Villars, Paris, 1974, pp. 116-130. "Agora Mathematica", No. 1. MR0457772 (56 \#15976)

[272] S. Łojasiewicz, Introduction to complex analytic geometry, Birkhäuser Verlag, Basel, 1991. Translated from the Polish by Maciej Klimek. MR.1131081 (92g:32002)

[273] A. M. Lyapunov, Sur une série relative a la theorie des equations differentielles lineaires a coefficients periodiques, Compt. Rend. 123 (1896), no. 26, 1248-1252.

[274] A. M. Lyapunov, Sur une equation transcendante et les equations differentielles lineaires du second ordre a coefficients periodiques, Compt. Rend. 128 (1899), no. 18, 1085-1088.

[275] W. Magnus and S. Winkler, Hill's equation, Interscience Tracts in Pure and Applied Mathematics, No. 20, Interscience Publishers John Wiley \& Sons New York-London-Sydney, 1966. MR0197830 (33 \#5991)

[276] V. A. Marčenko and I. V. Ostrovs'kiü, A characterization of the spectrum of the Hill operator (Russian), Mat. Sb. (N.S.) 97(139) (1975), no. 4(8), 540-606, 633-634. MR0409965 (53 \#13717) 


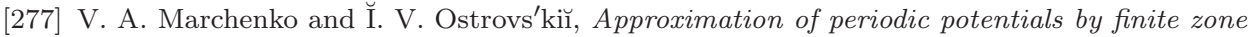
potentials (Russian), Vestnik Khar'kov. Gos. Univ. 205 (1980), 4-40, 139. MR643352 (84b:34032)

[278] V. A. Marchenko and I. V. Ostrovsky, Corrections to the article: "Approximation of periodic by finite-zone potentials" [Selecta Math. Soviet. 6 (1987), no. 2, 101-136; see MR0910538 (88f:00011)], Selecta Math. Soviet. 7 (1988), no. 1, 99-100. Selected translations. MR967083 $(89 \mathrm{j}: 34033)$

[279] N. Marzari, I Souza, I., and D. Vanderbilt, An introduction to maximally-localized wannier functions, Highlight of the Month, Psi-K Newsletter 57 (2003), no. 4, 129-168.

[280] N. Marzari and D. Vanderbilt, Maximally localized generalized wannier functions for composite energy bands, Phys. Rev. B 56 (1997, NUMBER = 20, PAGES = 12847-12865,).

[281] V. Mathai and M. Shubin, Semiclassical asymptotics and gaps in the spectra of magnetic Schrödinger operators, Proceedings of the Euroconference on Partial Differential Equations and their Applications to Geometry and Physics (Castelvecchio Pascoli, 2000), Geom. Dedicata 91 (2002), 155-173, DOI 10.1023/A:1016245930716. MR.1919898(2004f:58040)

[282] H. P. McKean and E. Trubowitz, Hill's operator and hyperelliptic function theory in the presence of infinitely many branch points, Comm. Pure Appl. Math. 29 (1976), no. 2, 143226. MR0427731 (55 \#761)

[283] H. P. McKean and P. van Moerbeke, The spectrum of Hill's equation, Invent. Math. 30 (1975), no. 3, 217-274. MR0397076 (53 \#936)

[284] V. Z. Meshkov, On the possible rate of decrease at infinity of the solutions of second-order partial differential equations (Russian), Mat. Sb. 182 (1991), no. 3, 364-383; English transl., Math. USSR-Sb. 72 (1992), no. 2, 343-361. MR1110071(92d:35032)

[285] A. I. Miloslavskiı̌, Floquet solutions, and the reducibility of a certain class of differential equations in a Banach space (Russian), Izv. Severo-Kavkaz. Naučn. Centra Vysš. Školy Ser. Estestv. Nauk. 4 (1975), 82-87, 118. MR0470395 (57 \#10149)

[286] A. I. Miloslavskiur, The decay of the solutions of an abstract parabolic equation with a periodic operator coefficient (Russian), Izv. Severo-Kavkaz. Naučn. Centra Vysš. Školy Ser. Estestv. Nauk. 2 (1976), 12-15, 116. MR0435541 (55 \#8500)

[287] A. I. Miloslavskiu, Floquet theory for abstract parabolic equations with periodic coefficients, Ph.D. thesis, Rostov State University. Russia, 1976.

[288] A. I. Miloslavskiı̌, On the Floquet theory for parabolic equations (Russian), Funkcional. Anal. i Priložen. 10 (1976), no. 2, 80-81. MR0473390 (57 \#13057)

[289] A. I. Miloslavskiǔ, On the Floquet theory for parabolic equations, Teor. Funktsiü Funktsional. Anal. i Prilozhen. (1979), no. 31, 98-102, 168. MR.548296 (80k:34086)

[290] A. I. Miloslavskiŭ, Asymptotic behavior of the solutions of abstract integro-differential equations with periodic coefficients (Russian), Funktsional. Anal. i Prilozhen. 22 (1988), no. 3, 77-78, DOI 10.1007/BF01077633; English transl., Funct. Anal. Appl. 22 (1988), no. 3, 234235 (1989). MR.961767(89j:45007)

[291] A. I. Miloslavskiı̌, An abstract integro-differential equation with a periodic coefficient. I (Russian), Teor. Funktsǐ Funktsional. Anal. i Prilozhen. 53 (1990), 100-108, DOI 10.1007/BF01109699; English transl., J. Soviet Math. 58 (1992), no. 6, 563-568. MR 1077229 (91k:47126a)

[292] A. I. Miloslavskiŭ, An abstract integro-differential equation with a periodic coefficient. II (Russian), Teor. Funktsiŭ Funktsional. Anal. i Prilozhen. 54 (1990), 57-68, DOI 10.1007/BF01097285; English transl., J. Soviet Math. 58 (1992), no. 4, 333-342. MR 1080726 $(91 \mathrm{k}: 47126 \mathrm{~b})$

[293] B. Mityagin, The zero set of a real analytic function, arXiv:1512.07276 (2015).

[294] A. Mohamed, Asymptotic of the density of states for the Schrödinger operator with periodic electromagnetic potential, J. Math. Phys. 38 (1997), no. 8, 4023-4051, DOI 10.1063/1.532105. MR1459642(99d:81036)

[295] A. Morame, The absolute continuity of the spectrum of Maxwell operator in a periodic media, J. Math. Phys. 41 (2000), no. 10, 7099-7108, DOI 10.1063/1.1288794. MR.1781426 (2001h:82103)

[296] S. Morozov, L. Parnovski, and I. Pchelintseva, Lower bound on the density of states for periodic Schrödinger operators, Operator theory and its applications, Amer. Math. Soc. Transl. Ser. 2, vol. 231, Amer. Math. Soc., Providence, RI, 2010, pp. 161-171. MR2757529 (2012e:35179) 
[297] S. Morozov, L. Parnovski, and I. Pchelintseva, Lower bound on the density of states for periodic Schrödinger operators, Operator theory and its applications, Amer. Math. Soc. Transl. Ser. 2, vol. 231, Amer. Math. Soc., Providence, RI, 2010, pp. 161-171. MR 2757529 (2012e:35179)

[298] S. Morozov, L. Parnovski, and R. Shterenberg, Complete asymptotic expansion of the integrated density of states of multidimensional almost-periodic pseudo-differential operators, Ann. Henri Poincaré 15 (2014), no. 2, 263-312, DOI 10.1007/s00023-013-0246-8. MR 3159982

[299] J. Moser and M. Struwe, On a Liouville-type theorem for linear and nonlinear elliptic differential equations on a torus, Bol. Soc. Brasil. Mat. (N.S.) 23 (1992), no. 1-2, 1-20, DOI 10.1007/BF02584809. MR 1203171 (94b:35051)

[300] M. Murata and T. Tsuchida, Asymptotics of Green functions and Martin boundaries for elliptic operators with periodic coefficients, J. Differential Equations 195 (2003), no. 1, 82118, DOI 10.1016/S0022-0396(03)00192-X. MR2019244(2004k:35067)

[301] M. Murata and T. Tsuchida, Asymptotics of Green functions and the limiting absorption principle for elliptic operators with periodic coefficients, J. Math. Kyoto Univ. 46 (2006), no. 4, 713-754. MR2320348(2008d:35002)

[302] G. Nenciu, Existence of the exponentially localised Wannier functions, Comm. Math. Phys. 91 (1983), no. 1, 81-85. MR719812 (84j:81146)

[303] O. A. Nielsen, Direct integral theory, Lecture Notes in Pure and Applied Mathematics, vol. 61, Marcel Dekker, Inc., New York, 1980. MR591683(82e:46081)

[304] S. P. Novikov, Bloch functions in the magnetic field and vector bundles. Typical dispersion relations and their quantum numbers (Russian), Dokl. Akad. Nauk SSSR 257 (1981), no. 3, 538-543. MR610347 (82h:81111)

[305] S. P. Novikov, Two-dimensional Schrödinger operators in periodic fields (Russian), Current problems in mathematics, Vol. 23, Itogi Nauki i Tekhniki, Akad. Nauk SSSR, Vsesoyuz. Inst. Nauchn. i Tekhn. Inform., Moscow, 1983, pp. 3-32. MR734312 (85i:81020)

[306] S. P. Novikov, Solitons and geometry, Lezioni Fermiane. [Fermi Lectures], Published for the Scuola Normale Superiore, Pisa; by Cambridge University Press, Cambridge, 1994. MR 1272686 (95i:58091)

[307] S. Novikov, S. V. Manakov, L. P. Pitaevskiı̌, and V. E. Zakharov, Theory of solitons, Contemporary Soviet Mathematics, Consultants Bureau [Plenum], New York, 1984. The inverse scattering method; Translated from the Russian. MR779467 (86k:35142)

[308] F. Odeh and J. B. Keller, Partial differential equations with periodic coefficients and Bloch waves in crystals, J. Mathematical Phys. 5 (1964), 1499-1504. MR0168924 (29 \#6180)

[309] B. S. Ong, Spectral problems of optical waveguides and quantum graphs, ProQuest LLC, Ann Arbor, MI, 2006. Thesis (Ph.D.)-Texas A\&M University. MR2709215

[310] V. P. Palamodov, Lineinye differentsialnye operatory s postoyaannymi koeffitsientami (Russian), Izdat. "Nauka", Moscow, 1967. MR0243193 (39 \#4517)

[311] V. P. Palamodov, Linear differential operators with constant coefficients, Translated from the Russian by A. A. Brown. Die Grundlehren der mathematischen Wissenschaften, Band 168, Springer-Verlag, New York-Berlin, 1970. MR0264197 (41 \#8793)

[312] V. P. Palamodov, Harmonic synthesis of solutions of elliptic equation with periodic coefficients (English, with English and French summaries), Ann. Inst. Fourier (Grenoble) 43 (1993), no. 3, 751-768. MR 1242614 (95f:35037)

[313] G. Panati, Triviality of Bloch and Bloch-Dirac bundles, Ann. Henri Poincaré 8 (2007), no. 5, 995-1011, DOI 10.1007/s00023-007-0326-8. MR2342883 (2008d:81074)

[314] G. Panati and A. Pisante, Bloch bundles, Marzari-Vanderbilt functional and maximally localized Wannier functions, Comm. Math. Phys. 322 (2013), no. 3, 835-875, DOI 10.1007/s00220-013-1741-y. MR3079333

[315] V. Papanicolaou, Private communication.

[316] V. Papanicolaou, Some results on ordinary differential operators with periodic coefficients, Complex Analysis and Operator Theory 9 (2015), no. 7.

[317] L. Parnovski, Bethe-Sommerfeld conjecture, Ann. Henri Poincaré 9 (2008), no. 3, 457-508, DOI 10.1007/s00023-008-0364-x. MR2419769 (2009f:81068)

[318] L. Parnovski and R. Shterenberg, Asymptotic expansion of the integrated density of states of a two-dimensional periodic Schrödinger operator, Invent. Math. 176 (2009), no. 2, 275-323, DOI 10.1007/s00222-008-0164-4. MR2495765(2010d:35048) 
[319] L. Parnovski and R. Shterenberg, Asymptotic expansion of the integrated density of states of a two-dimensional periodic Schrödinger operator, Invent. Math. 176 (2009), no. 2, 275-323, DOI 10.1007/s00222-008-0164-4. MR2495765 (2010d:35048)

[320] L. Parnovski and R. Shterenberg, Complete asymptotic expansion of the integrated density of states of multidimensional almost-periodic Schrödinger operators, Ann. of Math. (2) 176 (2012), no. 2, 1039-1096, DOI 10.4007/annals.2012.176.2.8. MR2950770

[321] L. Parnovski and N. Sidorova, Critical dimensions for counting lattice points in Euclidean annuli, Math. Model. Nat. Phenom. 5 (2010), no. 4, 293-316, DOI 10.1051/mmnp/20105413. MR2662460 (2011e:11162)

[322] L. Parnovski and A. V. Sobolev, On the Bethe-Sommerfeld conjecture, Journées "Équations aux Dérivées Partielles" (La Chapelle sur Erdre, 2000), Univ. Nantes, Nantes, 2000, pp. Exp. No. XVII, 13. MR1775693(2002i:35137)

[323] L. Parnovski and A. V. Sobolev, Bethe-Sommerfeld conjecture for periodic operators with strong perturbations, Invent. Math. 181 (2010), no. 3, 467-540, DOI 10.1007/s00222-0100251-1. MR2660451 (2011f:35242)

[324] L. Parnovskii and R. Shterenberg, Personal communication, Unpublished (2015).

[325] B. S. Pavlov, The theory of extensions, and explicitly solvable models (Russian), Uspekhi Mat. Nauk 42 (1987), no. 6(258), 99-131, 247. MR933997 (89b:47009)

[326] Y. Pinchover, On positive solutions of elliptic equations with periodic coefficients in unbounded domains, Maximum principles and eigenvalue problems in partial differential equations (Knoxville, TN, 1987), Pitman Res. Notes Math. Ser. vol. 175, Longman Sci. Tech., Harlow, 1988, pp. 218-230. MR963470 (89k:35021)

[327] Y. Pinchover, On positive solutions of second-order elliptic equations, stability results, and classification, Duke Math. J. 57 (1988), no. 3, 955-980, DOI 10.1215/S0012-7094-88-057432. MR.975130 (90c:35023)

[328] R. G. Pinsky, Second order elliptic operators with periodic coefficients: criticality theory, perturbations, and positive harmonic functions, J. Funct. Anal. 129 (1995), no. 1, 80-107, DOI 10.1006/jfan.1995.1043. MR.1322643(96b:35038)

[329] A. Pliś, Non-uniqueness in Cauchy's problem for differential equations of elliptic type, J. Math. Mech. 9 (1960), 557-562. MR0121568 (22 \#12305)

[330] V. N. Popov and M. M. Skriganov, Remark on the structure of the spectrum of a twodimensional Schrödinger operator with periodic potential (Russian, with English summary), Zap. Nauchn. Sem. Leningrad. Otdel. Mat. Inst. Steklov. (LOMI) 109 (1981), 131-133, 181, 183-184. Differential geometry, Lie groups and mechanics, IV. MR629118 (83a:35074)

[331] O. Post, Periodic manifolds with spectral gaps, J. Differential Equations 187 (2003), no. 1, 23-45, DOI 10.1016/S0022-0396(02)00006-2. MR1946544(2003m:58047)

[332] M. Reed and B. Simon, Methods of modern mathematical physics. I, 2nd ed. Academic Press, Inc. [Harcourt Brace Jovanovich, Publishers], New York, 1980. Functional analysis. MR751959(85e:46002)

[333] O. Reingold, S. Vadhan, and A. Wigderson, Entropy waves, the zig-zag graph product, and new constant-degree expanders, Ann. of Math. (2) 155 (2002), no. 1, 157-187, DOI 10.2307/3062153. MR 1888797(2003c:05145)

[334] F. S. Rofe-Beketov, On the spectrum of non-selfadjoint differential operators with periodic coefficients (Russian), Dokl. Akad. Nauk SSSR 152 (1963), 1312-1315. MR0157033 $(28$ \#274)

[335] F. S. Rofe-Beketov, A finiteness test for the number of discrete levels which can be introduced into the gaps of the continuous spectrum by perturbations of a periodic potential (Russian), Dokl. Akad. Nauk SSSR 156 (1964), 515-518. MR0160967 (28 \#4176)

[336] F. S. Rofe-Beketov, A perturbation of a Hill's operator, that has a first moment and a non-zero integral, introduces a single discrete level into each of the distant spectral lacunae (Russian), Mathematical physics and functional analysis, No. 4 (Russian), Fiz.-Tehn. Inst. Nizkih Temperatur, Akad. Nauk Ukrain. SSR, Kharkov, 1973, pp. 158-159, 163. MR0477257 (57 \#16798)

[337] F. S. Rofe-Beketov, Spectrum perturbations, the knezer-type constants and the effective mass of zones-type potentials, Constructive Theory of Functions 1984 (Sofia), 1984, Proceedings of the International Conference on Constructive Function Theory, Varna 1984, pp. 757-766. 
[338] B. Scarpellini, Stability, instability, and direct integrals, Chapman \& Hall/CRC Research Notes in Mathematics, vol. 402, Chapman \& Hall/CRC, Boca Raton, FL, 1999. MR.1682260 (2000j:35023)

[339] J. H. Schenker and M. Aizenman, The creation of spectral gaps by graph decoration, Lett. Math. Phys. 53 (2000), no. 3, 253-262, DOI 10.1023/A:1011032212489. MR1808253 (2001k:47008)

[340] Z. Shen, Absolute continuity of generalized periodic Schrödinger operators, Harmonic analysis and boundary value problems (Fayetteville, AR, 2000), Contemp. Math. vol. 277, Amer. Math. Soc., Providence, RI, 2001, pp. 113-126, DOI 10.1090/conm/277/04541. MR1840430 (2002j:35078)

[341] Z. Shen, On absolute continuity of the periodic Schrödinger operators, Internat. Math. Res. Notices (2001), no. 1, 1-31. MR1809495 (2002a:47078)

[342] Z. Shen, On the Bethe-Sommerfeld conjecture for higher-order elliptic operators, Math. Ann. 326 (2003), no. 1, 19-41, DOI 10.1007/s00208-003-0395-z. MR1981610 (2004c:35299)

[343] Z. Shen and P. Zhao, Uniform Sobolev inequalities and absolute continuity of periodic operators, Trans. Amer. Math. Soc. 360 (2008), no. 4, 1741-1758, DOI 10.1090/S0002-994707-04545-X. MR2366961 (2010j:35095)

[344] D. Shenk and M. A. Shubin, Asymptotic expansion of state density and the spectral function of the Hill operator (Russian), Mat. Sb. (N.S.) 128(170) (1985), no. 4, 474-491, 575. MR 820398 (87h:34078)

[345] S. P. Shipman, Eigenfunctions of unbounded support for embedded eigenvalues of locally perturbed periodic graph operators, Comm. Math. Phys. 332 (2014), no. 2, 605-626, DOI 10.1007/s00220-014-2113-y. MR3257657

[346] È. È. Shnol', On the behavior of the eigenfunctions of Schrödinger's equation, Mat. Sb. (N.S.) 42 (84) (1957), no. 3, 273-286. MR0125315 (23:A2618)

[347] R. G. Shterenberg, Absolute continuity of a two-dimensional magnetic periodic Schrödinger operator with electric potential of measure derivative type (Russian, with English and Russian summaries), Zap. Nauchn. Sem. S.-Peterburg. Otdel. Mat. Inst. Steklov. (POMI) 271 (2000), no. Kraev. Zadachi Mat. Fiz. i Smezh. Vopr. Teor. Funkts. 31, 276-312, 318, DOI 10.1023/A:1023334206109; English transl., J. Math. Sci. (N. Y.) 115 (2003), no. 6, 28622882. MR $1810620(2002 \mathrm{~m}: 35171)$

[348] R. G. Shterenberg, The Schrödinger operator in a periodic waveguide on a plane and quasiconformal mappings (Russian, with English and Russian summaries), Zap. Nauchn. Sem. S.-Peterburg. Otdel. Mat. Inst. Steklov. (POMI) 295 (2003), no. Kraev. Zadachi Mat. Fiz. i Smezh. Vopr. Teor. Funkts. 33, 204-243, 247, DOI 10.1007/s10958-005-0152-9; English transl., J. Math. Sci. (N. Y.) 127 (2005), no. 2, 1936-1956. MR1983118 (2004e:78030)

[349] R. G. Shterenberg, An example of a periodic magnetic Schrödinger operator with a degenerate lower edge of the spectrum (Russian, with Russian summary), Algebra i Analiz 16 (2004), no. 2, 177-185, DOI 10.1090/S1061-0022-05-00858-7; English transl., St. Petersburg Math. J. 16 (2005), no. 2, 417-422. MR2068347 (2005d:35220)

[350] R. G. Shterenberg, On the structure of the lower edge of the spectrum of a periodic magnetic Schrödinger operator with small magnetic potential (Russian, with Russian summary), Algebra i Analiz 17 (2005), no. 5, 232-243, DOI 10.1090/S1061-0022-06-00933-2; English transl., St. Petersburg Math. J. 17 (2006), no. 5, 865-873. MR2241429 (2007f:35046)

[351] T. A. Suslina and R. G. Shterenberg, Absolute continuity of the spectrum of the Schrödinger operator with the potential concentrated on a periodic system of hypersurfaces (Russian, with Russian summary), Algebra i Analiz 13 (2001), no. 5, 197-240; English transl., St. Petersburg Math. J. 13 (2002), no. 5, 859-891. MR1882869 (2002m:35172)

[352] M. A. Shubin, Spectral theory and the index of elliptic operators with almost-periodic coefficients (Russian), Uspekhi Mat. Nauk 34 (1979), no. 2(206), 95-135. MR535710 (81f:35090)

[353] M. A. Shubin, Weak Bloch property and weight estimates for elliptic operators, Séminaire sur les Équations aux Dérivées Partielles, 1989-1990, École Polytech., Palaiseau, 1990, pp. Exp. No. V, 36. With an appendix by Shubin and J. Sjöstrand. MR1073180 (92f:58167)

[354] B. Simon, On the genericity of nonvanishing instability intervals in Hill's equation, Ann. Inst. H. Poincaré Sect. A (N.S.) 24 (1976), no. 1, 91-93. MR0473321 (57 \#12992)

[355] B. Simon, Trace ideals and their applications, 2nd ed. Mathematical Surveys and Monographs, vol. 120, American Mathematical Society, Providence, RI, 2005. MR2154153 (2006f:47086) 
[356] B. Simon, Szego's theorem and its descendants, spectral theory for $l^{2}$ perturbations of orthogonal polynomials, Princeton University Press, Princeton, NJ, 2011.

[357] J. Sjöstrand, Microlocal analysis for the periodic magnetic Schrödinger equation and related questions, Microlocal analysis and applications (Montecatini Terme, 1989), Lecture Notes in Math. vol. 1495, Springer, Berlin, 1991, pp. 237-332, DOI 10.1007/BFb0085125. MR.1178559 (94f:35119)

[358] M. M. Skriganov, Geometric and arithmetic methods in the spectral theory of multidimensional periodic operators (Russian), Trudy Mat. Inst. Steklov. 171 (1985), 122. MR798454 (87h:47110)

[359] M. M. Skriganov, The spectrum band structure of the three-dimensional Schrödinger operator with periodic potential, Invent. Math. 80 (1985), no. 1, 107-121, DOI 10.1007/BF01388550. MR784531 (86i:35107)

[360] M. M. Skriganov and A. V. Sobolev, Asymptotic estimates for spectral bands of periodic Schrödinger operators (Russian, with Russian summary), Algebra i Analiz 17 (2005), no. 1, 276-288, DOI 10.1090/S1061-0022-06-00900-9; English transl., St. Petersburg Math. J. 17 (2006), no. 1, 207-216. MR2140682 (2005m:35205)

[361] V. P. Smyshlyaev and P. Kuchment, Slowing down and transmission of waves in high contrast periodic media via "non-classical" homogenization, unpublished (2007).

[362] A. V. Sobolev, Absolute continuity of the periodic magnetic Schrödinger operator, Invent. Math. 137 (1999), no. 1, 85-112, DOI 10.1007/s002220050324. MR1703339 (2000g:35028)

[363] A. V. Sobolev, High energy asymptotics of the density of states for certain periodic pseudodifferential operators in dimension one, Waves in periodic and random media (South Hadley, MA, 2002), Contemp. Math. vol. 339, Amer. Math. Soc., Providence, RI, 2003, pp. 171-184, DOI 10.1090/conm/339/06105. MR2042537(2005a:35216)

[364] A. V. Sobolev, Integrated density of states for the periodic Schrödinger operator in dimension two, Ann. Henri Poincaré 6 (2005), no. 1, 31-84, DOI 10.1007/s00023-005-0198-8. MR 2119355(2006b:35056)

[365] A. V. Sobolev, Asymptotics of the integrated density of states for periodic elliptic pseudodifferential operators in dimension one, Rev. Mat. Iberoam. 22 (2006), no. 1, 55-92, DOI 10.4171/RMI/449. MR2267313(2007i:35260)

[366] A. V. Sobolev, Recent results on the Bethe-Sommerfeld conjecture, Spectral theory and mathematical physics: a Festschrift in honor of Barry Simon's 60th birthday, Proc. Sympos. Pure Math., vol. 76, Amer. Math. Soc., Providence, RI, 2007, pp. 383-398. MR2310211 (2009d:35245)

[367] A. V. Sobolev, The gauge transform method in the theory of periodic operators, Lectures at the Isaac Newton Institute, Cambridge (January 2015).

[368] A. V. Sobolev and J. Walthoe, Absolute continuity in periodic waveguides, Proc. London Math. Soc. (3) 85 (2002), no. 3, 717-741, DOI 10.1112/S0024611502013631. MR1936818 (2003j:35240)

[369] S. Stigler, Stigler's law of eponymy, Trans. NY Acad. Sci. 39 (1980), no. 1, 147-157.

[370] T. Sunada, Riemannian coverings and isospectral manifolds, Ann. of Math. (2) 121 (1985), no. 1, 169-186, DOI 10.2307/1971195. MR782558 (86h:58141)

[371] T. Sunada, Fundamental groups and Laplacians, Geometry and analysis on manifolds (Katata/Kyoto, 1987), Lecture Notes in Math., vol. 1339, Springer, Berlin, 1988, pp. 248277. MR961485(89i:58151)

[372] T. Sunada, A periodic Schrödinger operator on an abelian cover, J. Fac. Sci. Univ. Tokyo Sect. IA Math. 37 (1990), no. 3, 575-583. MR1080871 (92e:58222)

[373] T. Sunada, Group $C^{*}$-algebras and the spectrum of a periodic Schrödinger operator on a manifold, Canad. J. Math. 44 (1992), no. 1, 180-193, DOI 10.4153/CJM-1992-011-3. MR 1152674 (93c:58223)

[374] T. Sunada, Discrete geometric analysis, Analysis on graphs and its applications, Proc. Sympos. Pure Math. vol. 77, Amer. Math. Soc., Providence, RI, 2008, pp. 51-83, DOI 10.1090/pspum/077/2459864. MR2459864 (2010h:05006)

[375] T. A. Suslina, On the absence of eigenvalues of a periodic matrix Schrödinger operator in a layer, Russ. J. Math. Phys. 8 (2001), no. 4, 463-486. MR1932011(2003k:81057)

[376] T. A. Suslina, Absolute continuity of the spectrum of the periodic Maxwell operator in a layer (Russian, with English and Russian summaries), Zap. Nauchn. Sem. S.-Peterburg. Otdel. Mat. Inst. Steklov. (POMI) 288 (2002), no. Kraev. Zadachi Mat. Fiz. i Smezh. Vopr. 
Teor. Funkts. 32, 232-255, 274, DOI 10.1023/B:JOTH.0000041481.09722.86; English transl., J. Math. Sci. (N. Y.) 123 (2004), no. 6, 4654-4667. MR.1923552 (2003e:35224)

[377] T. A. Suslina and A. A. Kharin, Homogenization with corrector for a periodic elliptic operator near an edge of inner gap, J. Math. Sci. (N. Y.) 159 (2009), no. 2, 264-280, DOI 10.1007/s10958-009-9437-8. Problems in mathematical analysis. No. 41. MR.2544039 (2010h:35025)

[378] T. A. Suslina and A. A. Kharin, Homogenization with corrector for a multidimensional periodic elliptic operator near an edge of an inner gap, J. Math. Sci. (N. Y.) 177 (2011), no. 1, 208-227, DOI 10.1007/s10958-011-0453-0. Problems in mathematical analysis. No. 59. MR $2838992(2012 \mathrm{k}: 35041)$

[379] T. A. Suslina and R. G. Shterenberg, Absolute continuity of the spectrum of the magnetic Schrödinger operator with a metric in a two-dimensional periodic waveguide (Russian, with Russian summary), Algebra i Analiz 14 (2002), no. 2, 159-206; English transl., St. Petersburg Math. J. 14 (2003), no. 2, 305-343. MR.1925885 (2003h:35185)

[380] V. V. Sviridov, On the completeness of quasienergetic states (Russian), Dokl. Akad. Nauk SSSR 274 (1984), no. 6, 1366-1367. MR740452 (85i:35040)

[381] V. V. Sviridov, Some problems of the theory of differential equations with periodic coefficients, Ph.D. thesis, 1986.

[382] L. E. Thomas, Time dependent approach to scattering from impurities in a crystal, Comm. Math. Phys. 33 (1973), 335-343. MR0334766 (48 \#13084)

[383] D. J. Thouless, Wannier functions for magnetic sub-bands, J. Phys. C: Solid State Phys. 17 (1984), L325-7.

[384] M. Tikhomirov and N. Filonov, Absolute continuity of an "even" periodic Schrödinger operator with nonsmooth coefficients (Russian), Algebra i Analiz 16 (2004), no. 3, 201-210, DOI 10.1090/S1061-0022-05-00866-6; English transl., St. Petersburg Math. J. 16 (2005), no. 3, 583-589. MR2083570 (2005f:35056)

[385] E. C. Titchmarsh, Eigenfunction expansions associated with second-order differential equations. Part I, Second Edition, Clarendon Press, Oxford, 1962. MR.0176151 (31 \#426)

[386] P. van Moerbeke, About isospectral deformations of discrete Laplacians, Global analysis (Proc. Biennial Sem. Canad. Math. Congr., Univ. Calgary, Calgary, Alta. 1978), Lecture Notes in Math. vol. 755, Springer, Berlin, 1979, pp. 313-370. MR564908 (81g:58018)

[387] O. A. Veliev, The spectrum of multidimensional periodic operators (Russian), Teor. Funktsiu Funktsional. Anal. i Prilozhen. 49 (1988), 17-34, 123, DOI 10.1007/BF02216095; English transl., J. Soviet Math. 49 (1990), no. 4, 1045-1058. MR963619 (89j:35102)

[388] O. A. Veliev, Perturbation theory for the periodic multidimensional Schrödinger operator and the Bethe-Sommerfeld conjecture, Int. J. Contemp. Math. Sci. 2 (2007), no. 1-4, 19-87. MR2292470 (2009b:47080)

[389] O. Veliev, Multidimensional periodic Schrödinger operator, Springer Tracts in Modern Physics, vol. 263, Springer, Cham, 2015. Perturbation theory and applications. MR 3328527

[390] J. Von Neumann and E. P. Wigner, Über merkwürdige diskrete eigenwerte, Z. Phys. 30 (1929), 465-467.

[391] M. Vorobets, On the Bethe-Sommerfeld conjecture for certain periodic Maxwell operators, J. Math. Anal. Appl. 377 (2011), no. 1, 370-383, DOI 10.1016/j.jmaa.2010.10.067. MR2754836 (2012f:35527)

[392] P. R. Wallace, The band theory of graphite, Phys. Rev. 71 (1947), 622.

[393] A. Waters, Isospectral periodic Torii in dimension 2, Ann. Inst. H. Poincaré Anal. Non Linéaire 32 (2015), no. 6, 1173-1188, DOI 10.1016/j.anihpc.2014.06.001. MR3425258

[394] C. H. Wilcox, Theory of Bloch waves, J. Analyse Math. 33 (1978), 146-167, DOI 10.1007/BF02790171. MR516045 (82b:82068)

[395] W. Woess, Random walks on infinite graphs and groups, Cambridge Tracts in Mathematics, vol. 138, Cambridge University Press, Cambridge, 2000. MR.1743100 (2001k:60006)

[396] K. Yajima, Scattering theory for Schrödinger equations with potentials periodic in time, J. Math. Soc. Japan 29 (1977), no. 4, 729-743. MR0470525 (57 \#10276)

[397] K. Yajima, Large time behaviors of time-periodic quantum systems, Differential equations (Birmingham, Ala. 1983), North-Holland Math. Stud. vol. 92, North-Holland, Amsterdam, 1984, pp. 589-597, DOI 10.1016/S0304-0208(08)73745-9. MR799400(86k:81042) 
[398] K. Yajima, Quantum dynamics of time periodic systems, Phys. A 124 (1984), no. 1-3, 613619, DOI 10.1016/0378-4371(84)90277-2. Mathematical physics, VII (Boulder, Colo., 1983). MR 759208

[399] V. A. Yakubovich and V. M. Starzhinskii, Linear differential equations with periodic coefficients. 1, 2, Halsted Press [John Wiley \& Sons] New York-Toronto, Ont.; Israel Program for Scientific Translations, Jerusalem-London, 1975. Translated from Russian by D. Louvish. MR.0364740 (51 \#994)

[400] A. Yariv, Yong Xu, R. Lee, and A. Scherer, Coupled-resonator optical waveguide: a proposal and analysis, Opt. Lett. 24 (1999), 711-713.

[401] S. T. Yau, Harmonic functions on complete Riemannian manifolds, Comm. Pure Appl. Math. 28 (1975), 201-228. MR0431040 (55 \#4042)

[402] V. I. Yudovich, The linearization method in the stability problem for periodic flows of low viscosity fluids, Proceedings of the VI- th winter school on mathematical programming and related problems, Drogobych 1973: Functional analysis and its applications. (In Russian) (1975), 44-113.

[403] V. I. Yudovich, The linearization method in hydrodynamical stability theory, Translations of Mathematical Monographs, vol. 74, American Mathematical Society, Providence, RI, 1989. Translated from the Russian by J. R. Schulenberger. MR 1003607 (90h:76001)

[404] M. G. Zaŭdenberg, S. G. Krě̆n, P. Kučment, and A. A. Pankov, Banach bundles and linear operators (Russian), Uspehi Mat. Nauk 30 (1975), no. 5(185), 101-157. MR0415661 (54 \#3741)

[405] J. Zak, Magnetic translaion group: II. Irreducible representations, Phys. Rev. (2) 134 (1964), A1602-A1606. MR0177769 (31 \#2031)

[406] Ya. B. Zeldovich, Scattering and emission of a quantum system in a strong electromagnetic wave, Soviet Physics Uspekhi 16 (1973), 427-433.

[407] V. V. Zhikov, Gaps in the spectrum of some elliptic operators in divergent form with periodic coefficients (Russian), Algebra i Analiz 16 (2004), no. 5, 34-58, DOI 10.1090/S1061-002205-00878-2; English transl., St. Petersburg Math. J. 16 (2005), no. 5, 773-790. MR 2106666 (2006a:35053)

[408] J. M. Ziman, Electrons in metals. A short guide to the Fermi surface, Taylor \& Francis, London, 1962.

[409] J. M. Ziman, Principles of the theory of solids, Cambridge University Press, New York, 1964.

[410] N. A. Zimbovskaya, Local geometry of the Fermi surface, and high-frequency phenomena in metals, Springer Verlag, New York, 2001.

Department of Mathematics, Texas A\&M University, College Station, Texas 778433368

E-mail address: kuchment@math.tamu.edu 